\title{
Characterization of Mechanical Properties of Battery Electrode Films from Acoustic Resonance Measurements
}

Kathryn Lanae Dallon

Brigham Young University

Follow this and additional works at: https://scholarsarchive.byu.edu/etd

Part of the Electrical and Computer Engineering Commons

\section{BYU ScholarsArchive Citation}

Dallon, Kathryn Lanae, "Characterization of Mechanical Properties of Battery Electrode Films from Acoustic Resonance Measurements" (2017). Theses and Dissertations. 6620.

https://scholarsarchive.byu.edu/etd/6620

This Thesis is brought to you for free and open access by BYU ScholarsArchive. It has been accepted for inclusion in Theses and Dissertations by an authorized administrator of BYU ScholarsArchive. For more information, please contact scholarsarchive@byu.edu, ellen_amatangelo@byu.edu. 
Characterization of Mechanical Properties of Battery Electrode Films

from Acoustic Resonance Measurements

Kathryn Lanae Dallon

A thesis submitted to the faculty of

Brigham Young University

in partial fulfillment of the requirements for the degree of

Master of Science

Brian A. Mazzeo, Chair

Dean R. Wheeler

Michael D. Rice

\author{
Department of Electrical and Computer Engineering \\ Brigham Young University
}

Copyright @ 2017 Kathryn Lanae Dallon

All Rights Reserved 


\author{
ABSTRACT \\ Characterization of Mechanical Properties of Battery Electrode Films \\ from Acoustic Resonance Measurements \\ Kathryn Lanae Dallon \\ Department of Electrical and Computer Engineering, BYU \\ Master of Science
}

\begin{abstract}
Measurements of the mechanical properties of lithium-ion battery electrode films can be used to quantify and improve manufacturing processes and to predict the mechanical and electrochemical performance of the battery. This thesis demonstrates the use of acoustic resonances to distinguish among commercial-grade battery films with different active electrode materials, thicknesses, and densities. Resonances are excited in a clamped circular area of the film using a pulsed infrared laser or speaker and responses are measured using an electret condenser microphone. A numerical model is used to quantify the sensitivity of resonances to changes in mechanical properties. When the numerical model is compared to simple analytical models for thin plates and membranes, the battery films measured here trend more similarly to the membrane $m$ odel. Resonance measurements are also used to monitor the drying process. Results from a scanning laser Doppler vibrometer verify the modes excited in the films, and a combination of experimental and simulated results is used to estimate the Young's modulus of the battery electrode coating layer.
\end{abstract}

Keywords: battery, acoustics, resonance, material characterization 


\section{ACKNOWLEDGMENTS}

I would like to thank my parents for all of their support and encouragement. Thanks to my

advisor Dr. Brian Mazzeo for all of his help and guidance, and for giving me the opportunity to do research, even as an undergraduate, and to present at conferences. He gave me the chance to work on multiple projects, which ended up being interesting and a great learning experience for me. I also want to thank my fellow students in Dr. Mazzeo's research groups. Thanks to Jing Yao, Michael Wray, and Joseph Cassler for their assistance on this project. 


\section{TABLE OF CONTENTS}

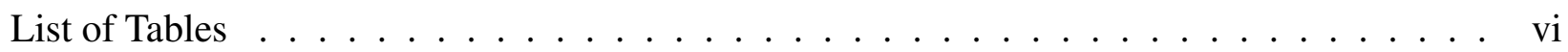

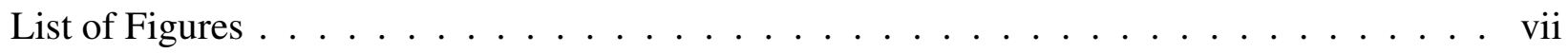

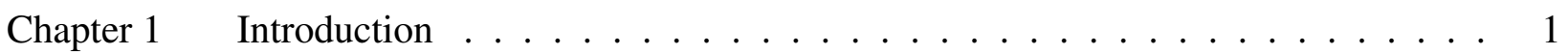

1.1 Problem Statement . . . . . . . . . . . . . . . . . . . . 1

1.2 How Li-Ion Batteries Work . . . . . . . . . . . . . . . . . . . . . 2

1.3 Battery Film Structure . . . . . . . . . . . . . . . . . . . 3

1.4 Impact . . . . . . . . . . . . . . . . . . . . 5

1.5 Previous Acoustic Work . . . . . . . . . . . . . . . . 6

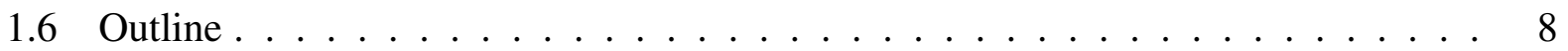

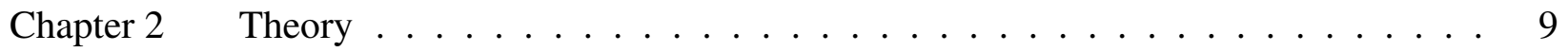

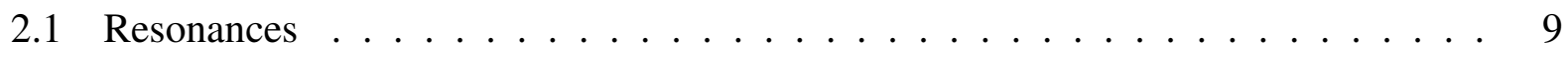

2.2 Thin Plate v. Membrane Theory . . . . . . . . . . . . . . . . . 10

2.3 Mode Shapes . . . . . . . . . . . . . . . . . . . 11

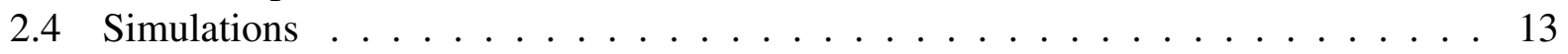

Chapter 3 Experimental Methods . . . . . . . . . . . . . . . 20

3.1 Preliminary Setups . . . . . . . . . . . . . . . . . 20

3.2 Experimental Apparatus . . . . . . . . . . . . . . . . . 24

3.3 Laser . . . . . . . . . . . . . . . . . . . . . . . 28

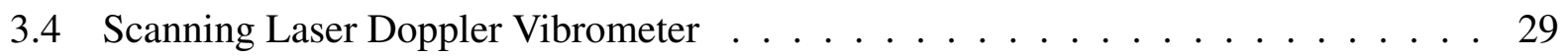

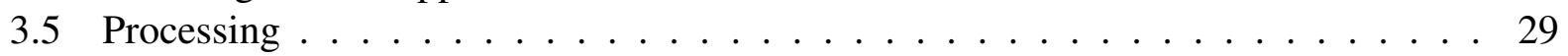

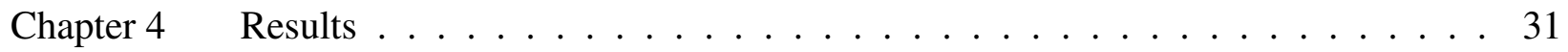

4.1 Battery Films . . . . . . . . . . . . . . . . . . 31

4.2 Scanning Laser Doppler Vibrometer . . . . . . . . . . . . . . . . . 31

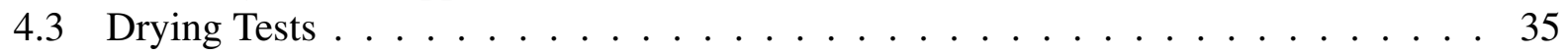

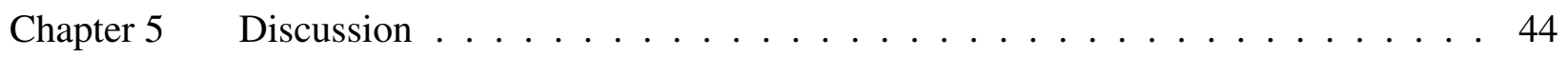

5.1 Thin Plate/Membrane Behavior in Results . . . . . . . . . . . . . . . . . . . 44

5.2 Extracting Young's Modulus . . . . . . . . . . . . . . 51

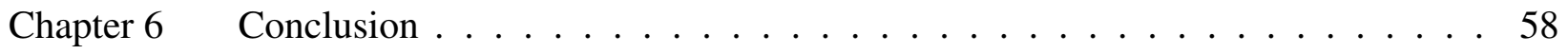

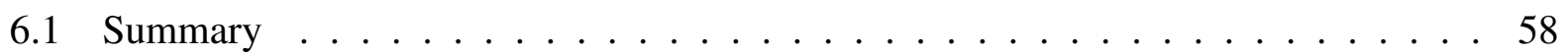

6.2 Future Work . . . . . . . . . . . . . . . . . . . . . . . . . . . . . . . . 68

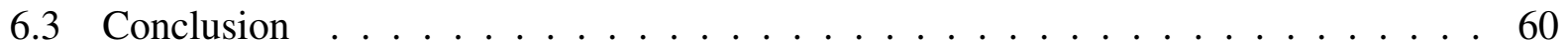

References ............................... 61 
Appendix A Additional Information . . . . . . . . . . . . . . . 66

A.1 MATLAB Code . . . . . . . . . . . . . . . . . . . 66

A.2 Analog Discovery Settings . . . . . . . . . . . . . . . . . 72 


\section{LIST OF TABLES}

2.1 Values of $\alpha_{n s}$ for Thin Plates . . . . . . . . . . . . . . . . . . . 12

2.2 Values of $\alpha_{n s}$ for Membranes . . . . . . . . . . . . . . . . . . 13

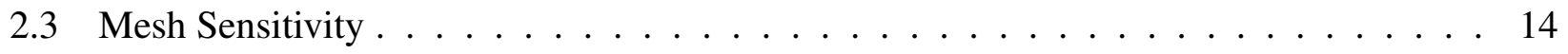

2.4 Model Parameters . . . . . . . . . . . . . . . . . . . . . . 15

2.5 Frequencies for Aluminum Film . . . . . . . . . . . . . . . . . . 16

4.1 Tested Film Properties $\ldots \ldots \ldots \ldots$

4.2 Film Peaks . . . . . . . . . . . . . . . . . . . . 40

5.1 Film Properties . . . . . . . . . . . . . . . . . . . . . 47

5.2 Film Statistics, Steel Clamp . . . . . . . . . . . . . . . . . . . 50

5.3 Film Statistics, Acrylic Clamp . . . . . . . . . . . . . . . . . 51 


\section{LIST OF FIGURES}

1.1 Research Approach . . . . . . . . . . . . . . . . . . . . . . . . 2

1.2 Electrochemical Cell Diagram $\ldots \ldots \ldots \ldots \ldots \ldots \ldots$

1.3 Battery Diagram . . . . . . . . . . . . . . . . . . . 4

1.4 Battery Cathode Film Cross-Section $\ldots \ldots \ldots \ldots$. . . . . . . . . . 5

2.1 Modal Analysis Simulation Mode Shapes _ . . . . . . . . . . . . . . . . . . . 12

2.2 Modal Analysis Simulation Mesh . . . . . . . . . . . . . . . . . . . . . . . . . 13

2.3 Models of Aluminum Film . . . . . . . . . . . . . . . . . . . . . . . . . . 17

2.4 Models of Battery Film with Varying Thickness . . . . . . . . . . . . . . . . 18

2.5 Models of Battery Film with Varying Density . . . . . . . . . . . . . . . . . . . . . . 19

2.6 Models of Battery Film with Varying Young's Modulus . . . . . . . . . . . . . . . . . 19

3.1 Photo of Metal Clamps . . . . . . . . . . . . . . . . . . . . . 21

3.2 Photo of Vacuum Clamps . . . . . . . . . . . . . . . . . . . . 21

3.3 Photo of Clamps . . . . . . . . . . . . . . . . . . . . . . . 22

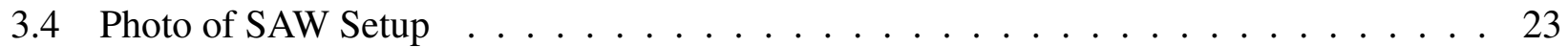

3.5 Photo of Microphones . . . . . . . . . . . . . . . . . . . . . . . . 24

3.6 Illustration of Experimental Apparatus, Laser . . . . . . . . . . . . . . . . . . 25

3.7 Illustration of Experimental Apparatus, Speaker . . . . . . . . . . . . . . . 26

3.8 Photo of Acrylic Clamp . . . . . . . . . . . . . . . . . . . . . . 26

3.9 Photo of Steel Clamp . . . . . . . . . . . . . . . . . . . . . . 27

3.10 Photo of Steel Clamp Setup . . . . . . . . . . . . . . . . . . . . . . . 27

3.11 Photo of IR Laser . . . . . . . . . . . . . . . . . . . . . 28

3.12 Photo of SLDV Setup . . . . . . . . . . . . . . . . . . . . . . . 29

4.1 Photo of Tested Films . . . . . . . . . . . . . . . . . . . . . . . . 32

4.2 Time-Domain Measurements for Battery Films . . . . . . . . . . . . . . . . . 33

4.3 Power Spectral Density for Battery Films, Steel Clamp . . . . . . . . . . . . . . . . . 34

4.4 Power Spectral Density for Battery Films, Acrylic Clamp . . . . . . . . . . . . . . . . . 34

4.5 SLDV Spectrum, Coating 1 . . . . . . . . . . . . . . . . . . . . . 35

4.6 SLDV Map, Coating 1, Peak $1 \ldots \ldots \ldots \ldots \ldots$

4.7 SLDV Map, Coating 1, Peak $2 \ldots \ldots \ldots \ldots$. . . . . . . . . . . . 36

4.8 SLDV Map, Coating 1 , Peak $3 \ldots \ldots \ldots \ldots \ldots$

4.9 SLDV Spectrum, Coating $2 \ldots \ldots \ldots \ldots$

4.10 SLDV Map, Coating $2 \ldots \ldots \ldots \ldots \ldots$

4.11 SLDV Spectrum, Coating $3 \ldots \ldots \ldots$. . . . . . . . . . . . . 38

4.12 SLDV Map, Coating $3 \ldots \ldots \ldots \ldots$

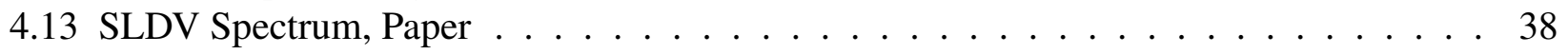

4.14 SLDV Map, Paper . . . . . . . . . . . . . . . . . . . . . . . . . . . . . 39

4.15 SLDV Spectrum, Acrylic Clamp . . . . . . . . . . . . . . . . . . . . . 39

4.16 SLDV Map, Acrylic Clamp . . . . . . . . . . . . . . . . . . . . . . . 40

4.17 Frequency Shift, Dried Paint, Speaker Excitation . . . . . . . . . . . . . . . . 40

4.18 Frequency Shift, Drying Paint, Speaker Excitation . . . . . . . . . . . . . . . . 41 
4.19 Frequency Shift, Drying Paint, Laser Excitation . . . . . . . . . . . . . . . . 41

4.20 Frequency Peaks, Drying Paint, Laser Excitation . . . . . . . . . . . . . . . . . 42

4.21 Frequency Shift, Drying Paint (Undiluted), Laser Excitation ～. . . . . . . . . . . . 43

4.22 Frequency Peaks, Drying Paint (Undiluted), Laser Excitation . . . . . . . . . . . . . 43

5.1 Simulation of Battery Cathode, Varying Mass . . . . . . . . . . . . . . . . . . 45

5.2 Simulation of Battery Cathode, Varying Radius . . . . . . . . . . . . . . . . . 48

5.3 Simulation of Battery Cathode, Coating 1, Varying Radius . . . . . . . . . . . . . . . . 49

5.4 Simulation of Battery Cathode, Coating $1 \ldots \ldots \ldots$

5.5 Simulation of Battery Cathode, Coating $2 \ldots \ldots \ldots \ldots$

5.6 Simulation of Battery Cathode, Coating $3 \ldots \ldots$. . . . . . . . . . 55

5.7 Simulation of Battery Cathode, Coating 1 (YM $344 \mathrm{MPa}$ ), with Varying Al Elasticity . 56

5.8 Simulation of Battery Cathode, Coating 1 (YM $87 \mathrm{MPa}$ ), with Varying Al Elasticity . . 56

5.9 Simulation of Battery Cathode, Coating 2, with Varying Al Elasticity . . . . . . . . . 57

5.10 Simulation of Battery Cathode, Coating 3, with Varying Al Elasticity . . . . . . . . . . 57

A.1 Electret Microphone Circuit . . . . . . . . . . . . . . . 73 


\section{CHAPTER 1. INTRODUCTION}

Batteries are ubiquitous in the world today. Everything from a car or satellite to a phone or wearable device is powered by batteries. Many of these batteries are lithium-ion (Li-ion) batteries. Due to their large energy density ( $\geq 200 \mathrm{Wh} / \mathrm{kg}$ [1]) and ability to charge and discharge at high power, Li-ion batteries are ideal for many applications. The demand for batteries keeps growing, particularly in applications for electric vehicles and renewable energy. In 2016, over 750,000 electric cars were sold across the globe. Many more batteries will need to be produced in the future, as multiple countries have set "deployment targets" for electric vehicles in order to promote clean energy, adding up to a worldwide goal of about 13 million electric cars by 2020 [2]. Even a small improvement in battery capacity can have a large impact, as billions of Li-ion batteries are produced each year [3], and that number will continue to rise. With such huge quantities, it will become increasingly important to have more powerful and reliable batteries.

One important area of research to improve Li-ion batteries is to understand and measure heterogeneity in battery electrode properties [4], [5], particularly before the electrode is incorporated into a full cell. Non-uniformity across an electrode in properties like material distribution and electrical conductivity can possibly lead to worsening defects over time and affect the battery quality [6], [7]. If problems with the electrodes can be identified and addressed during manufacturing, efficiency can be increased and more reliable batteries produced.

\subsection{Problem Statement}

This research investigates acoustic measurements as a non-destructive method to characterize the mechanical properties of battery films. Specifically, I report on my results in distinguishing among films with different mechanical properties using acoustic resonances. Materials with a plate-like geometry vibrate at different frequencies depending on area, density, thickness, and rigidity, so changes in these parameters cause a change in resonance frequency that can be 
measured. Using known mechanical properties for the battery materials, I created a parameterized numerical model using ANSYS simulation software. Additionally, I compare the results from this numerical model to the simple analytical models that describe thin plate and membrane vibrations. I then use a combination of experimental acoustic measurements and the simulation results to estimate the unknown parameters of battery electrodes and validate the numerical model. This approach is illustrated in Fig. 1.1.

This research has been developed with the long-term goal of creating an acoustic probe to detect small changes in a battery film's local material properties, in particular, density and thickness. The proposed acoustic probe can be used in conjunction with a conductivity probe [8] to provide better understanding of the electrode properties. This combination of material parameters can then inform modeling work as well as process improvements.

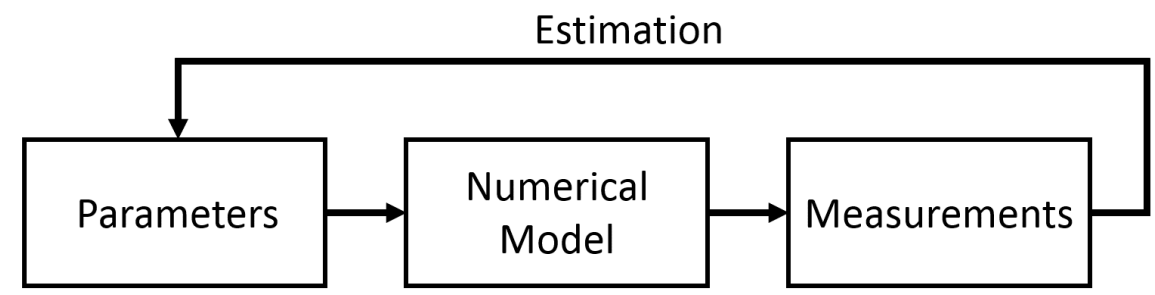

Figure 1.1: Diagram of the approach taken for this research. Parameters for battery materials and geometry of electrode films are used to create a numerical model in ANSYS software. Acoustic measurements taken on battery films are then used to estimate unknown battery parameters and validate the model.

\subsection{How Li-Ion Batteries Work}

The basic element of a battery is the electrochemical cell, which can be connected with others in parallel or series configurations within the battery casing. An electrochemical cell is composed of a separator and an electrolyte solution between two electrodes (the cathode and anode). As its name indicates, the separator keeps the electrodes spaced evenly apart and blocks electron flow but allows ions to move between the two electrodes. This structure is depicted in Fig. 1.2. When the cell is discharged, as shown in the figure, ions move through the separator inside the cell to promote electron flow. This electronic current flows through the metal current 


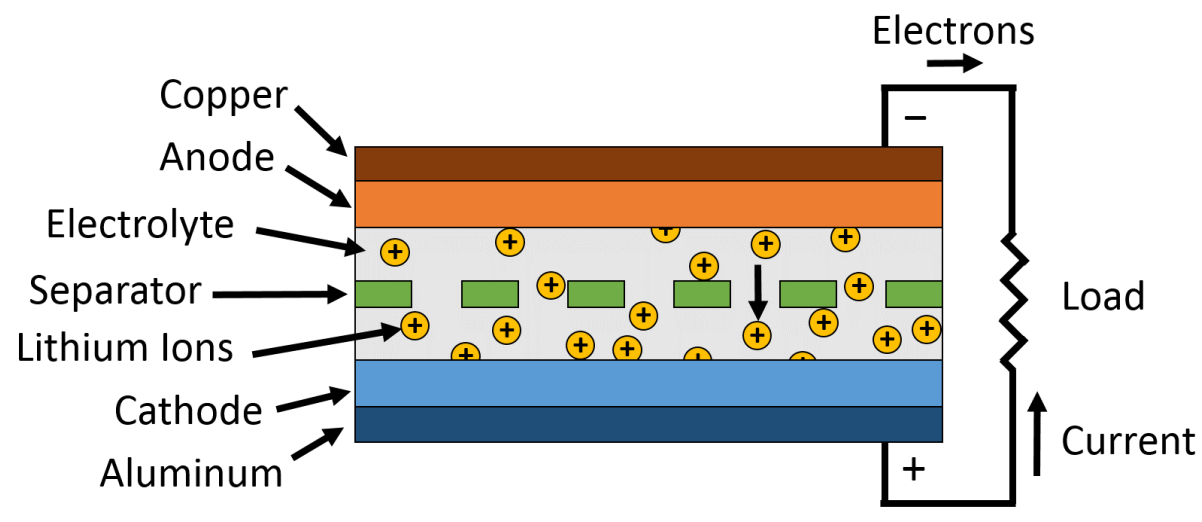

Figure 1.2: Diagram of an electrochemical cell. The internal movement of ions (in this case, lithium ions) from one electrode to the other causes electrons to flow between electrodes through the current collectors and external circuit [10]. The direction of ion and electron flow shown here corresponds to the discharging state of the cell.

collectors and outside the cell from the cathode to the anode, providing power across the connected load. The direction of flow for both ions and electrons is reversed during charging [9], [10].

Li-ion battery electrodes are generally a thin, metal current collector coated on both sides with anode or cathode material. In most commercial battery manufacturing processes, the battery coating materials are mixed and applied to the metal current collector as a wet slurry, which then has to be dried. The electrodes are then calendered, or pressed, to a specific thickness, which also increases the density and changes the porosity of the electrode. When a cylindrical Li-ion battery is formed, the electrode films are layered with the separator between them (anode current collector anode - separator - cathode - cathode current collector - cathode - separator - anode - anode current collector) and then rolled up to fit into the battery case, as illustrated in Fig. 1.3. The electrolyte solution is later added to the cell [11].

\subsection{Battery Film Structure}

Single-sided Li-ion battery electrode films are essentially two-layer structures. The metal current collector, generally made of aluminum for cathodes and copper for anodes, is one layer. On top of this layer is a coating layer consisting of the cathode or anode material. In cathodes, this material is a mixture of large $(\sim 1-20 \mu \mathrm{m})$ active material particles, carbon additive for increased 


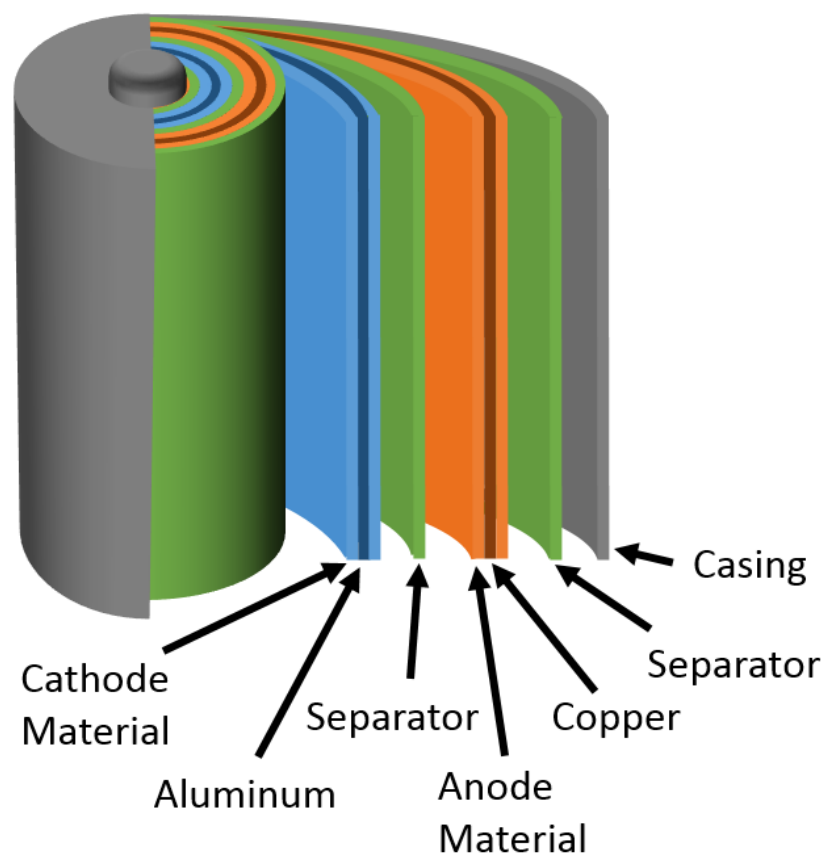

Figure 1.3: Diagram of formed cylindrical battery. The anode- and cathode-coated current collectors are layered with the separator between them and rolled up inside the battery case [9].

electronic conductivity, polymeric binder, and pores (both large pores and nanopores). An example of this layered structure and its constituent materials is shown in Fig. 1.4. In this thesis, the top layer will be called the coating, while "film" refers to the entire two-layer structure of the electrode.

The homogeneity of the coating layer in battery films is important, both in properties like thickness and material distribution. Variations in the battery coating properties can create "hot" or "cold" areas of conductivity across the electrode, which can possibly lead to increasing defects over time as the battery is used. Non-uniformity in properties like current distribution can even cause premature battery failure [11], and differences between battery cells in a pack will worsen over time and can lead to a shorter battery pack lifetime [12]. Measuring the mechanical properties of lithium-ion battery films, such as thickness and elasticity, could be important for predicting and improving homogeneity of the films and subsequent performance of the battery [4], as pristine commercial battery electrodes can exhibit significant variation in structure across a millimeter scale [8]. Variations in macroscopic properties, like thickness, elastic modulus, and tortuosity, 


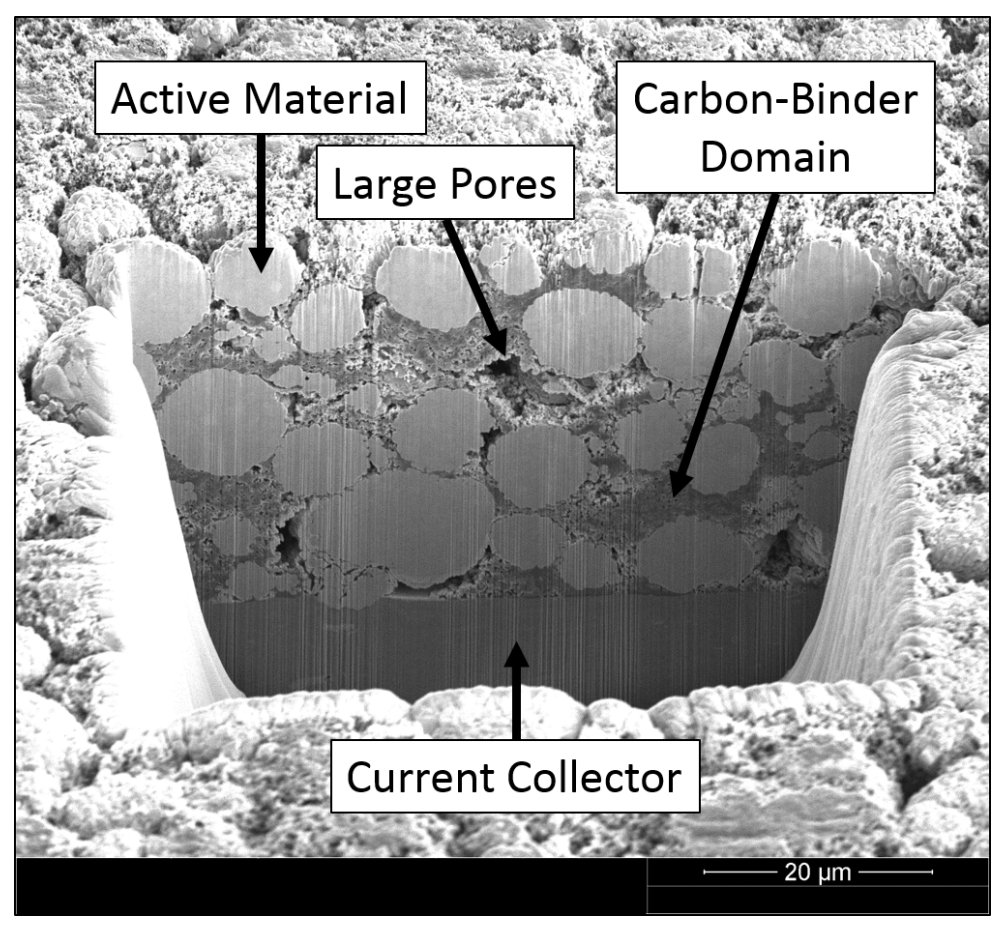

Figure 1.4: Scanning electron microscope micrograph of a Li-ion cathode battery film (Toda 523) in cross-section, cut using a focused ion beam. The bottom layer is the aluminum current collector. The top layer is the cathode coating and is made up of NCM active material (lighter circles), carbon conductivity additive and polymeric binder (nanoporous gray filler between active material), and empty pores (dark spaces between active material and binder).

can indicate differences in microscopic properties, like material distribution, that cause inhomogeneities in electrical and ionic conductivity [5], [13] and can influence the battery capacity and lifetime [6], [7].

\subsection{Impact}

Correlating heterogeneity in electrode properties with battery performance would provide an important area for manufacturers and researchers to work in to improve homogeneity and therefore battery performance. It would be advantageous if problems with film heterogeneity could be identified and addressed through accurate, non-destructive inspection of the electrode as it is being manufactured. There are several techniques currently used to measure the mechanical characteristics of films, including nanoindentation [13], [14] and Brillouin scattering [15], [16]. However, they have not extensively been used for analyzing battery electrodes. Additionally, many of the methods used in manufacturing to measure mechanical properties are destructive in some way. 
For example, density can be easily calculated using weight and volume measurements, but finding local density in this manner requires the film to be cut into small pieces to be weighed. Other measurements require the current collector to be removed from the film. Due to the destructive nature of many testing methods, current quality control often involves taking a piece of the sheet, testing it, and assuming that it is representative of the whole. Ideally, we want to have a method for measurement that doesn't require destruction of the film so that pristine films can be characterized with multiple measurements across the electrode sheet and then used in a battery. This work moves toward the goal of a non-destructive quality control test.

\subsection{Previous Acoustic Work}

Using acoustics for non-destructive measurements has been applied to many areas using many different methods. Research has been done using a variety of acoustic techniques as a method for damage detection in non-battery structures, including concrete bridges [17], storage containers for spent nuclear fuel [18], and aircraft structures [19].

Another area of research is using acoustics to determine characteristics of thin films using surface acoustic waves and Lamb waves. As their name suggests, surface acoustic waves (SAWs) propagate mainly along the surface of a material, making them ideal for looking at thin films on a substrate. The depth of wave penetration depends on the frequency of the wave. At different frequencies, the wave interacts with the substrate to varying degrees, causing a dispersion in wave velocity. This velocity dispersion can be used to find the elastic properties and thickness of the thin film [20]. This technique has been used to find one or two mechanical properties of various thin films less than $1 \mu \mathrm{m}$ thick [21], [22], the Young's modulus of films as thin as $100 \mathrm{~nm}$ [20], the Young's modulus and porosity of 500-nm-thick films [23], the elastic properties of ultrathin (less than $40 \mathrm{~nm}$ thick) films [24], changes in a steel surface due to radiation damage [25], and changes in the Young's modulus of films of shape memory alloys at different temperatures [26]. It has also been compared to nanoindentation for finding the elastic modulus of thin films with a high surface roughness [27] and nanostructured films with surface roughness [28], where the SAW method gave results similar to or better than nanoindentation.

Lamb waves are guided waves in thin plates and can propagate long distances with little attenuation [19]. For isotropic plates, Lamb waves can be symmetric or antisymmetric, depending on 
the direction of the displacement. Symmetric, or compression, waves mainly have motion within the plane of the plate, and antisymmetric, or flexural, waves have significant motion normal to the plate surface [19], [29]. Like SAWs, Lamb waves are dispersive, allowing for the determination of elastic properties and thickness of the plate [29]. Because of this, Lamb waves have been used to find the flexural modulus of thin, metallic, free-standing films [30]; thickness and bulk velocities of thin plates [29]; and changes in surface roughness in aluminum structures [19].

One specific research area of interest relating to Lamb waves is using the zero-groupvelocity $(\mathrm{ZGV})$ Lamb wave modes to find information about a material. These modes occur where the slope of the dispersion curve of the Lamb waves is zero, and therefore the group velocity is also zero. Because the group velocity is related to energy movement, when the group velocity is zero, energy does not propagate away from the source, resulting in a sharp plate resonance [31], [32]. By performing an ultrasonic scan near the $\mathrm{ZGV}$ resonance, Holland, et al. were able to detect small thickness changes and simulated flaws in plates [31]. Several studies used shifts in ZGV resonances to find small thickness variations in plates [33], [34]. Measuring multiple ZGV frequencies enables the inversion of plate characteristics such as thickness or elastic properties [35]. However, the frequency of vibrations for these $\mathrm{ZGV}$ resonances is rather high. For most of the plates studied in the papers above, the resonances were in the megahertz range, requiring detection methods with a high sampling rate. Plates thicker than $1 \mathrm{~mm}$ may have resonances in the kilohertz range [31], but thinner plates or films will have resonances at much higher frequencies.

The frequency of vibration of materials has been used in the past to find properties like Young's modulus and stress. In one study, Fartash used the shifts in resonance frequency of metal films (less than $20 \mu \mathrm{m}$ thick) to find the elasticity of the films [36]. Biswas, et al. calculated the stress of a membrane by using a combination of the frequencies at which it vibrated and the known properties of the membrane [37]. Malhaire, et al. compared stress and Young's modulus measurements from the frequencies of vibration of thin metallic membranes with measurements from the bulge testing method and found that for sub-micron thick films, both measurement techniques had good agreement [38]. In a recent paper, Gamboa, et al. used a cantilever beam configuration to find the natural frequencies of thin films on a substrate. By measuring the shifts in frequency as additional sub-micron layers were added, the elastic modulus of each of the layers can be found [39]. 
Other studies have used similar acoustic methods to examine non-film structures. Costley, et al. did a study looking at the shift in the resonance frequencies of storage lids to determine changes in the internal pressure of the sealed storage drums [40]. Hsieh, et al. used acoustic time-of-flight measurements to find the state of charge and health in packaged battery cells. In this study, they used transducers to apply a pulse and then listen to the reflected and transmitted signals through a battery. They were able to relate the changes in the speed of sound to the charge and health of the battery because of the density and modulus changes of the internal materials as the battery was charged and discharged [41].

While most of these studies do not specifically involve batteries, many of the principles can be applied to studying battery electrodes. In particular, we draw on the past work that looks at membranes and material vibrations. The theory behind the research in this thesis has to do with the vibration of thin plates and membranes, which is discussed in detail in Chapter 2 . We extend the use of some of the same acoustic principles as these previous studies to examine the mechanical properties of battery electrode films.

\subsection{Outline}

In this thesis, I describe my work in using acoustic resonance measurements to differentiate between battery films with different mechanical properties. As illustrated in Fig. 1.1, I have used known parameters to create a numerical model and compared this to acoustic measurements of battery films in order to estimate the unknown parameters of the films and validate the numerical model. Chapter 2 gives an overview of the theory behind these acoustic measurements and how changing the mechanical properties is expected to change the resonances. In Chapter 3, the various setups used to take the measurements are described, along with the processing method for the data. The results from the measurements are given in Chapter 4 and discussed in Chapter 5. A summary, my suggestions for future work, and a conclusion comprise Chapter 6. 


\section{CHAPTER 2. THEORY}

Most people have observed resonance in some way, such as in changing the amount of water in a glass to see how the pitch changes when tapped, or striking a drum head at various positions to get different sounds. The frequency emitted by either of these sources changes based on the properties of the system-for the glass, the volume of water, and for the drum, the tension on the drum head and location of the strike. This chapter discusses how, in a similar manner, the properties of battery films determine the frequencies at which the films vibrate.

\subsection{Resonances}

When looking at how films and other thin structures vibrate, the natural frequencies are the frequencies corresponding to the solutions of the two-dimensional wave equation

$$
\frac{\partial^{2} z}{\partial x^{2}}+\frac{\partial^{2} z}{\partial y^{2}}=\frac{1}{c^{2}} \frac{\partial^{2} z}{\partial t^{2}}
$$

where $z(x, y, t)$ is the $z$-displacement at time $t$ and location $(x, y)$, in cartesian coordinates, and $c$ is wave speed in the material [42]. The solutions will change based on the given boundary conditions of the system. These solutions are the frequencies at which the system will freely vibrate. If the vibration is forced, resonance occurs when the frequency of the driven force matches the natural frequency of the system [43]. These resonance frequencies are those for which the maximum vibration amplitude occurs for a given excitation [42]. In this thesis, the terms "resonance frequency" and "natural frequency" are used interchangeably. The resonance frequency of a vibrating object depends on several factors, including the shape, size, and material properties.

When solving for how materials vibrate, thin plates and membranes have similar theory, but membrane theory is somewhat simplified. The only restoring force on a membrane comes from the tension, while a thin plate has a restoring force from its stiffness [42]. These two types of 
systems can be distinguished by the ratio $a / h$, where $a$ is the characteristic length of the material and $h$ is the thickness. Thin plates typically have an $a / h$ ratio ranging from about 8 to 80 , while ratios of $a / h>80$ are associated with membranes [44]. In our case, we are generally looking at a characteristic dimension of $a \approx 1 \mathrm{~cm}$. The thickness of many battery electrode films range from about 20-60 $\mu \mathrm{m}$, resulting in an $a / h$ ratio ranging from $\sim 150-500$. However, even though the $a / h$ ratio indicates our films are in the membrane region, they don't fully qualify as membranes, because a true membrane doesn't have any stiffness. They also don't act fully like a thin plate, due to the ability to put them under significant tension. However, if the tension applied to a film is large enough, the membrane properties dominate and we can approximate it as a membrane with some perturbations due to the rigidity [45]. Although our battery films aren't fully described by either thin plate or membrane theory, particularly because these simple theories are for a single layer made of one material and the battery films have two layers, it can be useful to look at the resonance equations for both theories to give us some intuition as to how we can expect the films to behave.

\subsection{Thin Plate v. Membrane Theory}

The fundamental frequency of a circular thin plate with a clamped boundary is given by

$$
f_{01}=\frac{\alpha_{01}}{2 \pi a^{2}} \sqrt{\frac{D}{\rho h}}
$$

where $\alpha_{01}=10.21, a$ is the radius of the plate, $\rho$ is the density, $h$ is the thickness, and

$$
D=\frac{E h^{3}}{12\left(1-v^{2}\right)}
$$

is the flexural rigidity, with $E$ as the Young's modulus and $v$ as the Poisson's ratio [43]. It is noted that $f_{01}$ will linearly increase with $h$.

The fundamental frequency of a circular membrane is given by

$$
f_{01}=\frac{\alpha_{01}}{2 \pi a} \sqrt{\frac{T}{\rho h}}
$$


where $\alpha_{01}=2.404, a$ is the radius of the membrane, $T$ is the tension, $\rho$ is the density, and $h$ is the thickness [43], [46], [47]. It is noted that $f_{01}$ decreases with increasing $h$.

Equations (2.2) and (2.4) show how the mechanical properties affect the resonance frequencies of a material. Both are dependent on density and thickness, as well as the radius of the vibrating area. Through the rigidity shown in Eq. (2.3), we see that the thin plate theory also relies on the Young's modulus of the material.

Though not perfect models for the battery electrodes, these equations give an approximate prediction for how the resonance frequency changes with thickness, stiffness, tension, and density. Notably, a parameter such as thickness can affect the resonance frequency in opposite ways depending on the true underlying physics which are approximated by these models.

\subsection{Mode Shapes}

Equations (2.2) and (2.4) give expressions for finding the fundamental, or lowest, natural frequency $f_{01}$, but there can be many natural frequencies for a system. A normal mode is an eigenfunction that gives a solution to the wave equation with the given boundary conditions for the situation [42]. Each of these solutions corresponds to a natural frequency. Different mode shapes may have different combinations of nodes. Nodes occur where the displacement of the film is always zero. For a circular geometry, normal nodes can occur in a circular shape (centered with the center of the film) or a line along the diameter of the film [42], [43]. Some mode shapes occur at the same natural frequency. For these degenerate modes, the details of the excitation will determine which mode is excited [42].

When calculating resonance frequencies other than the fundamental frequency, the value of $\alpha_{n s}$ in Eqs. (2.2) and (2.4) must change based on the number of nodes present at the desired frequency $f_{n s}$. The number of nodal diameters is denoted by $n$ and the number of nodal circles, including the fixed boundary, by $s$. Both types of node can be seen in Fig. 2.1, which shows the first six mode shapes for a circular disc with a fixed boundary. The expressions in Eqs. (2.2) and (2.4) used $\alpha_{01}$ in order to find the fundamental frequency $f_{01}$. Values for $\alpha_{n s}$ for some of the first few modes for a circular thin plate with a fixed boundary are given in Table 2.1, and the values for a circular membrane are given in Table 2.2. 


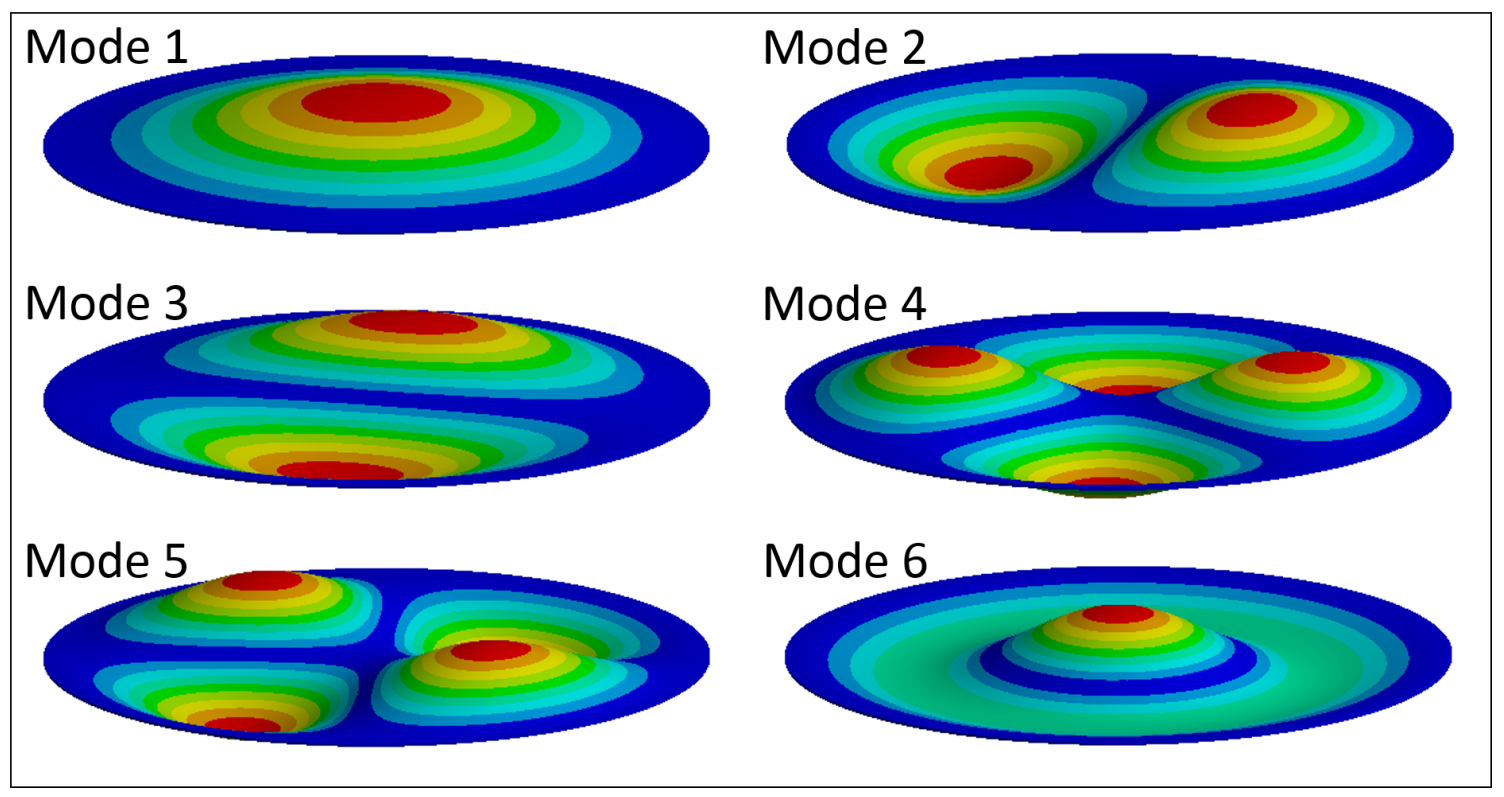

Figure 2.1: ANSYS modal analysis simulation showing the first six mode shapes for a circular area with a fixed boundary. Each mode shape corresponds to a resonance frequency and a value of $\alpha_{n s}$, as seen in Tables 2.1 and 2.2. Mode 1 corresponds to $\alpha_{01}$, modes 2 and 3 correspond to $\alpha_{11}$, modes 4 and 5 correspond to $\alpha_{21}$, and mode 6 corresponds to $\alpha_{02}$, due to the number of nodal diameters $(n)$ and nodal circles $(s)$ present in the mode shape. Modes 2 and 3 and modes 4 and 5 have the same nodal patterns (one and two nodal diameters, respectively) but with different orientations, and they occur at the same or nearly the same frequencies.

The different modes shown in Fig. 2.1 correspond to different values for $\alpha_{n s}$, as given in Tables 2.1 and 2.2. The first mode corresponds to $\alpha_{01}$, the second and third mode shapes to $\alpha_{11}$, the fourth and fifth modes to $\alpha_{21}$, and the sixth mode to $\alpha_{02}$.

Table 2.1: Values for $\alpha_{n s}$ in Eq. (2.2) for a circular plate fixed at the boundary, based on the number of nodal diameters $n$ and the number of nodal circles $s$ (including the boundary) [43], [48].

\begin{tabular}{|c|c|c|c|}
\hline & 0 & 1 & 2 \\
\hline 1 & 10.21 & 21.26 & 34.88 \\
\hline 2 & 39.77 & 60.82 & 84.58 \\
\hline 3 & 89.10 & 120.08 & 153.81 \\
\hline
\end{tabular}


Table 2.2: Values for $\alpha_{n s}$ in Eq. (2.4) for a circular membrane fixed at the boundary, based on the number of nodal diameters $n$ and the number of nodal circles $s$ (including the boundary) [43].

\begin{tabular}{|c|c|c|c|}
\hline & 0 & 1 & 2 \\
\hline 1 & 2.404 & 3.832 & 5.135 \\
\hline 2 & 5.520 & 7.016 & 8.417 \\
\hline 3 & 8.654 & 10.173 & 11.620 \\
\hline
\end{tabular}

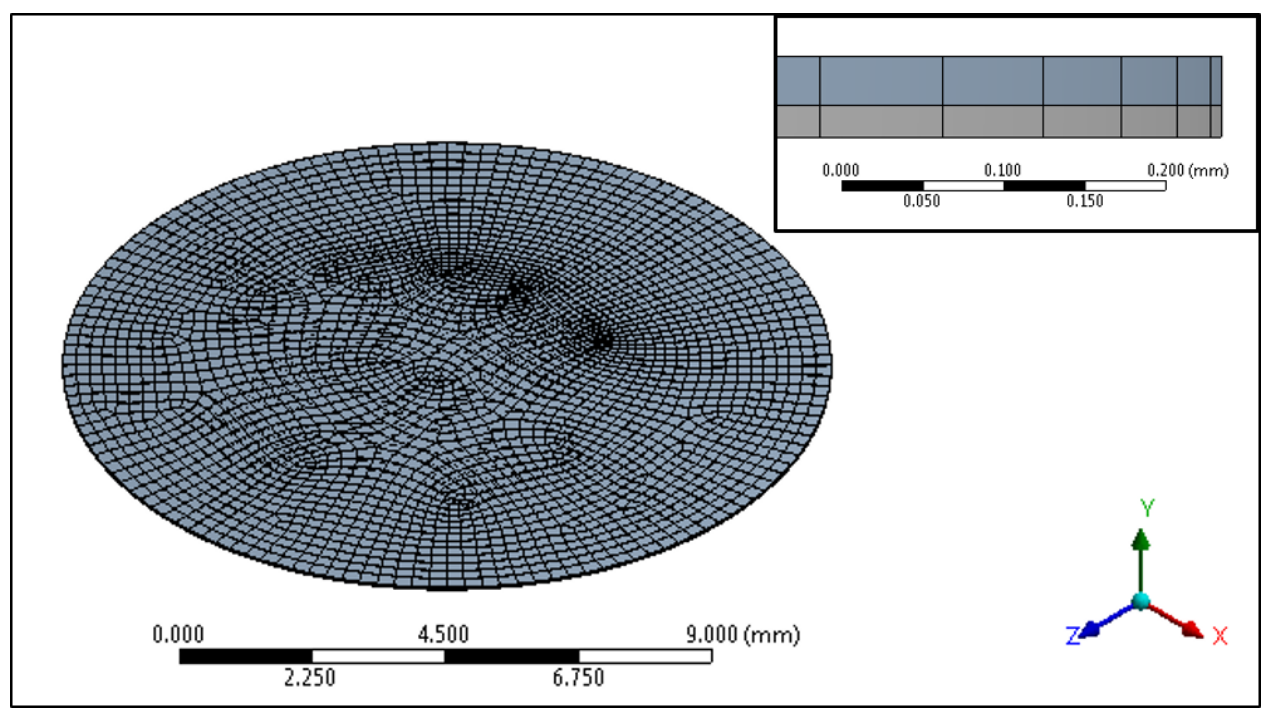

Figure 2.2: ANSYS modal analysis simulation of two-layer battery film with a radius of $0.65 \mathrm{~cm}$, minimum mesh size of $0.01 \mathrm{~mm}$, and maximum mesh size of $0.3 \mathrm{~mm}$. The circumference of the circle is a fixed boundary. The inset shows the side view of the simulation geometry, with the two layers visible (aluminum current collector and battery coating).

\subsection{Simulations}

In order to gain further insight into how we expect the films to resonate, and because simple analytical models are not available for more complicated structures, we created a numerical model using the modal analysis in ANSYS 15.0 (ANSYS, Inc.) simulation software. For all of the simulations, the circumference of the circular geometry (both layers, if applicable) is a fixed edge. An example of the simulation geometry with the meshing visible is shown in Fig. 2.2. Simulations were done for a mesh-sensitivity analysis, as well as for comparison to the analytical models to see how changing thickness, density, and Young's modulus of the battery films would change the resonance frequencies. 
When doing finite element simulations, it is important to do a mesh sensitivity analysis to make sure the size of the elements in the model isn't changing the results. The mesh size used here was chosen because of a combination of short run time and accuracy. While the simulations with more elements can be more accurate, the run time was significantly longer. Simulation results with several different mesh sizes are given in Table 2.3. Mesh 2 (minimum element size $0.01 \mathrm{~mm}$ and maximum size $0.3 \mathrm{~mm}$ ) was the size used for the results in this thesis. Looking at the frequency difference between mesh 1 and 3, it is clear that too coarse of a mesh can have a large impact on the accuracy of the results. Since the difference between mesh 2 and 3 is only $0.4 \%$, the accuracy is reasonable, although a finer mesh (and longer run time) would possibly provide even higher resolution.

Table 2.3: Simulation results for aluminum film with properties shown in Table 2.4, run with various mesh sizes. The frequencies (in $\mathrm{Hz}$ ) shown here indicate that the size of the elements in the analysis can have a large effect on the results.

\begin{tabular}{ccccc}
\hline \hline \multirow{2}{*}{ Mode } & $\begin{array}{c}\text { Mesh 1 } \\
\text { Min: 0.01 mm } \\
\text { Max: 5.0 mm }\end{array}$ & $\begin{array}{c}\text { Mesh 2 } \\
\text { Min: 0.01 mm } \\
\text { Max: 0.3 mm }\end{array}$ & $\begin{array}{c}\text { Mesh 3 } \\
\text { Max: 0.01 } 0.075 \mathrm{~mm}\end{array}$ & $\begin{array}{c}\text { Change } \\
\text { Between } \\
\text { Mesh 2, 3 }\end{array}$ \\
\hline 1 & 856.00 & 829.97 & 826.24 & $0.451 \%$ \\
2 & 1775.8 & 1727.0 & 1719.4 & $0.442 \%$ \\
4 & 2918.9 & 2833.6 & 2820.5 & $0.465 \%$ \\
6 & 3310.4 & 3231.8 & 3216.3 & $0.482 \%$ \\
\hline Elements & 872 & 5470 & 66183 & \\
Run Time & $<10 \mathrm{sec}$ & $\sim 2 \mathrm{~min}$ & $>2$ hours & \\
\hline \hline
\end{tabular}

Using ANSYS, we found the frequencies of the first six mode shapes, which correspond to the lowest four $\alpha_{n s}$ values in Tables 2.1 and 2.2. As mentioned earlier, some mode shapes have degenerate modes that occur at the same (in an ideal case) or similar (when anisotropies are present) frequencies [36]. As seen in Fig. 2.1, for vibration of a circular area with a fixed boundary, this happens with modes 2 and 3 and modes 4 and 5, so we have left the duplicate resonances of modes 3 and 5 out of the subsequent graphs.

The simulation geometry is a disk of radius $0.65 \mathrm{~cm}$ with either one layer (aluminum only) or two layers (aluminum current collector and battery coating) with the material properties listed 
in Table 2.4. The values for the coating properties were taken from a paper by Forouzan et al. [49], other than the Poisson's ratio, which was assumed to be the common value of 0.3 [42].

Table 2.4: Material parameters for aluminum [50] and the battery coating [49] used in the numerical and analytical models.

\begin{tabular}{lcccc}
\hline \hline Material & $h(\mu \mathrm{m})$ & $\rho\left(\mathrm{g} / \mathrm{cm}^{3}\right)$ & $E(\mathrm{GPa})$ & $v$ \\
\hline Aluminum & 20 & 2.70 & 70 & 0.334 \\
Coating & 30 & 2.029 & 0.01132 & 0.3 \\
\hline \hline
\end{tabular}

In order to better understand the vibration behavior of the battery films, we compare the numerical model given by the simulations to the analytical models given by Eqs. (2.2) and (2.4). The numerical model did not involve any pre-tension on the film, so we do not have an applied tension as required for the membrane model. The tension was reverse-calculated using Eq. (2.4) and the resonance frequency from the numerical model result for the first mode and the lowest varied parameter value (which is the reason the membrane model is identical to the simulation for the first point of the first mode). Since the analytical models are both for a single-layer structure, composite parameters were calculated for the battery film using the weighted averages in the rule of mixtures [51]. The total thickness, $h_{t}$, was calculated using

$$
h_{t}=h_{c}+h_{m},
$$

where $h_{c}$ is the coating thickness and $h_{m}$ is the current collector (metal) thickness. The composite density, $\rho_{t}$, was calculated using the rule of mixtures

$$
\rho_{t}=\frac{\rho_{c} h_{c}+\rho_{m} h_{m}}{h_{t}}
$$

where $\rho_{c}$ and $\rho_{m}$ are the densities of the coating and current collector, respectively. Calculating the composite Poisson's ratio is done in the same way as the composite density, but substituting Poisson's ratio $v$ for density in the equation

$$
v_{t}=\frac{v_{c} h_{c}+v_{m} h_{m}}{h_{t}}
$$


The composite Young's modulus, $E_{t}$, was also calculated using the rule of mixtures, but this takes an inverse form for elasticity.

$$
E_{t}^{-1}=\frac{E_{c}^{-1} h_{c}+E_{m}^{-1} h_{m}}{h_{t}},
$$

where $E_{c}$ and $E_{m}$ are the Young's moduli of the coating and current collector. This equation gives the series, or transverse, effective Young's modulus, which may not be a completely accurate estimation, as the motion of the film is not strictly confined to one direction, but it is essentially a lower limit on the composite value. Using an equation with the same form as Eq. (2.6) would give the parallel, or longitudinal, effective modulus, which is the upper limit [51].

Simulations of a single layer of aluminum foil (without any battery coating) have more characteristics of a thin plate; in particular, that the resonance frequency increases with thickness through the rigidity. This is seen in Fig. 2.3, where the simulated modes (shown by the solid line) correspond very well with the thin plate model (shown by the dashed line, which is barely visible under the solid line), but do not follow the trend of the membrane model (shown by the dotted line). Because the lines are difficult to distinguish, some of the frequency values for the numerical and thin plate model are also listed in Table 2.5, where it is clear that many of the values are very close. As the aluminum film is a single-layer structure, it makes sense that it would be accurately described by one of the simple models, and that the numerical and analytical models would have good agreement.

Table 2.5: Frequencies (in $\mathrm{Hz}$ ) for the first four unique mode shapes for an aluminum film of varying thickness. These are the values given by the simulation and thin plate theory plotted in Fig. 2.3.

\begin{tabular}{cccccccc}
\hline \hline \multirow{2}{*}{ Mode } & Model & $10 \mu \mathrm{m}$ & $20 \mu \mathrm{m}$ & $30 \mu \mathrm{m}$ & $40 \mu \mathrm{m}$ & $50 \mu \mathrm{m}$ & $60 \mu \mathrm{m}$ \\
\hline \multirow{2}{*}{1} & Simulation & 599.66 & 1198.6 & 1797.6 & 2396.6 & 2995.5 & 3594.3 \\
& Thin Plate & 599.76 & 1199.5 & 1799.3 & 2399.1 & 2998.8 & 3598.6 \\
\hline \multirow{2}{*}{2} & Simulation & 1247.7 & 2493.9 & 3740.2 & 4986.3 & 6232.1 & 7477.4 \\
& Thin Plate & 1246.5 & 2493.0 & 3739.6 & 4986.1 & 6232.6 & 7479.1 \\
\hline \multirow{2}{*}{4} & Simulation & 2047.4 & 4091.9 & 6136.4 & 8180.3 & 10223 & 12265 \\
& Thin Plate & 2046.6 & 4093.2 & 6139.8 & 8186.4 & 10233 & 12280 \\
\hline \multirow{2}{*}{6} & Simulation & 2335.0 & 4666.6 & 6998.0 & 9328.8 & 11658 & 13987 \\
& Thin Plate & 2336.8 & 4673.6 & 7010.4 & 9347.2 & 11684 & 14021 \\
\hline \hline
\end{tabular}




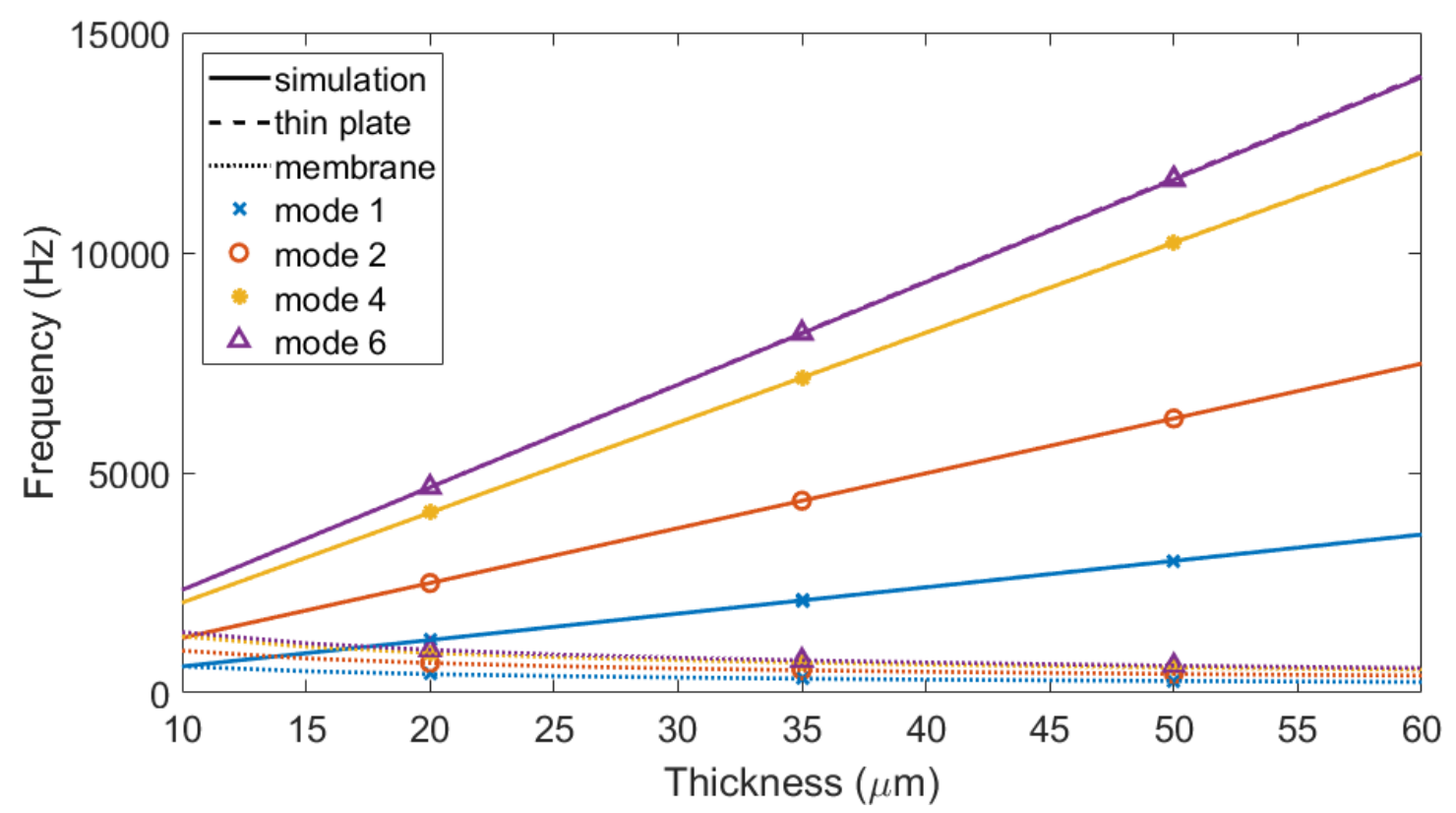

Figure 2.3: Numerical and analytical models (for both thin plate and membrane) of circular aluminum film (no battery coating) with a fixed boundary, radius of $0.65 \mathrm{~cm}$, and varying thickness. The first four unique resonance mode shapes are shown. The simulation results (solid line) correspond very closely with and therefore obscure corresponding thin plate analytical model results.

In contrast, the simulations demonstrate that the layered configuration of the battery electrode sometimes acts somewhat like a thin plate, but more often like a membrane. For the battery film simulations, with the two-layer structure, one of the battery coating parameters (thickness, density, or Young's modulus) was varied for each set of results while the others were held constant. These comparisons are given in Figs. 2.4-2.6. Unlike the aluminum model, the simulations of battery films show the resonance frequency decreasing with increasing coating thickness, demonstrating more membrane-like behavior. This is illustrated in Fig. 2.4, where the resonance frequency decreases as the coating thickness increases, and the simulations more closely correspond with the membrane model.

In Fig. 2.5 the coating thickness is at a constant value of $30 \mu \mathrm{m}$ and the coating density is varied. As expected from both simplified models in Eqs. (2.2) and (2.4), an increase in density corresponds to a decrease in resonance frequency for all modes. The numerical model results still match more closely with the analytical model for membranes. 


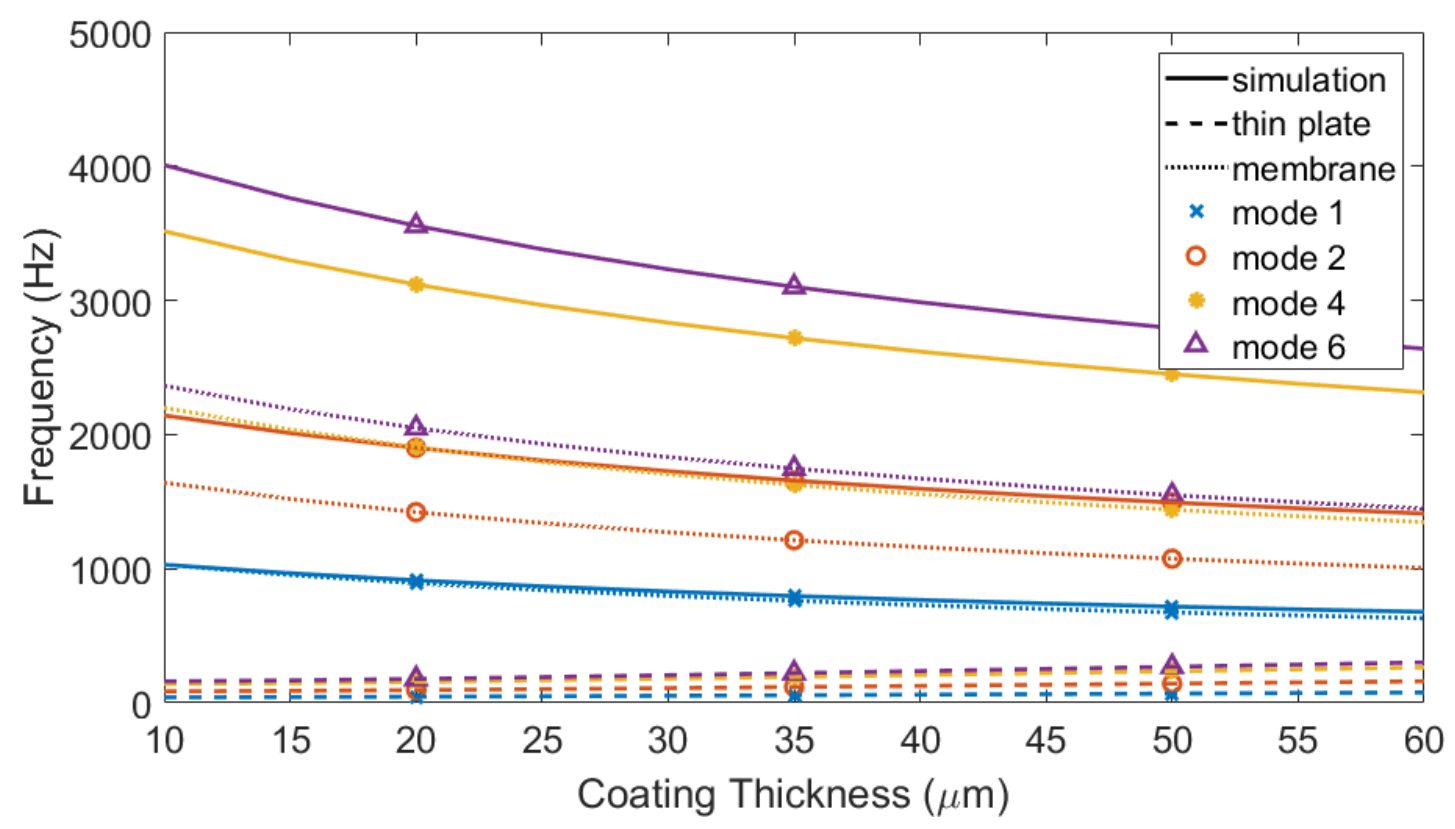

Figure 2.4: Numerical and analytical models of circular battery cathode with a fixed boundary, radius of $0.65 \mathrm{~cm}$, and the material properties listed in Table 2.4 . The thickness of the coating was varied to find the resonance frequencies for four mode shapes.

When simulating different Young's moduli, as shown in Fig. 2.6, an increase in the Young's modulus of the coating layer causes an increase in resonance frequency. This is expected from the forms of Eqs. (2.2) and (2.3). In this case, the simulated results do not match as closely with either thin plate or membrane theory, since the membrane theory does not rely on the Young's modulus.

Comparing the numerical and analytical models for a two-layer battery electrode film shows the limitations of using only analytical expressions to estimate the resonance frequencies. The simple models do not accurately capture the behavior of the vibrating structure when it has two layers. This disparity only worsens as the complexity of the system increases. Because of this, numerical simulations must be used to predict the vibration response. The numerical and simplified analytical models do not always correlate well, but experiments on real films can validate or refine the theoretical analysis. The combination of experimental results and numerical simulations can also lead to additional information by estimating the unknown parameters of the battery electrodes. 


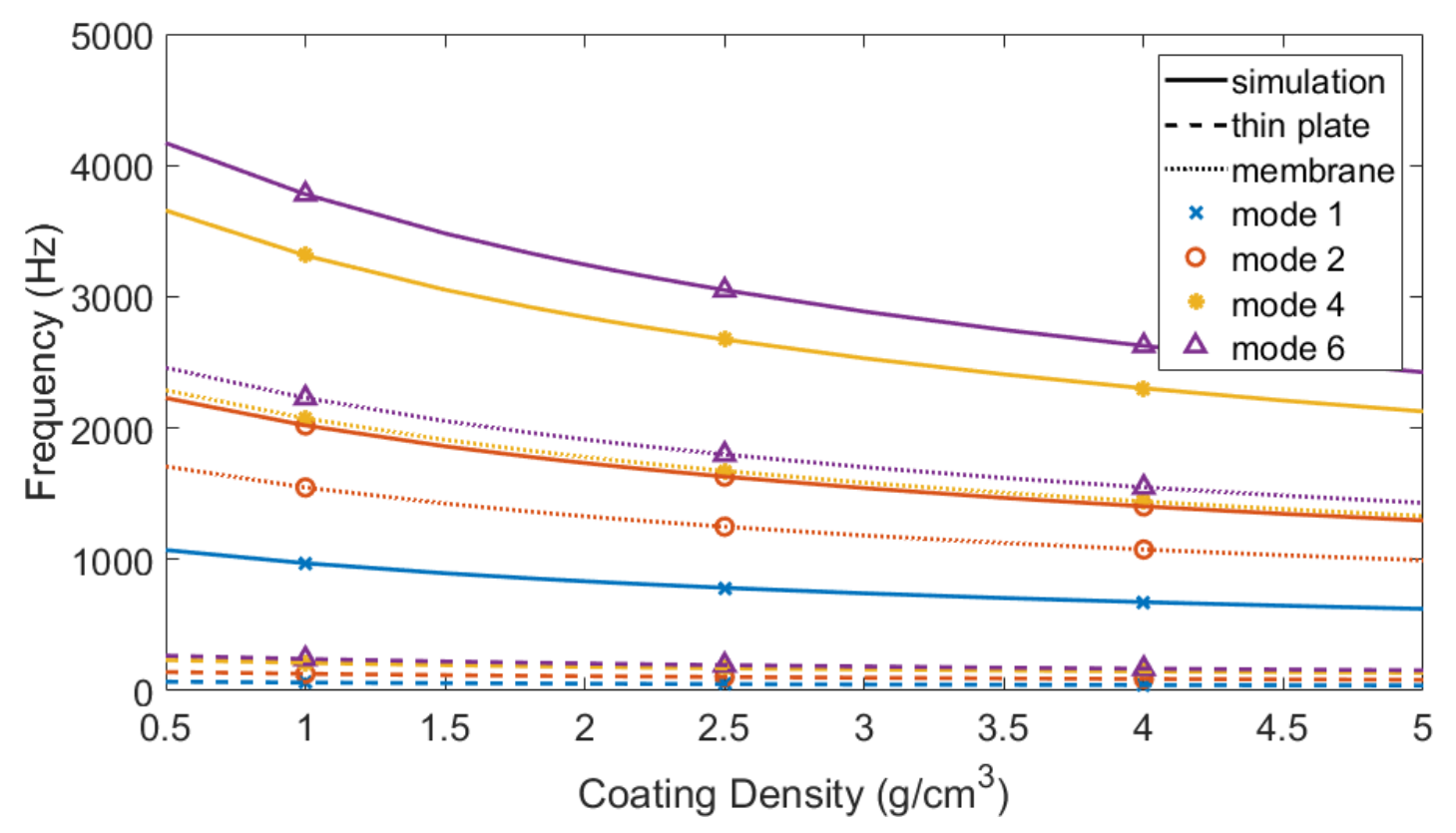

Figure 2.5: Numerical and analytical models of circular battery cathode with a radius of $0.65 \mathrm{~cm}$, and the material properties listed in Table 2.4. The density of the coating was varied to find the resonance frequencies for four mode shapes.

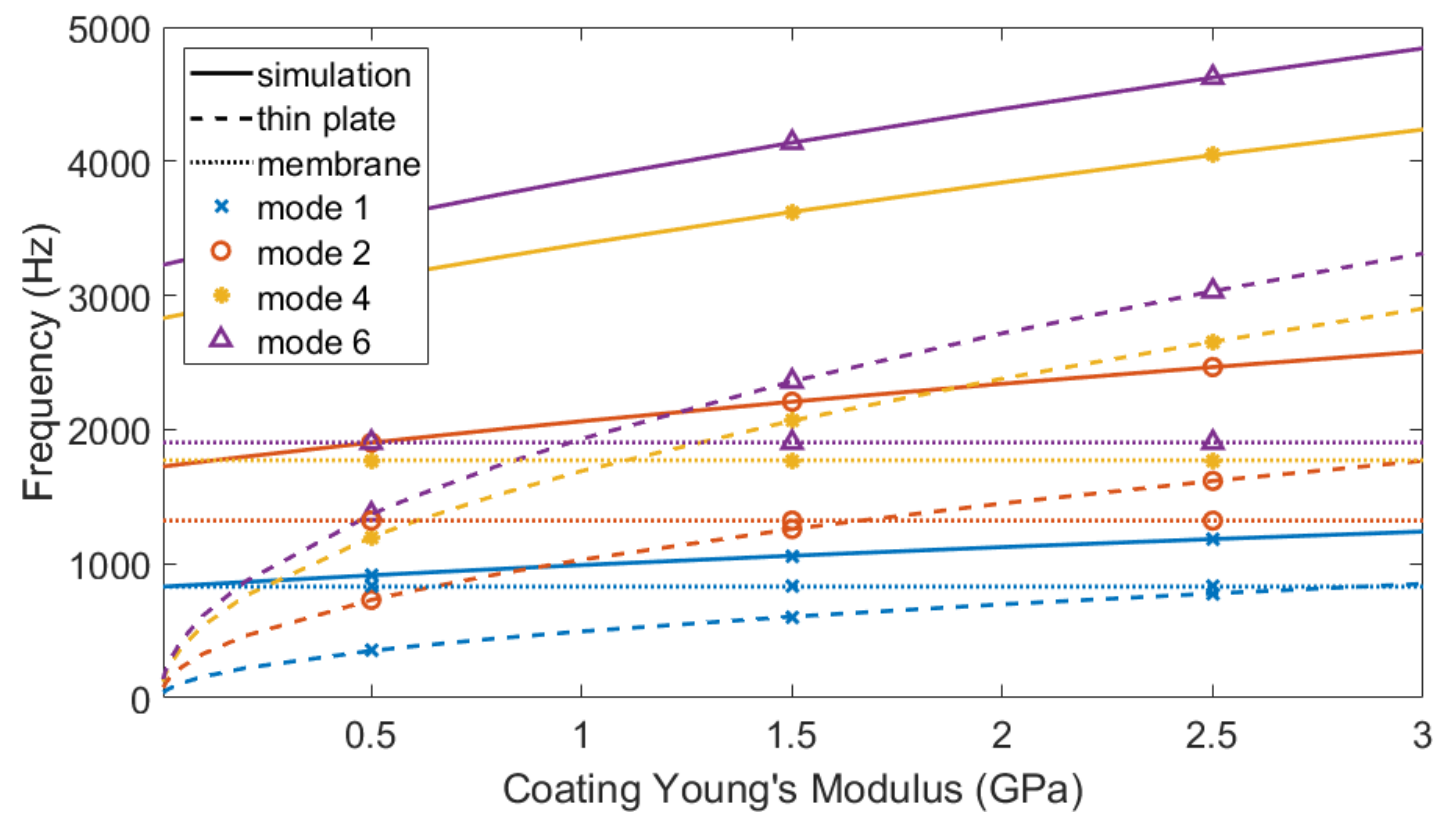

Figure 2.6: Numerical and analytical models of circular battery cathode with a radius of $0.65 \mathrm{~cm}$, and the material properties listed in Table 2.4. The Young's modulus of the coating was varied to find the resonance frequencies for four mode shapes. 


\section{CHAPTER 3. EXPERIMENTAL METHODS}

Although the theory explained in the previous chapter seems simple, implementation turned out to be more difficult. The first section in this chapter briefly describes some of the setups that did not work well or at all and how they led us to the method we eventually used. The later sections give details of the final apparatus used to obtain the results in this thesis.

\subsection{Preliminary Setups}

When taking resonance measurements, the apparatus consisted of a clamp to confine the vibrating area of the film, an excitation source, and a microphone for detection. Each of these components went through multiple iterations before we settled on the parts used in the final setup. Both iterations of the excitation source (acoustic and laser) were used to obtain the final measurements and will be addressed in the next section. The main problems we had to overcome were not being able to detect a response, damaging the films, and getting consistent and repeatable results.

A clamp was needed to securely confine the edges of the film to obtain a circular area with a clamped boundary. As seen in Eqs. (2.2)-(2.4), different boundary conditions, tension, and area will change the resonance response of the material, so we ensured that these were consistent between experiments.

We went through five main iterations of clamps before finding the final two we ended up using. The final clamps are described in detail in the next section. First we started with thin metal plates bolted together, but the sheets were thin enough that drilling the hole through them warped the plates, so they could not effectively clamp the film. Our second try with thicker metal plates worked better, but the metal wasn't smooth enough to completely clamp the film, and it was difficult to get the edge around the drilled hole completely even. Both of these clamps are shown in Fig. 3.1. We also tried making several versions of a vacuum clamp out of acrylic and o-rings, as seen in Fig. 3.2. This did not work, mainly because of the poor quality of the vacuum. The vacuum 


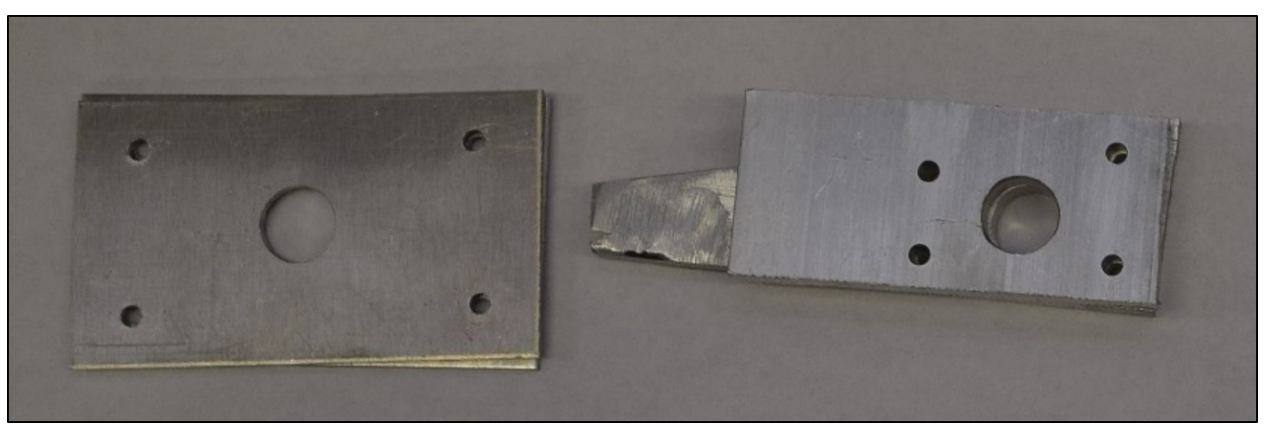

Figure 3.1: Photo of the first two clamps tried. Both were made of metal plates held together by nuts and bolts.

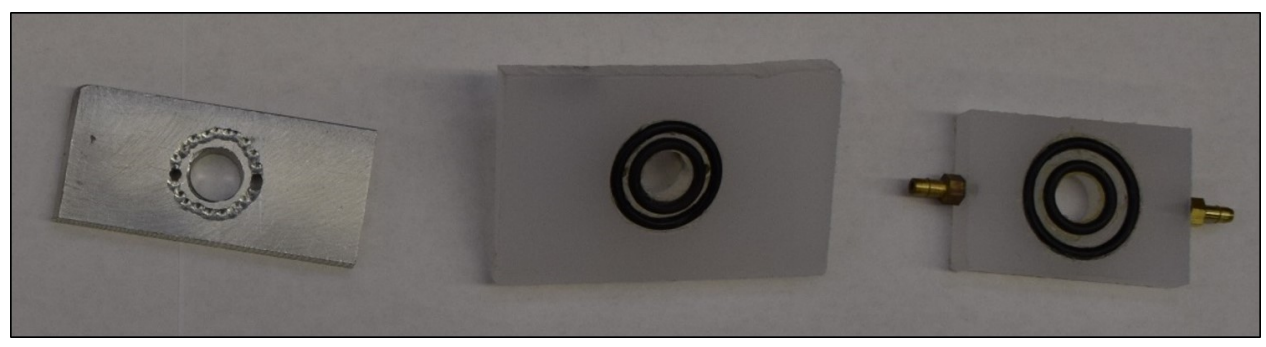

Figure 3.2: Photo of several versions of the clamps used with the vacuum. Although confining the films with the vacuum was unsuccessful, these clamps led us to one of the final clamps.

wasn't constant or strong enough and added too many other vibrations. However, this setup led us to one of our final clamps, made of acrylic. We tried using pipe flanges as the fourth type of clamp. The idea was that the flanges would be the same size and easy to align (by putting pins through the outer holes). There ended up being two main problems with the flanges: they weren't as smooth as we had anticipated, and we couldn't find any that were small enough. Our detection isn't as reliable in the very low $(<500 \mathrm{~Hz})$ range, and a smaller area gives a higher resonance frequency, so we wanted a diameter no larger than half an inch. Unfortunately, the smallest flanges of the right type that we found were for half inch pipes, meaning the inner diameter of the hole was actually $2.239 \mathrm{~cm}$ (0.8815 inches). The fifth clamp was a laser-cut acrylic clamp tightened with nuts and bolts. Along with being easily warped, this clamp had the same surface roughness problem as the earlier metal clamps. These last two clamps are shown in Fig. 3.3.

We took a detour in the middle of our resonance experiments to try surface acoustic wave (SAW) detection in an aluminum plate $(0.65 \mathrm{~cm}$ thick) and in aluminum foil (several types ranging in thickness from $9 \mu \mathrm{m}$ to $26 \mu \mathrm{m}$ ). We tried several combinations of excitation and detection 


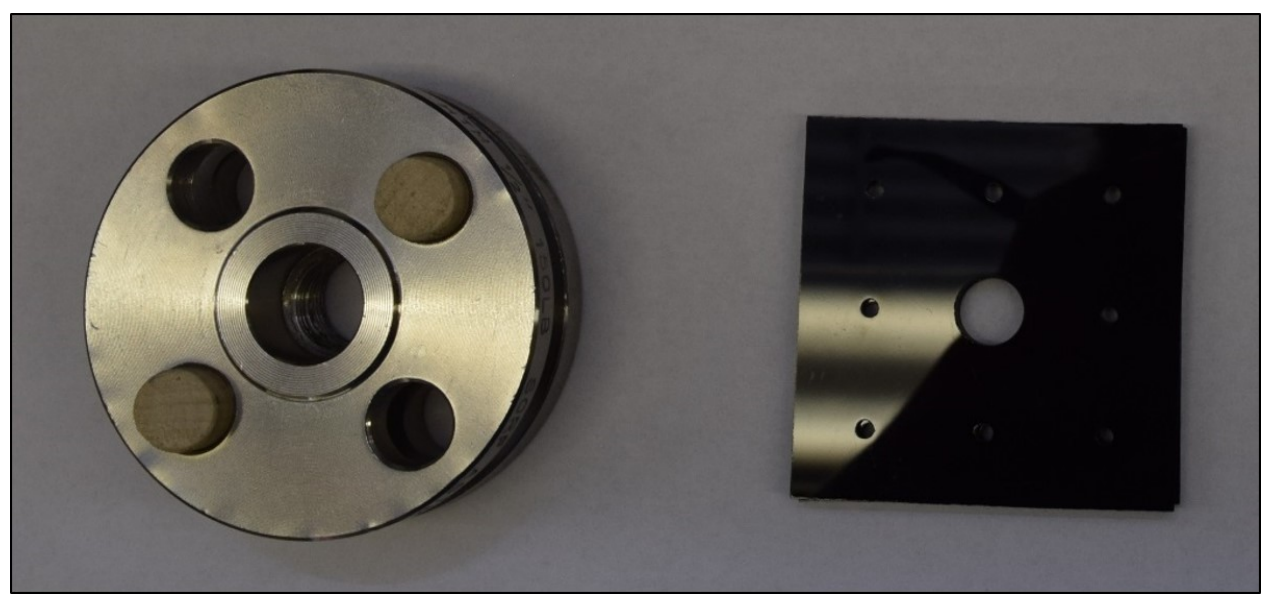

Figure 3.3: Photo of the fourth and fifth versions of the clamps. On the left is the clamp made up of half inch flanges, which ended up being too large. The clamp on the right is the laser-cut acrylic, which wasn't smooth enough and warped too easily.

sources: sparkgap and piezoelectric element (piezo), sparkgap and microphone, piezo and piezo, speaker and piezo, speaker and microphone, laser and piezo, and laser and microphone. The setup with aluminum foil and piezos for both excitation and detection is shown in Fig. 3.4.

The sparkgap, which generates a spark between two wires and produces a loud noise, excited the aluminum foil well, but it was difficult to control when it triggered, so we did not continue with that as the excitation source. With the microphone, all we picked up was the signal coming from the source through the air, so we quickly abandoned that method of detection. The piezos were able to pick up vibrations moving through the aluminum with either excitation source, but the piezos worked better for excitation as well, since they were physically coupled to the plate or foil. However, piezos have a narrow frequency response range, making them less versatile than the speaker.

Unfortunately, we do not believe that we were ever able to detect an actual SAW in the aluminum. Rather, we were just detecting the flexural vibrations of the aluminum that settled into a resonance. There were two good outcomes, however. One was that this got us thinking about exciting resonances by using mode coupling, where the material is excited at an off-resonance frequency but then settles into its natural frequency. The other was that we started using a pulsed infrared laser for better excitation. We still didn't detect the SAWs with the piezos, but using the laser ended up working for our resonance tests as well. While we never successfully implemented 


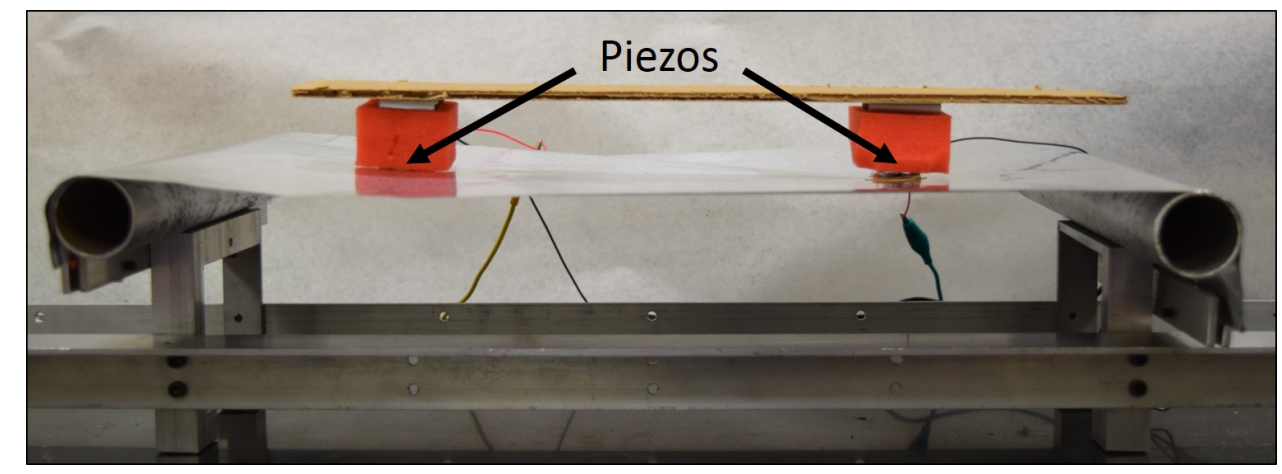

Figure 3.4: Photo of the SAW detection setup on aluminum foil, with piezos for both excitation and detection. The foil is spread over two rollers and clamped on each side. The tension can be adjusted by changing the amount of weight on the clamped edge.

SAW detection as a method for characterizing a material, it is possible. Using SAWs for material interrogation is a well-documented method, as mentioned in Chapter 1. With better equipment for detection, we should be able to do this as well.

We tried using several different microphones for detection, which can be seen in Fig. 3.5. Initially, all of our tests were done using a free-field, 1/4 inch microphone (130E20 from PCB Piezotronics) in combination with a National Instruments signal acquisition module (NI 9234). However, when we switched to using the laser, this microphone was not as useful because we could not easily trigger the microphone with the laser pulse. It was also limited in how fast it could sample, with a maximum sampling rate of $51.2 \mathrm{ksamples} / \mathrm{s}$. We then tried using an ultrasonic microphone (Ultramic 250K from Dodotronic), which can detect frequencies up to $125 \mathrm{kHz}$. Even though this microphone could sample faster and detect higher frequencies, ultimately we decided not to use it because of the same lack of triggering we had with the NI microphone setup. We decided to use a back electret microphone (WM-61A from Panasonic Electronic Components) because it could be easily controlled via the Digilent Analog Discovery, which we also used to control the laser. This way both laser and microphone could be triggered simultaneously. The setup of the Analog Discovery software also made it easy to repeat measurements quickly, allowing us to average many tests together. Being able to average tests helped us overcome the problem of lower sensitivity with this microphone, since we could attain a better signal-to-noise ratio using averaging. In addition, the Analog Discovery can sample at rates up to $100 \mathrm{Msamples/s,} \mathrm{making} \mathrm{it}$ significantly faster than the other components. 


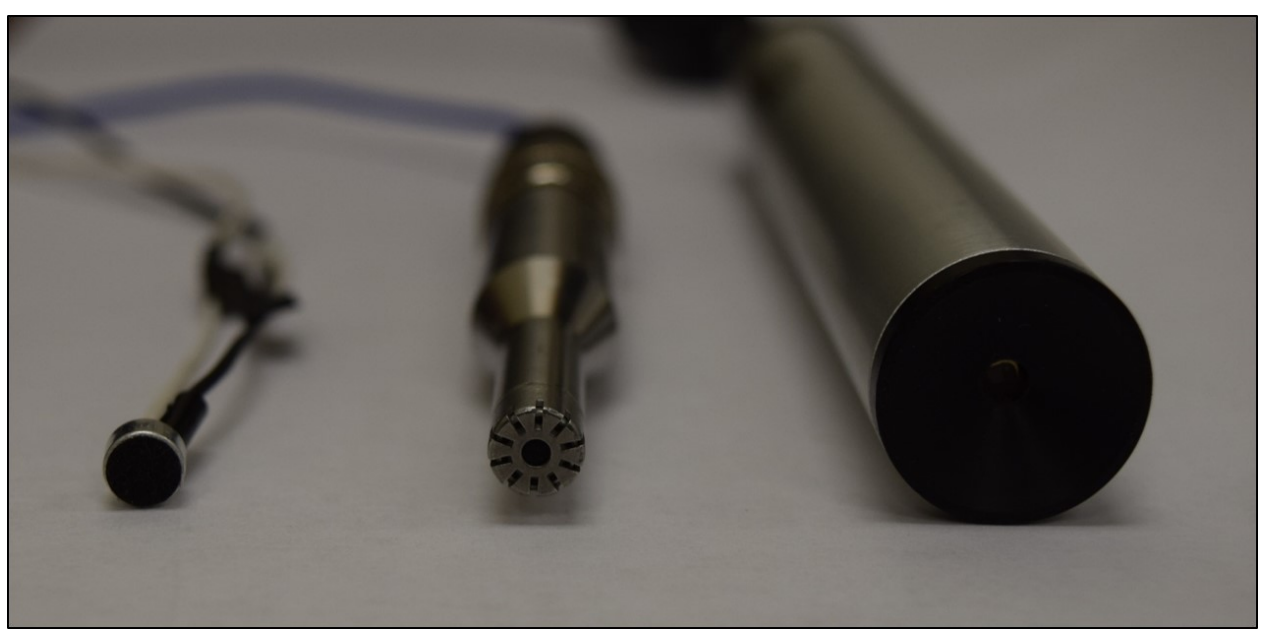

Figure 3.5: Photo of the three microphones used. From left to right, the electret microphone used in the final setup, the free-field microphone used with the NI module, and the ultrasonic microphone.

The setup used to take the resonance measurements changed many times in an attempt to get better and more repeatable results. However, the process of trying different components led us to an apparatus that worked well. This setup, which was used to take the results given in this thesis, is described in the next section.

\subsection{Experimental Apparatus}

The measurement apparatus consisted of a custom clamp to confine the vibrating area of the film, an excitation source, and a microphone on the opposite side of the clamp. The excitation source was either a pulsed laser, as shown in Fig. 3.6, or a speaker, as shown in Fig. 3.7. When orienting the films so that the coating side was facing the laser, microscope slides (each $1.2 \mathrm{~mm}$ thick) were also placed between the laser and the clamped film in order to attenuate the laser sufficiently to avoid ablation of the film coating.

We used two different clamps for the final experiments. The first clamp was made of two pieces of acrylic (each $0.46 \mathrm{~cm}$ thick) with an o-ring (AS568 size 112, with $0.264 \mathrm{~cm}$ cross-section, $1.265 \mathrm{~cm}$ inner diameter, and $1.775 \mathrm{~cm}$ outer diameter) around the hole (diameter $1.296 \mathrm{~cm}$ ) drilled through each piece. The clamp aligns the two o-rings to ensure the film is firmly held in place, with the acrylic sides held in place by and tightened with nuts and bolts. The film was placed between the two o-rings. Because this clamp used bolts to apply the clamping, rather than weight, it could 


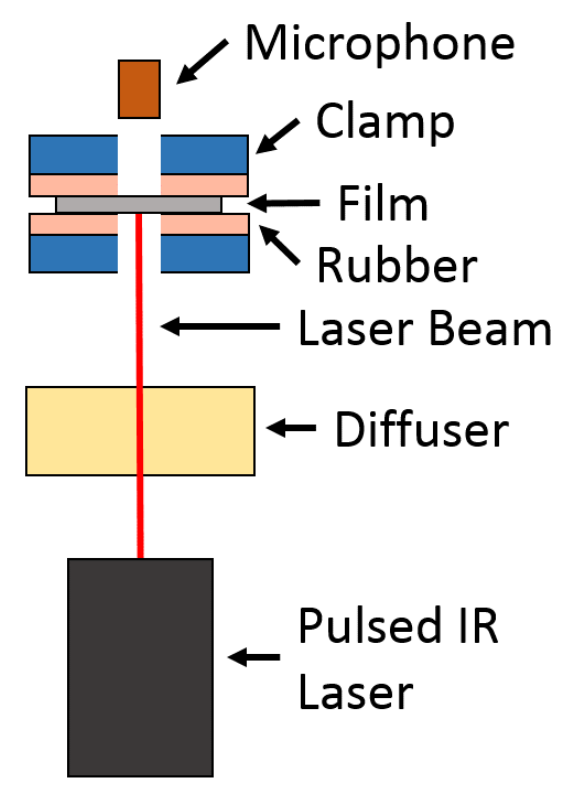

Figure 3.6: Illustration of the cross-sectional view of the setup for the resonance measurements using a laser for excitation. The laser beam excites a vibration in the area of the film confined by the clamp. A microphone behind the clamp records the acoustic response of the film. When the film is oriented such that the coating side is being hit by the laser, the beam is attenuated to avoid film ablation.

be difficult to get the same clamping every time because the bolts could be tightened different amounts, even to the point that caused warping of the acrylic pieces. To solve this, spacers were used between the acrylic pieces to keep them $0.5 \mathrm{~cm}$ apart so that the bolts could only be tightened to a certain point, helping to keep the clamping consistent between tests. The hole drilled through the acrylic was $1.3 \mathrm{~cm}$ in diameter, but the o-rings, which actually confine the film area, had a slightly larger diameter of $1.411 \mathrm{~cm}$ from compressed edge to edge, so this was the dimension of the actual vibration area. This clamp is shown in Fig. 3.8.

The second clamp was made of two steel plates $(7.6 \mathrm{~cm} \times 7.65 \mathrm{~cm} \times 0.4 \mathrm{~cm})$ with a hole (diameter $1.28 \mathrm{~cm}$ ) drilled through the middle of each for the vibration area. Steel pins (length $2.85 \mathrm{~cm}$, diameter $0.4 \mathrm{~cm}$ ) were fixed into the corners of the bottom plate, and corresponding holes (diameter $0.5 \mathrm{~cm}$ ) were drilled in each of the four corners of the top plate for alignment. Rubber sheets (1.25 mm thick, durometer Shore 19A) were placed in between the steel plates to make sure full contact was made with the film and weights $(885.6 \mathrm{~g}$ or $1253.6 \mathrm{~g}$ ) were placed on top to 


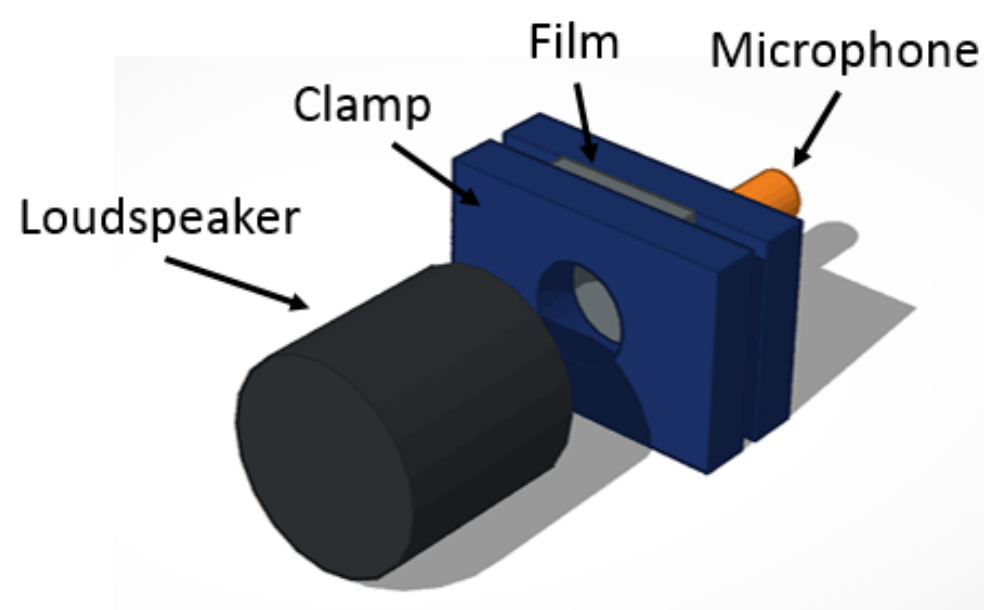

Figure 3.7: Illustration of the setup for the resonance measurements using a speaker for excitation. The speaker excites a vibration in the area of the film confined by the clamp. A microphone behind the clamp records the acoustic response of the film. The setup could be oriented as shown (for the acrylic clamp only), or rotated with the clamp and microphone above the speaker (for either clamp).
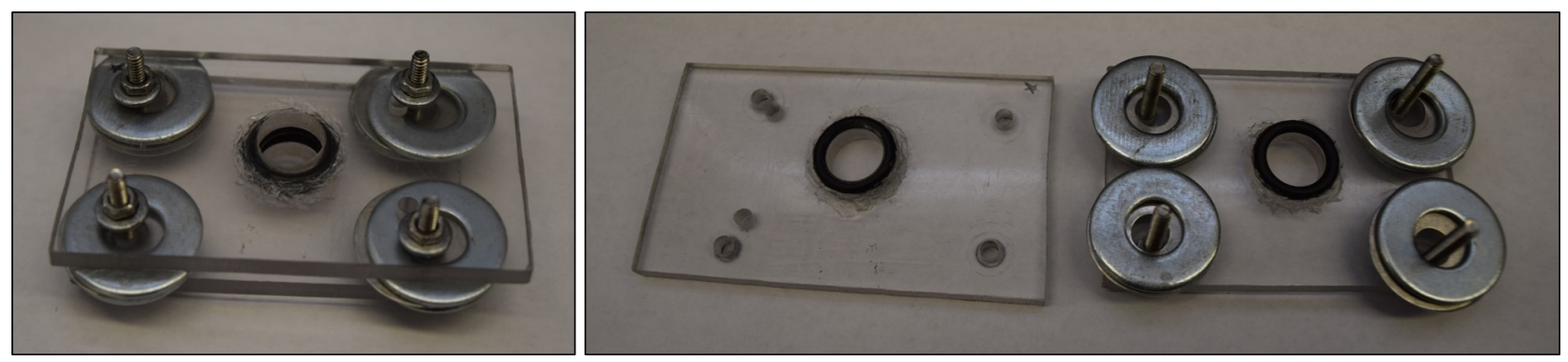

Figure 3.8: Photos of the acrylic clamp with o-rings used to confine the vibration area of the films.

provide full clamping of the circumference of the vibration area. This clamp is shown in Fig. 3.9 and as part of the full setup in Fig. 3.10.

Different excitation sources have advantages and disadvantages, leading us to analyze results from two different sources. The acoustic source, a loudspeaker (808 Canz speaker for the resonance tests), cannot couple as much energy into the film as the high-power laser, but it is more versatile and allows for better control of the excitation signal. The user can easily control what signal is input to the speaker, allowing for a variety of signal shapes and frequencies, such as a sine wave or a frequency sweep. However, when using an acoustic source, the signal detected by the microphone will include the sound generated by the source in addition to the desired resonance. 

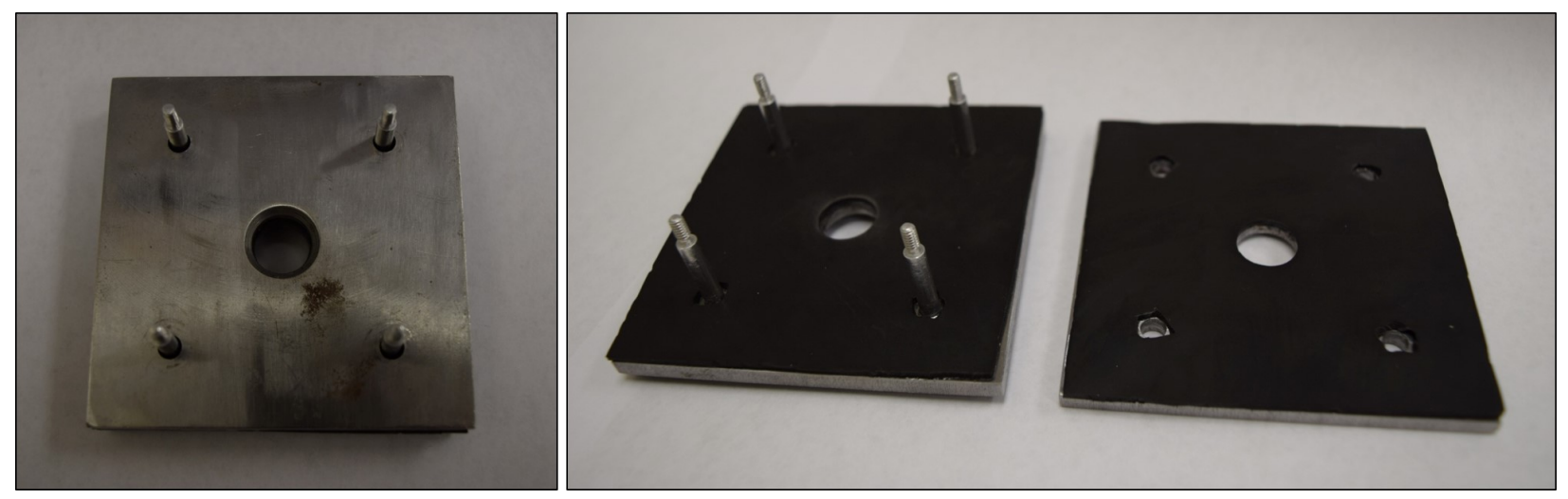

Figure 3.9: Two views of the steel clamp used to confine the vibration area of the films.
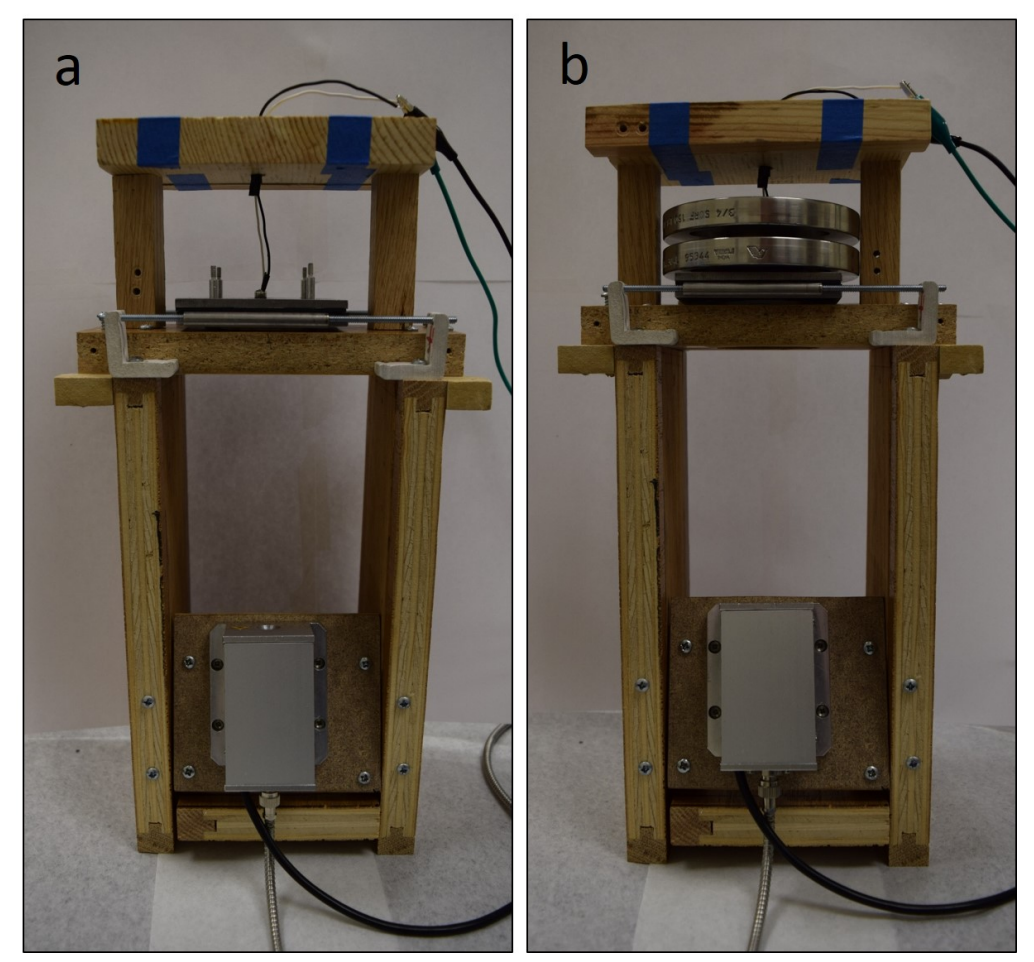

Figure 3.10: Photo of the full setup with the steel clamp shown in Fig. 3.9, without (a) and with (b) weights.

This can be problematic if the two overlap. With the laser, there is no sound from the source, so the only sound in the measurement is that generated by the movement of the film. This eliminates any uncertainty as to the source of sound in our microphone measurements while still exciting sufficient vibrations in the battery film. The laser also transfers more energy into the film. One disadvantage, though, is that the excitation frequency cannot be changed without using something like 

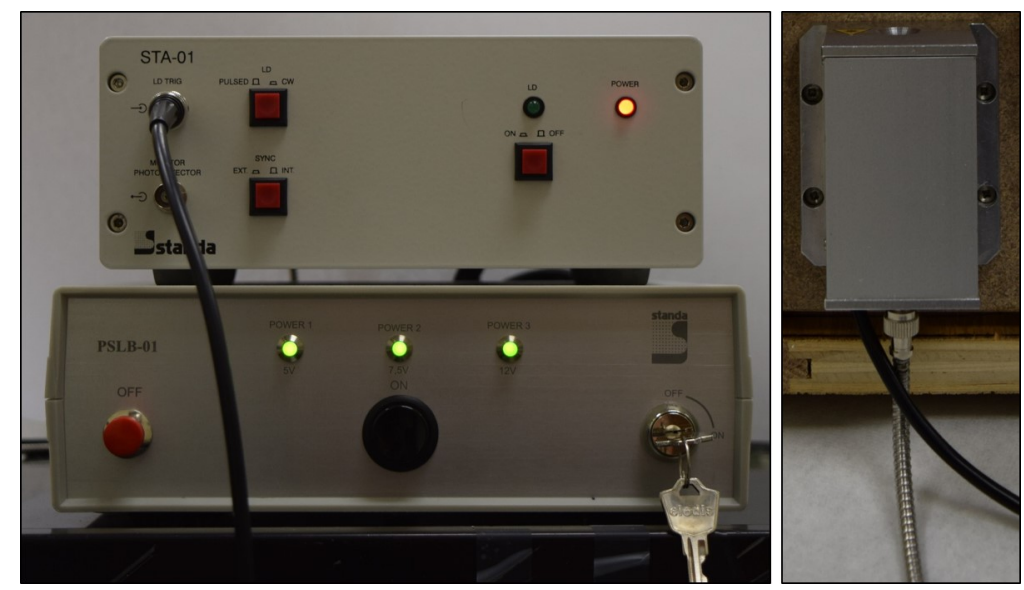

Figure 3.11: Photo of the pulsed infrared laser used in the setup shown in Fig. 3.6.

a diffraction grating to create multiple interfering excitation sources. A laser with this much power also requires certain safety protocols, which makes it less convenient than an acoustic source.

\subsection{Laser}

When using a laser as the excitation source, the pulsed laser beam excites the film by briefly heating it up [29], [30]. This in turn creates a vibration in the material. We used a pulsed infrared laser from Standa (model STA-01-8-1053). This laser has a wavelength of $1053 \mathrm{~nm}$ and can be turned on via an external trigger for short bursts (about 500 ps in length). The reflectivity of the material at the wavelength of the laser can also be problematic. Aluminum reflects infrared light very well (smooth aluminum has over $90 \%$ reflectivity, and rough aluminum can have over 50\% reflectivity [52]), so it does not absorb much of the laser energy. When testing our setup with aluminum foil, which is very similar to the current collector layer on our films, insufficient energy was being absorbed to excite a large vibration. When we switched to battery films (with a coating which, unlike aluminum, is dark and not shiny) we found that too much energy was being absorbed and the film was being damaged. Thus different materials require different levels of attenuation for the laser in order to avoid damage, and materials that are too reflective at the laser wavelength may need some sort of coating that absorbs the energy. Because of this, iteration is necessary to determine the amount of energy needed to transfer enough energy to the film without damaging it. 


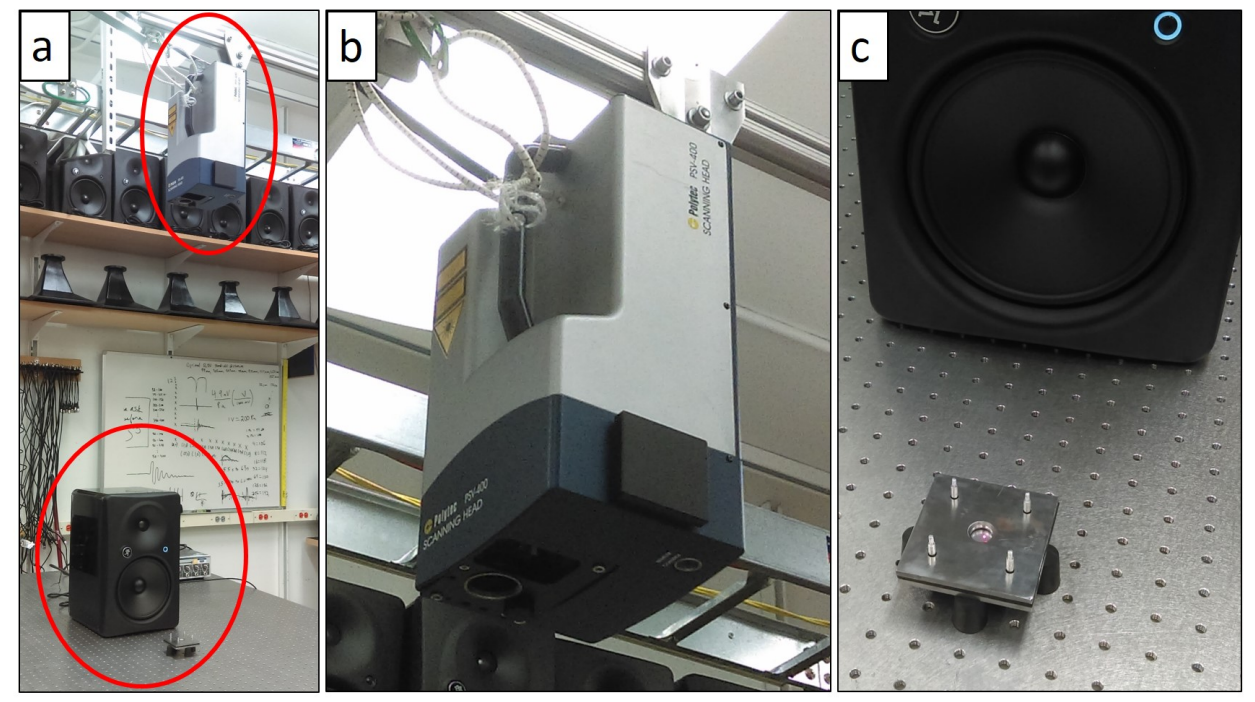

Figure 3.12: Photo of the setup for the scanning laser Doppler vibrometer measurements. a) The full setup, with the SLDV, clamp, and speaker circled. b) The SLDV. c) The speaker and clamp, with the laser shining on the clamped film.

\subsection{Scanning Laser Doppler Vibrometer}

To ensure that the peaks we were obtaining from our measurements were indeed the fundamental frequencies of each film, we used a scanning laser Doppler vibrometer (SLDV) to examine the clamped cathodes. For the SLDV measurements, the films were excited with a large speaker playing a frequency chirp from $100 \mathrm{~Hz}$ to $5 \mathrm{kHz}$ and the resulting velocity of the films was measured using a Polytec PSV-400 scanning vibrometer. The SLDV works by aiming a laser at the object being examined and measuring the Doppler shift of the reflected light in order to obtain the velocity of the object. By moving the laser around to different points on the object, we can get a map of how the object is vibrating [53]. For each film, we had 60-100 locations for the laser and took an average of 5 chirps to obtain the data for each location.

\subsection{Processing}

The acoustic response was recorded at a sampling frequency of $100 \mathrm{kHz}$ using a back electret condenser microphone located on the opposite side of the film from the excitation. The laser was externally triggered using a Digilent Analog Discovery, which also collected the microphone data via an oscilloscope channel and provided the voltage for the microphone. For each of the 
data sets shown here, many tests were averaged together to increase the signal-to-noise ratio. The battery film spectra are the result of 1000 tests for each film, while the drying results are each an average of 200 tests. The Fast Fourier Transform (FFT) of this averaged data was computed to find the frequency response, and from this, the power spectral density was calculated.

For the tests that used the PCB microphone with the NI module, the data was not averaged. Because these tests had acoustic excitation using a frequency sweep, the relative power spectral response was calculated by summing the squares of the raw microphone data over each frequency in the sweep, similar to an FFT.

Using the apparatus and processing methods described in this chapter, we were able to perform experiments on battery electrodes and aluminum films and compare the results to the simulated and theoretical predictions. The next chapter summarizes these results. 


\section{CHAPTER 4. RESULTS}

With the apparatus described in the previous chapter, results were obtained for three commercialgrade battery cathodes with different coatings, as well as aluminum films with dried and drying layers of paint.

\subsection{Battery Films}

The averaged microphone measurements (from 1000 tests) of three different cathodes clamped in the steel clamp (Fig. 3.9) and a noise reading are shown in Fig. 4.2. The acoustic spectra computed from these measurements are shown in Fig. 4.3. The resonances of three cathodes with a $20 \mu \mathrm{m}$ aluminum current collector are seen to occur at different frequencies. Each of the cathodes had coating layers of different thicknesses $(26 \mu \mathrm{m}, 38 \mu \mathrm{m}$, and $42 \mu \mathrm{m}$ coatings on top of the current collector) and densities. A baseline reading (Fig. 4.2d) was taken with the laser off to determine what frequencies, if any, were caused by external noise in the room. This baseline is also included in Fig. 4.3 to demonstrate that the peaks shown are not due to room noise. The resonances of the same three cathodes in the acrylic clamp (Fig. 3.8) are seen in Fig. 4.4. For both sets of resonance tests, the pulsed laser was the excitation source.

\subsection{Scanning Laser Doppler Vibrometer}

In order to see what modes were being excited in the films, some measurements were taken with an SLDV. When the SLDV has completed a full scan, it finds the response spectrum of the system. The user can then select portions of the spectrum to see how the system was moving in that frequency range, and the SLDV outputs a map of the velocity of the system in that range. The maps shown here are a single time frame taken near the peak amplitude of the film oscillation. Note that they have different amplitude scales, but the displacement is very small compared to the diameter

of the clamp and has been exaggerated here to show differences between modes. The frequency 
Table 4.1: Properties of the three battery films tested. The composition lists the main component in the coating. The total $h$ is the combined thickness of the aluminum current collector and the coating layer, as reported by the manufacturer and as measured by us. The density listed was calculated from the measured total $h$ (assuming all of the current collectors were $20 \mu \mathrm{m}$ thick), weight, and surface area of the film samples. Some of the samples do not have a density listed because they included the edge of the film, where the coating thickness can vary drastically.

\begin{tabular}{llccc}
\hline \hline Material & composition & $\begin{array}{c}\text { total } h(\mu \mathrm{m}), \\
\text { reported }\end{array}$ & $\begin{array}{c}\text { total } h(\mu \mathrm{m}), \\
\text { measured }\end{array}$ & $\begin{array}{c}\text { density }\left(\mathrm{kg} / \mathrm{m}^{3}\right), \\
\text { measured }\end{array}$ \\
\hline \multirow{2}{*}{ Coating 1 } & Toda NCM 523 & 62 & 68 & - \\
& & & 69 & 2360 \\
& & & 69 & - \\
Coating 2 & Toda 523 & \multirow{2}{*}{58} & 68 & 2396 \\
& & & 69 & - \\
& & & 65 & 2652 \\
& & & 65 & 2650 \\
Coating 3 & Toda HE5050 & 46 & 48 & 2682 \\
& & 46 & 52 & 2456 \\
& & & 49 & 2407 \\
& & & 49 & 2366 \\
\hline \hline
\end{tabular}
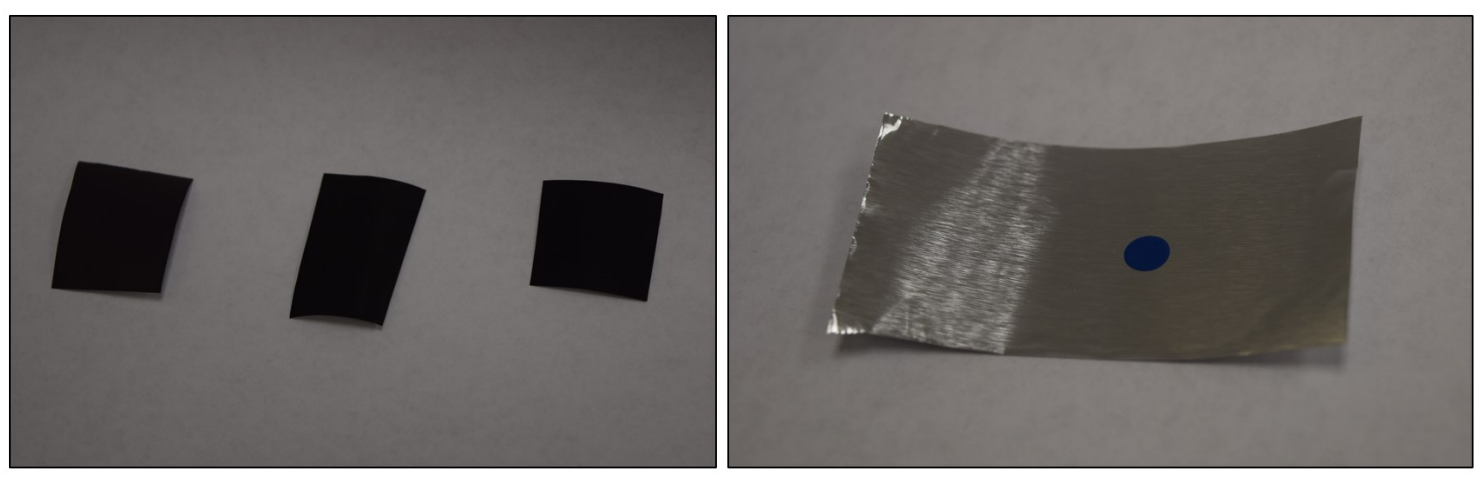

Figure 4.1: Photo of the three battery cathodes tested (left to right: $26 \mu \mathrm{m}$ coating, $38 \mu \mathrm{m}$ coating, $42 \mu \mathrm{m}$ coating) and aluminum foil with a drop of paint.

spectrum for the battery film with coating 1 clamped in the steel clamp is shown in Fig. 4.5. In the frequency range scanned $(100 \mathrm{~Hz}-5 \mathrm{kHz})$, there were three main peaks. Each of these was selected to create a velocity map in those frequency ranges. The map for the first peak (Fig. 4.6), occurring around $784 \mathrm{~Hz}$, looks like a fundamental mode (mode 1 from Fig. 2.1). The second 

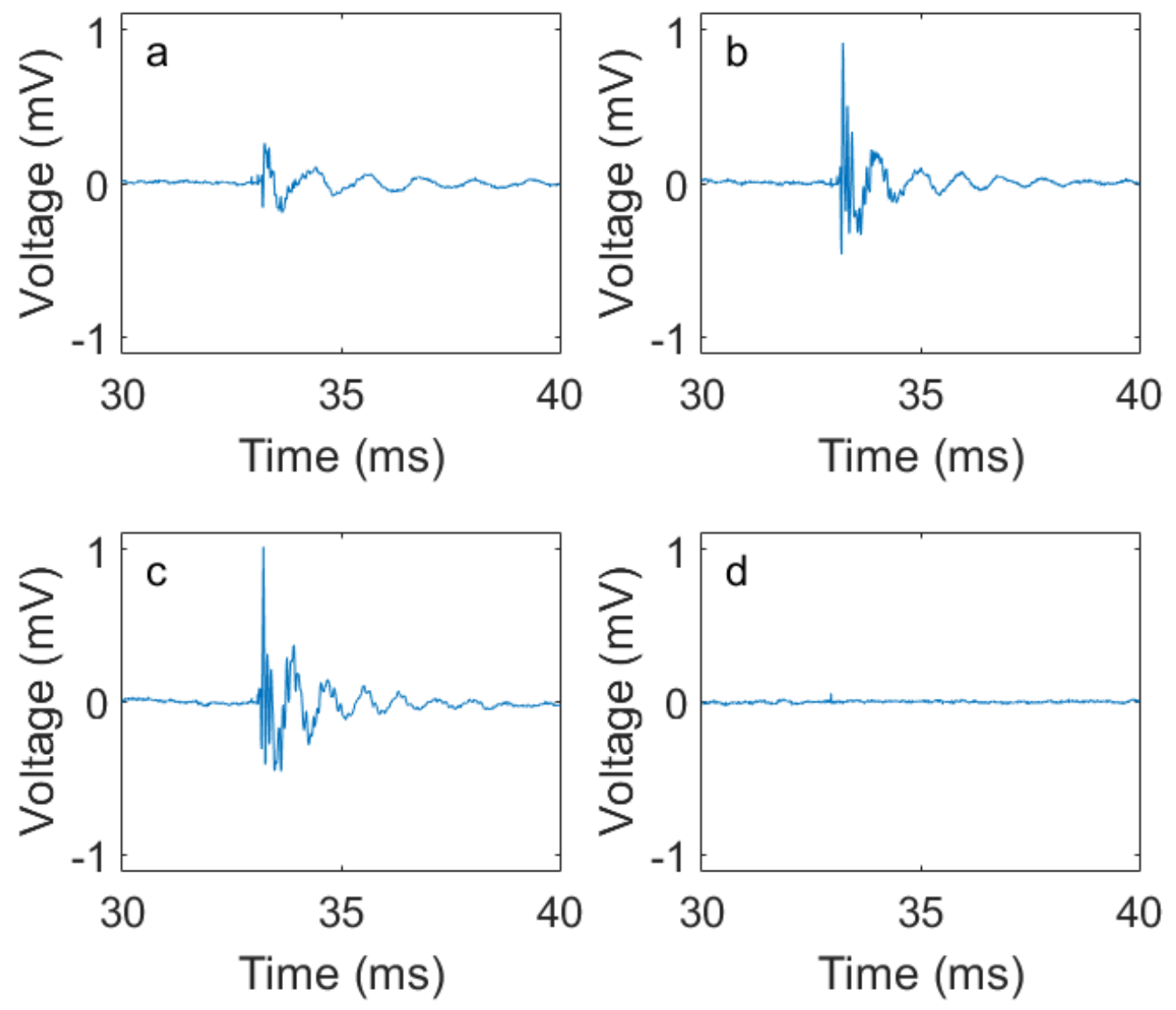

Figure 4.2: Time-domain measurements for various battery films clamped in the steel clamp as shown in Figs. 3.6 and 3.9. All of the films are cathode electrodes with different coating thicknesses on $20 \mu \mathrm{m}$ aluminum, and all measurements are an average of 1000 tests. a) $42-\mu \mathrm{m}$-thick coating, b) 38- $\mu \mathrm{m}$-thick coating, c) $26-\mu \mathrm{m}$-thick coating, d) baseline reading with laser off to get room/electrical noise reading for comparison.

peak, at $1806 \mathrm{~Hz}$, looks similar, as seen in Fig. 4.7. This peak was present for all of the films tested in the steel clamp. The map for the third peak, shown in Fig. 4.8, looks like the second symmetric mode (mode 6 from Fig. 2.1). This mode was not observed for the other two battery films. The spectrum and map for the first peak of the electrode with coating 2 are shown in Figs. 4.9 and 4.10. This peak occurred around $1019 \mathrm{~Hz}$, and the map at that frequency appears to be a fundamental mode, but the shape is slightly off-center. For the electrode with coating 3, the first peak is around $1281 \mathrm{~Hz}$ (Fig. 4.11), and it also looks like a fundamental mode (Fig. 4.12). An SLDV scan of a piece of notebook paper with reflective tape (to make it reflective enough for the SLDV to get a 


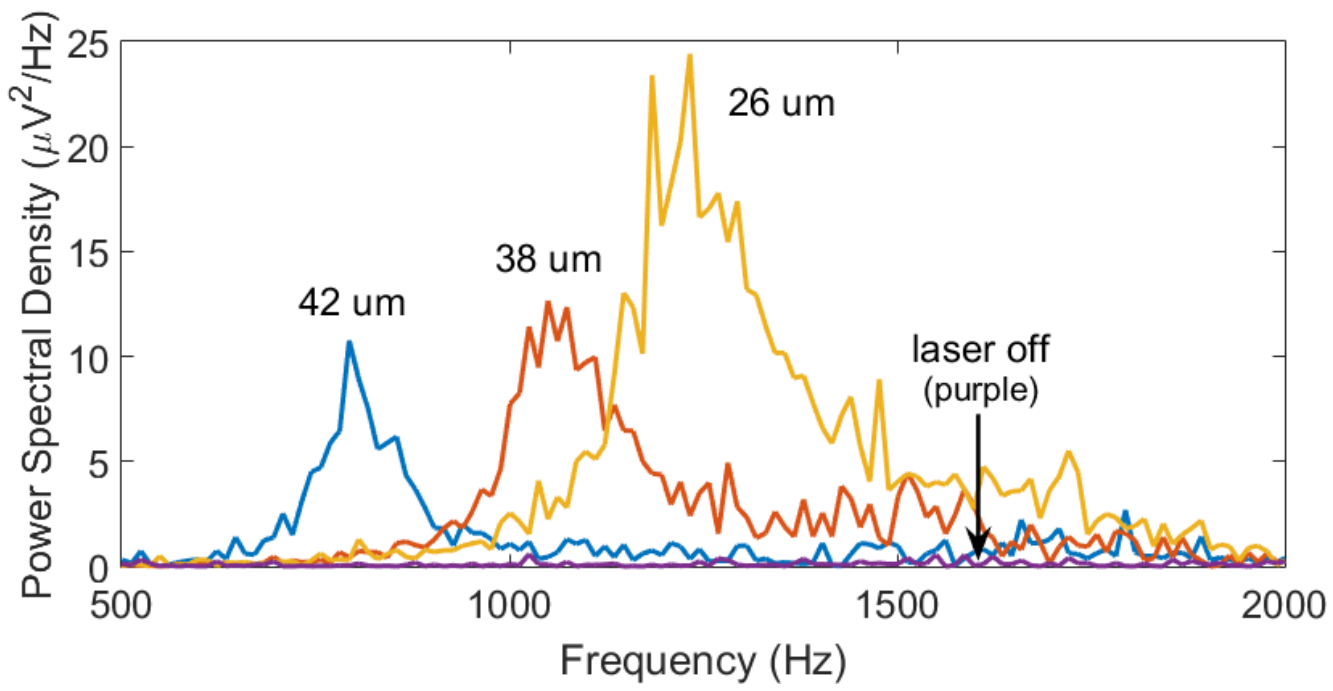

Figure 4.3: Power spectral density for various battery films clamped in the steel clamp as shown in Figs. 3.6 and 3.9. All of the films are cathode electrodes with different coating thicknesses (26 $\mu \mathrm{m}, 38 \mu \mathrm{m}$, and $42 \mu \mathrm{m}$ ) on $20 \mu \mathrm{m}$ aluminum. The purple line labeled "laser off" is a baseline reading to ensure that the peaks seen are not due to room noise. The spectra here were obtained from the time-domain measurements shown in Fig. 4.2.

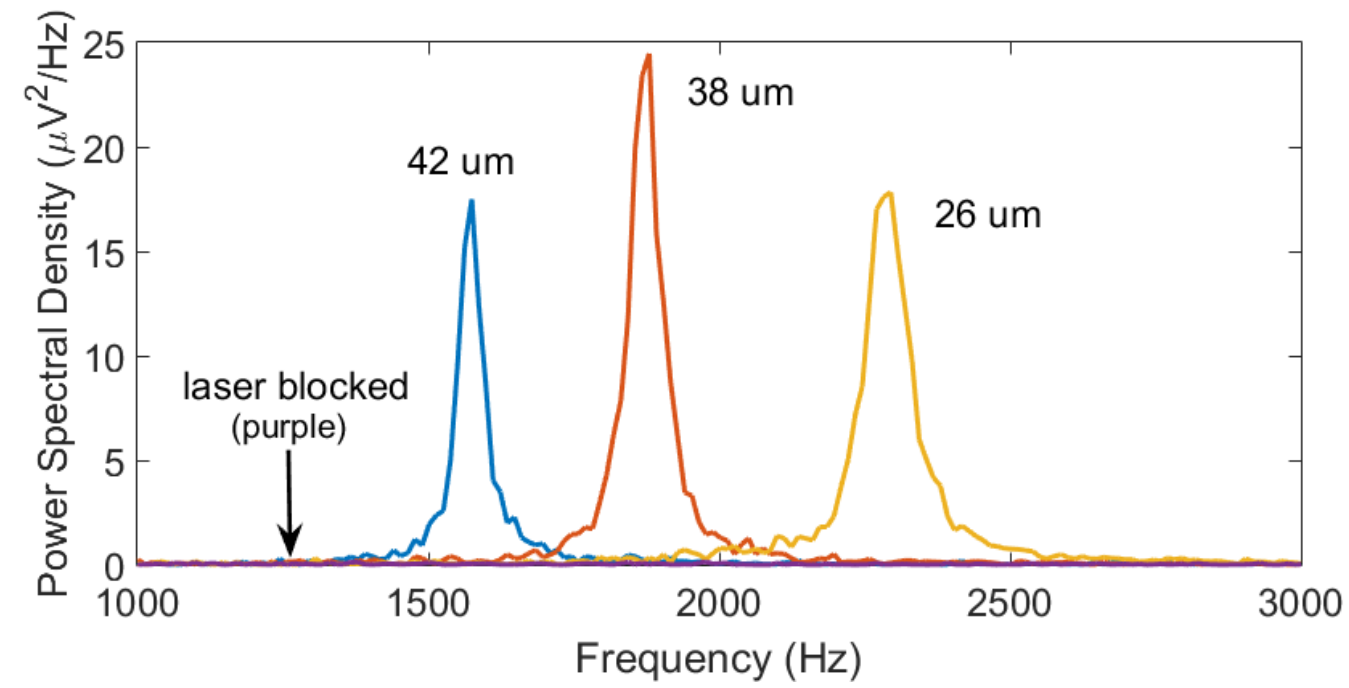

Figure 4.4: Power spectral density for various battery films clamped in the acrylic clamp as shown in Figs. 3.6 and 3.8. All of the films are cathode electrodes with different coating thicknesses (26 $\mu \mathrm{m}, 38 \mu \mathrm{m}$, and $42 \mu \mathrm{m}$ ) on $20 \mu \mathrm{m}$ aluminum. The purple line labeled "laser blocked" is a baseline reading to ensure that the peaks seen are not due to room noise.

reading) in the steel clamp was also taken, and the same $1800 \mathrm{~Hz}$ peak seen for the battery films was present there as well (see Figs. 4.13 and 4.14). A different film was tested in the acrylic clamp, 


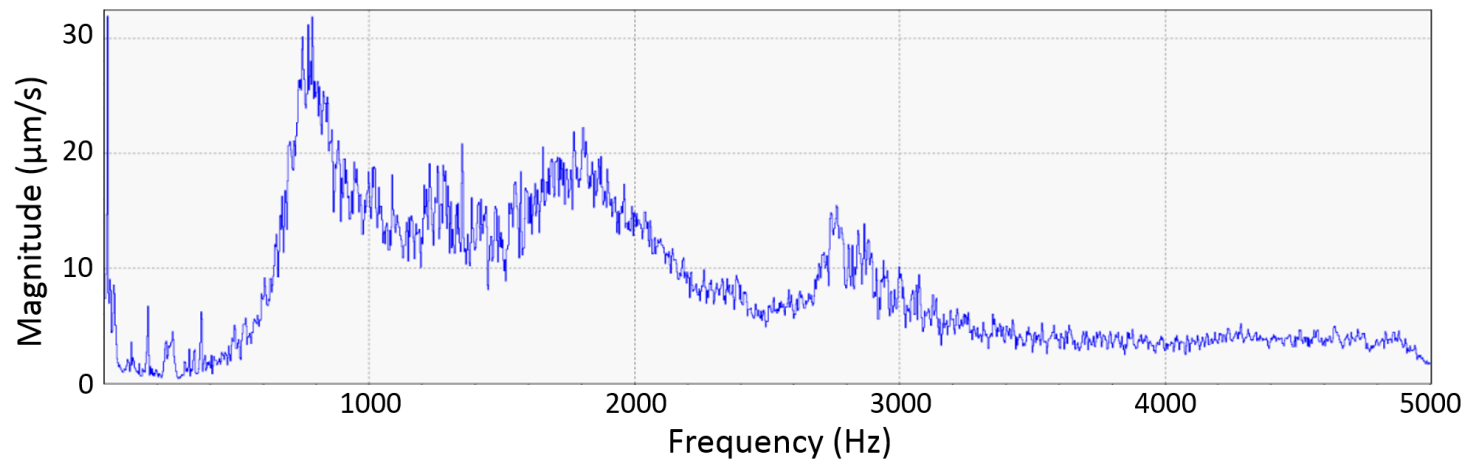

Figure 4.5: This figure shows the frequency spectrum calculated from the SLDV results for the battery electrode with coating 1 in the steel clamp.

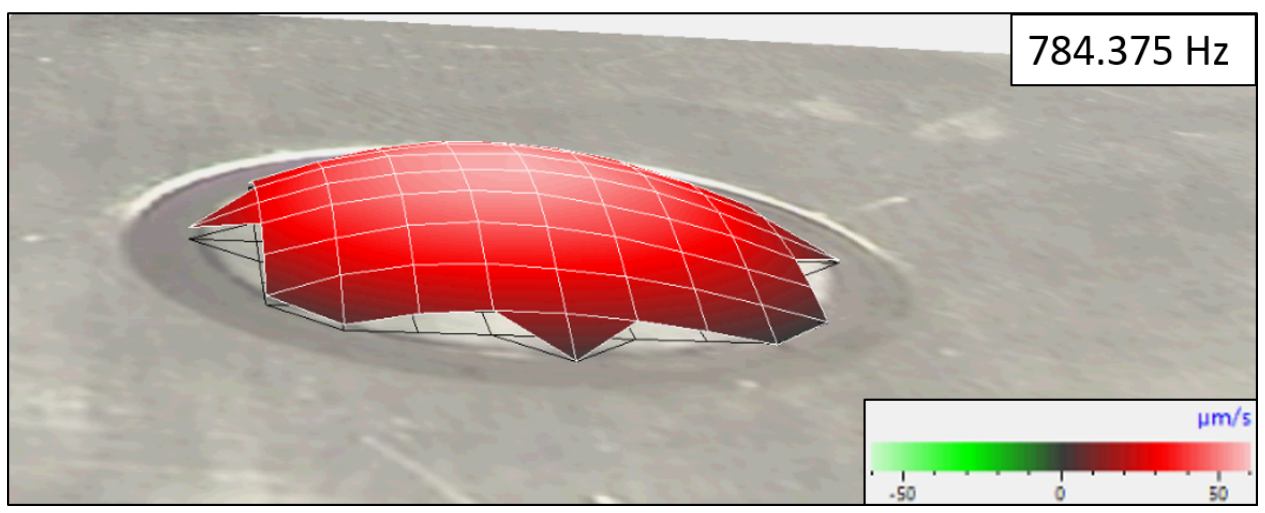

Figure 4.6: This figure shows a velocity map of the battery electrode with coating 1 in the steel clamp at the first frequency peak in Fig. 4.5. The peak occurs around $784 \mathrm{~Hz}$, and from the map here, it looks like a fundamental mode (mode 1 from Fig. 2.1).

and the only peak seen in the frequency spectrum in Fig. 4.15 is significantly higher in amplitude and much narrower than the peaks for the tests done with the steel clamp. The map corresponding to this peak looks like a well-centered fundamental mode (see Fig. 4.16).

\subsection{Drying Tests}

We also found that the resonance frequency changes with other factors, such as drying. Preliminary results were taken by putting paint on a piece of aluminum foil, which was clamped in acrylic clamp in the setup shown in Fig. 3.7, with a speaker as the excitation source. In Fig. 4.17, the resonance peaks are shown for the foil without paint and after several paint applications. The 


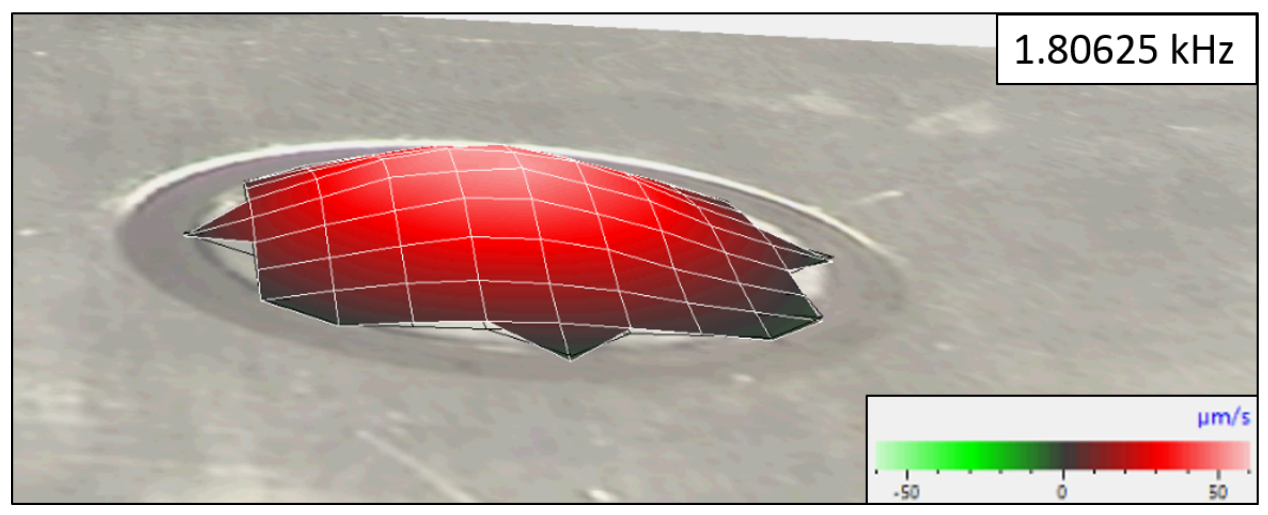

Figure 4.7: This figure shows a velocity map of the battery electrode with coating 1 in the steel clamp at the second frequency peak in Fig. 4.5. The peak occurs around $1806 \mathrm{~Hz}$, and from the map here, it looks similar to the fundamental mode in Fig. 4.6. However, we saw this peak in every material we tried with the steel clamp, so we believe it is an artifact of the clamping system.

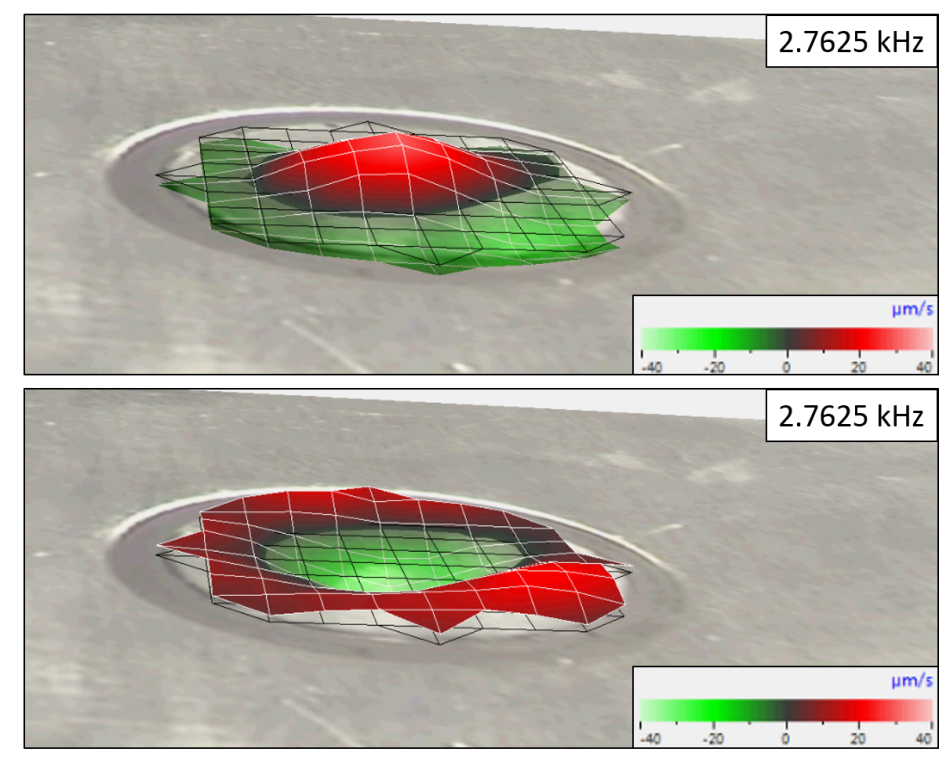

Figure 4.8: This figure shows a velocity map of the battery electrode with coating 1 in the steel clamp at the third frequency peak in Fig. 4.5. The peak occurs around $2763 \mathrm{~Hz}$, and it looks like the second symmetric mode (mode 6 from Fig. 2.1). Two views of this map are shown to illustrate the movement of the film in this mode.

foil was painted with a thin layer of paint and a resonance test was taken once the paint dried. This process was repeated several times without removing the foil from the clamp. The resonance frequency shifts to lower frequencies as more paint is added to the foil. The results in Fig. 4.18 show the tests taken as the second layer of paint dried. Here the resonance actually increases as 


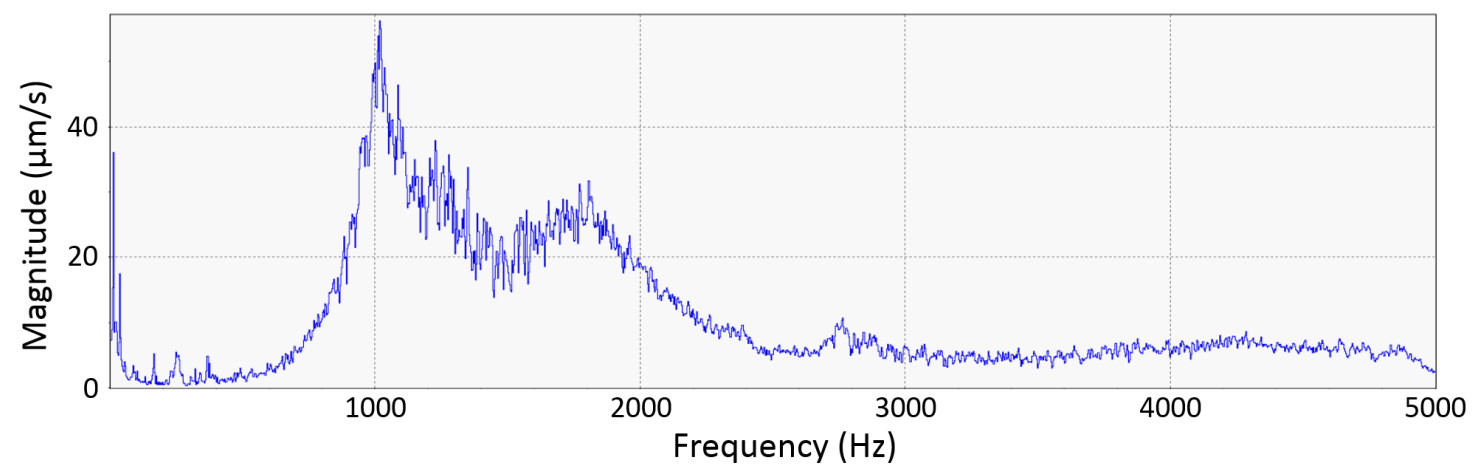

Figure 4.9: This figure shows the frequency spectrum calculated from the SLDV results for the battery electrode with coating 2 in the steel clamp.

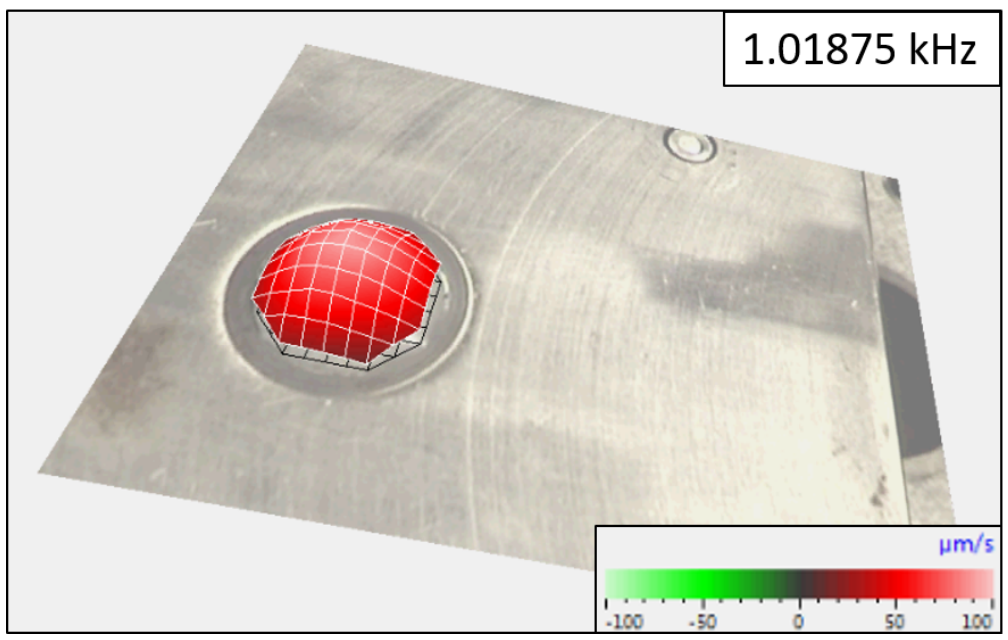

Figure 4.10: This figure shows a velocity map of the battery electrode with coating 2 in the steel clamp at the first frequency peak in Fig. 4.9. The peak occurs around $1019 \mathrm{~Hz}$, and from the map here, it looks like a fundamental mode (mode 1 from Fig. 2.1).

the paint dries, even though there is an overall decrease in frequency from the foil with only one layer of paint.

The drying effect was tested further by putting a drop of acrylic paint (Apple Barrel brand matte acrylic paint) onto aluminum foil while it was in the steel clamp, with the pulsed laser as the excitation source. By taking resonance tests before, after, and as the paint dried, we monitored how the resonance frequency changed over time. Each of the data sets is the average of 200 tests.

The results in Figs. 4.19 and 4.20 were obtained by taking measurements on aluminum foil in the steel clamp setup (Fig. 3.6) with weights (63.0 g) on each end of the foil to apply tension 


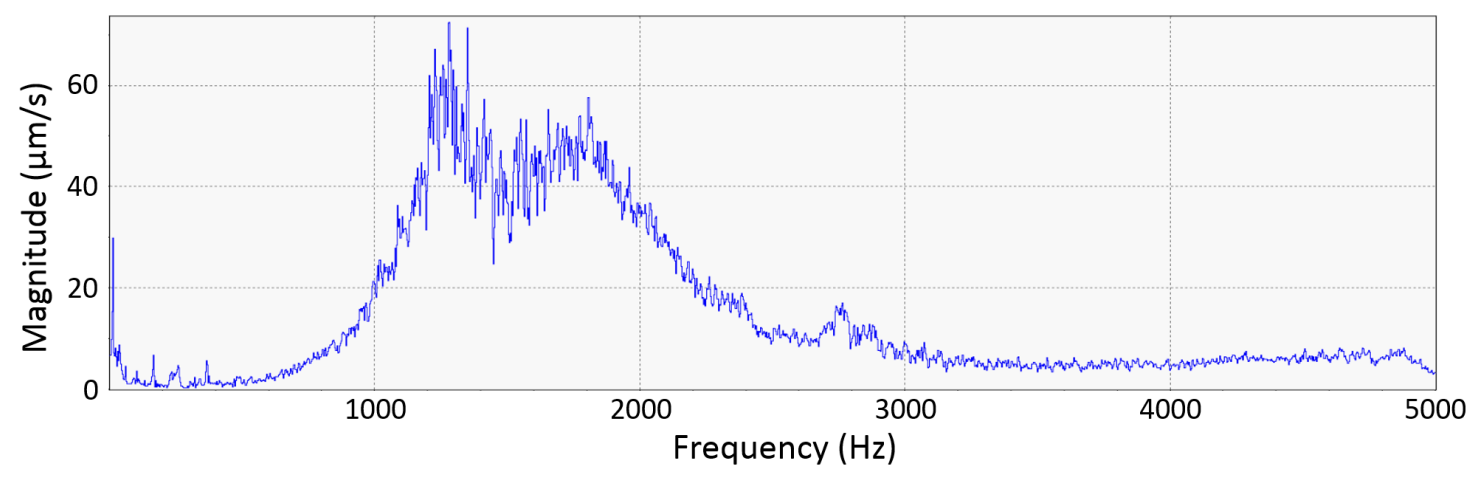

Figure 4.11: This figure shows the frequency spectrum calculated from the SLDV results for the battery electrode with coating 3 in the steel clamp.

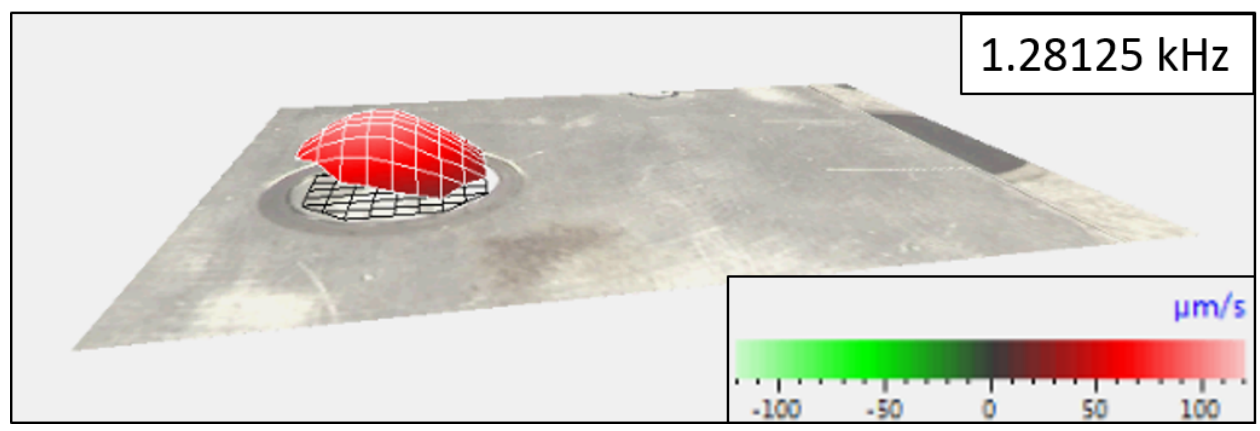

Figure 4.12: This figure shows a velocity map of the battery electrode with coating 3 in the steel clamp at the first frequency peak in Fig. 4.11. The peak occurs around $1281 \mathrm{~Hz}$, and it looks like a fundamental mode (mode 1 from Fig. 2.1).

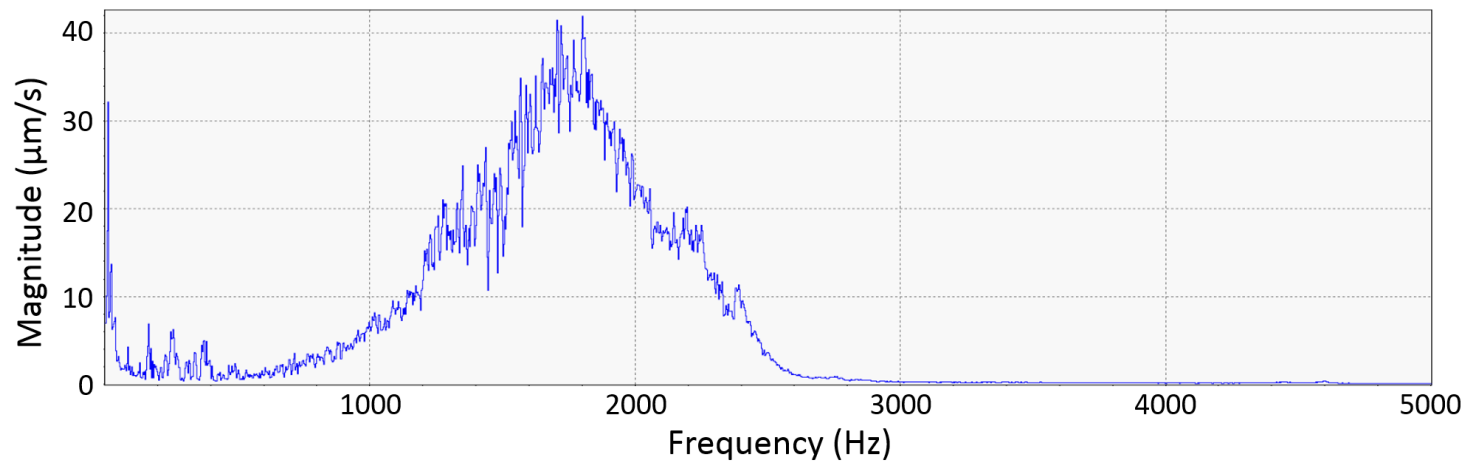

Figure 4.13: This figure shows the frequency spectrum calculated from the SLDV results for a piece of notebook paper with reflective tape. 


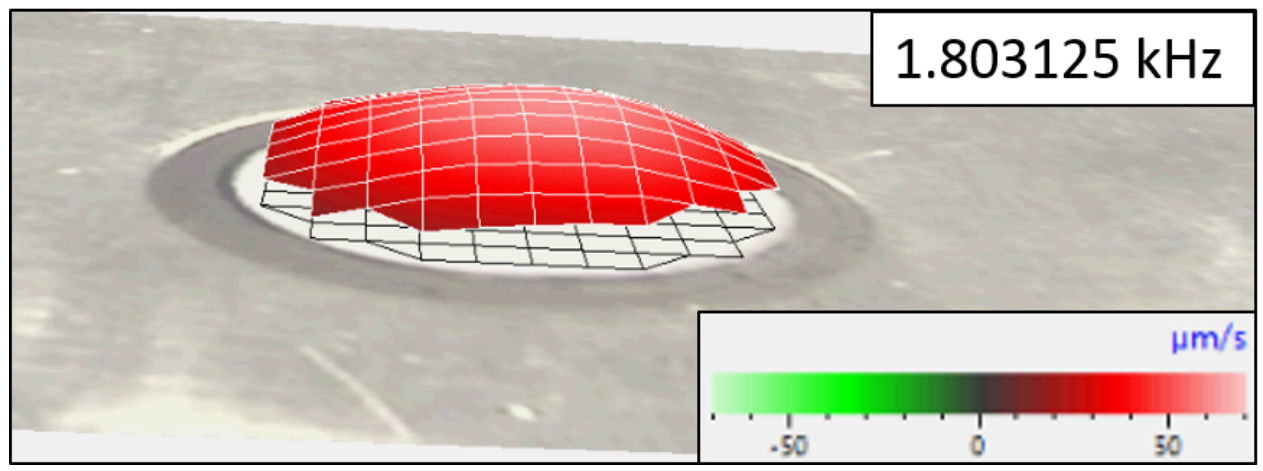

Figure 4.14: This figure shows a velocity map of the notebook paper with reflective tape in the steel clamp at the frequency peak in Fig. 4.13. The peak occurs around $1803 \mathrm{~Hz}$, and it looks like a fundamental mode, like the similar peaks seen in the spectra for the battery electrodes clamped in the steel clamp.

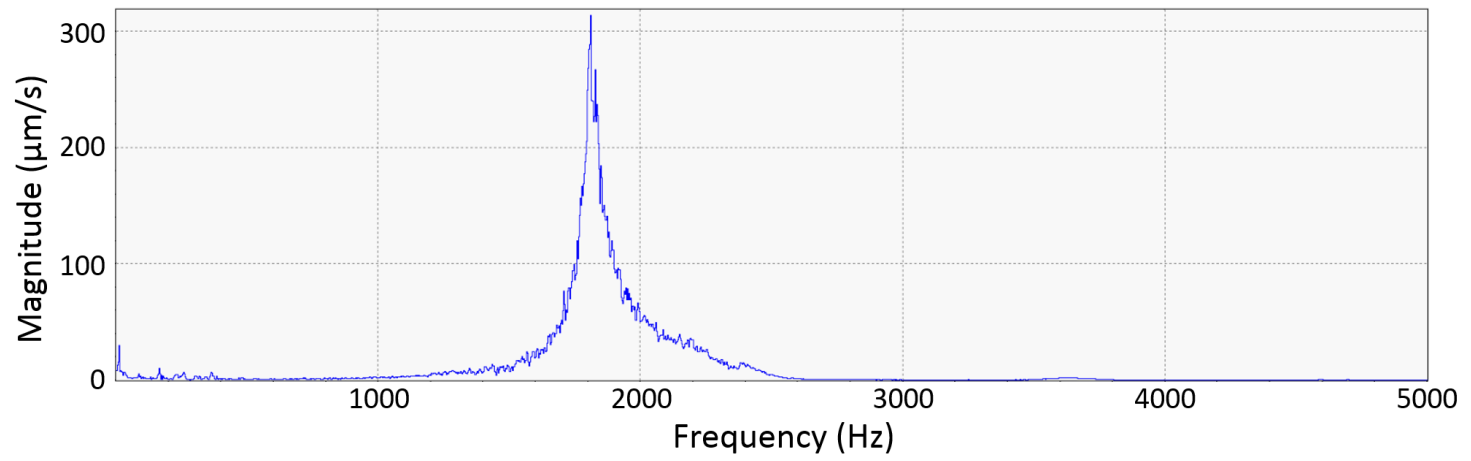

Figure 4.15: This figure shows the frequency spectrum calculated from the SLDV results for a film in the acrylic clamp. The peak here is much narrower and higher in amplitude than the peaks for the films in the steel clamp.

and weights (1253.6 g) on top to help with clamping. These figures show the shift in the resonance frequency as the drop of paint dried. In this test, the drop of paint was diluted with de-ionized water, so the mass may not have been the same as for the other test. The markers in Fig. 4.20 show the peaks identified from Fig. 4.19. The measurements were taken at uneven time intervals as the paint dried. When the paint was first applied, two clear peaks were visible in the acoustic response spectra, around 500 and $1200 \mathrm{~Hz}$. As the paint dried, however, the second (higher frequency) peak decreased and eventually disappeared. Both resonance frequencies increase as the paint dries, with the main peak eventually reaching about the same frequency it was at before the paint was applied (shown by the dashed line in Fig. 4.19 and the filled circle in Fig. 4.20). 


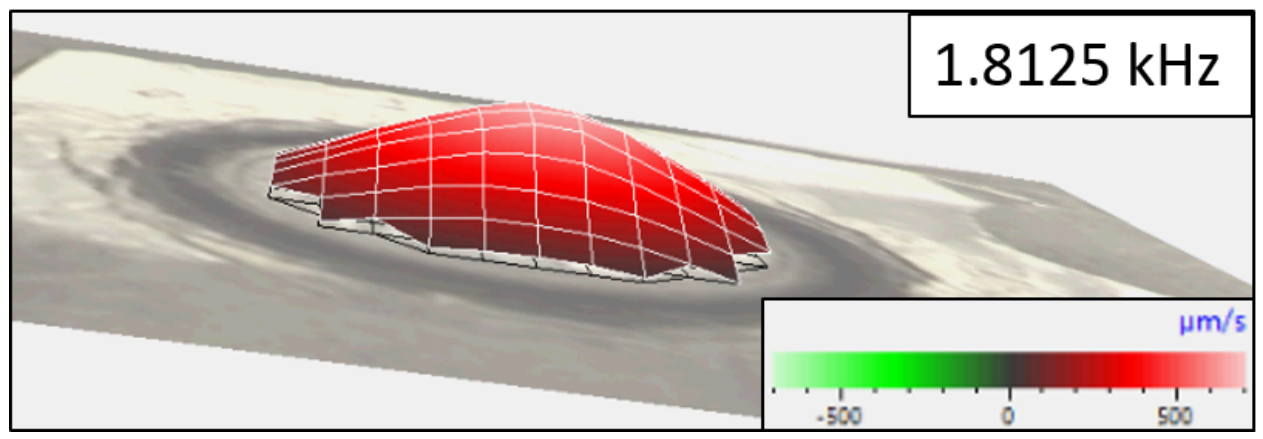

Figure 4.16: This figure shows a velocity map of the battery electrode in the acrylic clamp at the frequency peak in Fig. 4.15. The peak occurs around $1813 \mathrm{~Hz}$, and it looks like a fundamental mode (mode 1 from Fig. 2.1).

Table 4.2: Frequency peaks for each of the three battery cathodes tested. Listed here are the peaks from the SLDV results shown in Figs. 4.5, 4.9, and 4.11 and from the laser excitation test results shown in Figs. 4.3 and 4.4.

\begin{tabular}{lcccc}
\hline \hline Material & $h(\mu \mathrm{m})$ & $\begin{array}{c}\text { peak, SLDV } \\
\text { steel clamp }\end{array}$ & $\begin{array}{c}\text { peak, laser } \\
\text { steel clamp }\end{array}$ & $\begin{array}{c}\text { peak, laser } \\
\text { acrylic clamp }\end{array}$ \\
\hline Coating 1 & 42 & $784 \mathrm{~Hz}$ & $803 \mathrm{~Hz}$ & $1573 \mathrm{~Hz}$ \\
Coating 2 & 38 & $1019 \mathrm{~Hz}$ & $1066 \mathrm{~Hz}$ & $1873 \mathrm{~Hz}$ \\
Coating 3 & 26 & $1281 \mathrm{~Hz}$ & $1211 \mathrm{~Hz}$ & $2289 \mathrm{~Hz}$ \\
\hline \hline
\end{tabular}

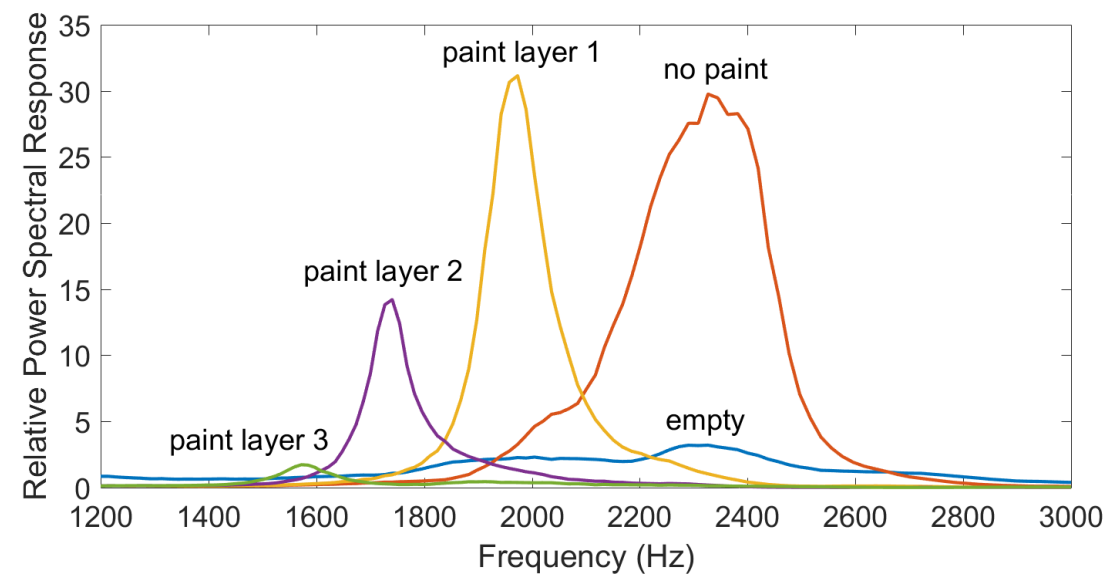

Figure 4.17: The shift in frequency peaks for an aluminum film with multiple thin, dried layers of paint. The film was clamped in the acrylic clamp as shown in Fig. 3.7. Speaker sweep tests were taken before paint was applied and after each additional thin layer of paint dried. A test was also taken without the aluminum in the clamp ("empty") for a baseline. The peaks shift to lower frequencies as more paint is applied. 


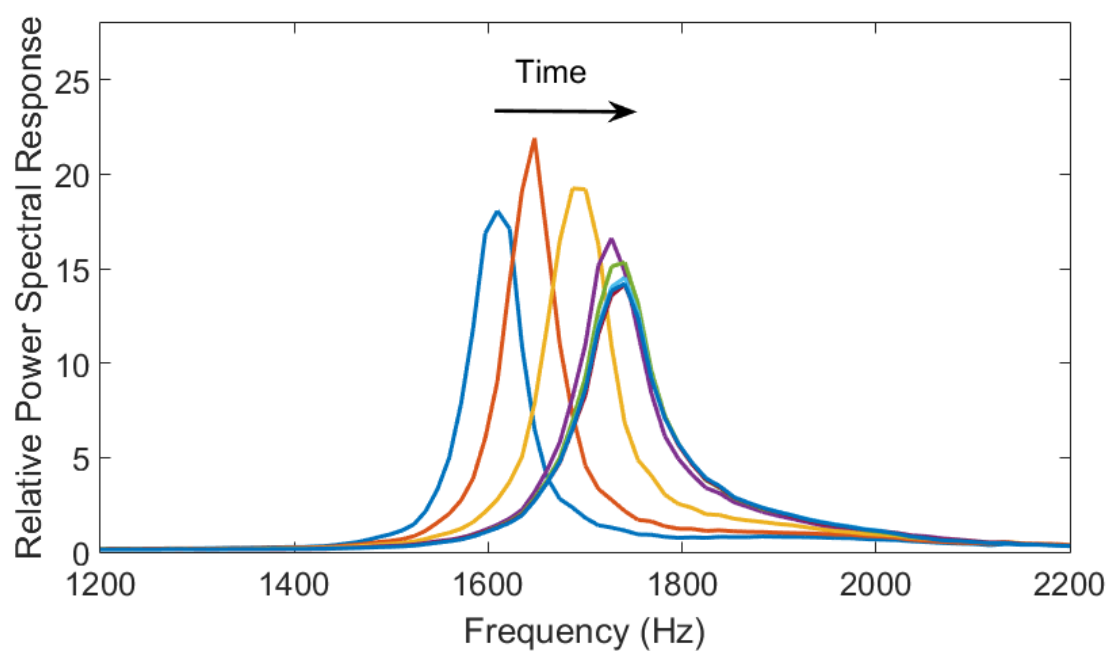

Figure 4.18: The shift in frequency peaks for an aluminum film with a drying, thin layer of paint. The film was clamped in the acrylic clamp as shown in Fig. 3.7. Speaker sweeps were taken as the second layer of paint in Fig. 4.17 dried. The peaks shift to higher frequencies as the paint dries, as indicated by the arrow.

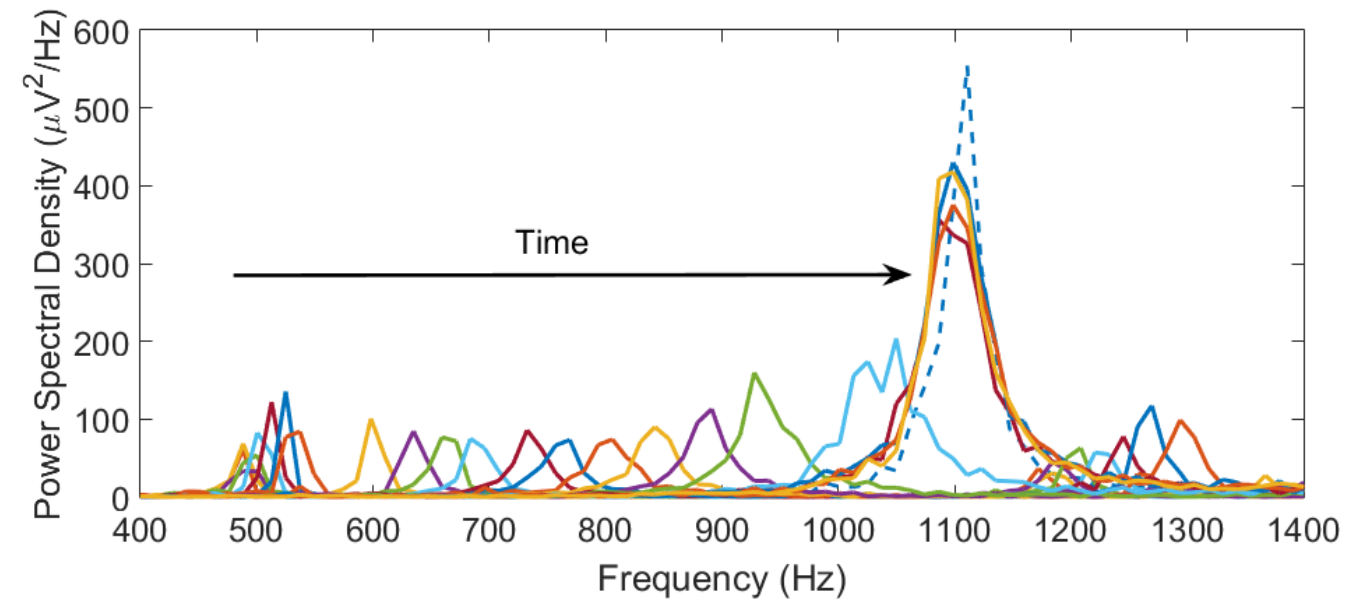

Figure 4.19: The shift in frequency peaks for an aluminum film with a drying layer of diluted paint. The peaks were identified from the acoustic response spectra of the film, clamped as shown in Fig. 3.6 with weights $(63.0 \mathrm{~g})$ on each end of the aluminum strip to apply tension. Resonance tests were taken before paint was applied (dashed line) and as the paint dried. The peaks shift to higher frequencies as the paint dries, as indicated by the arrow.

The results in Figs. 4.21 and 4.22 show a repeated test with a drop of undiluted paint (weighing $28.0 \mathrm{~g}$ ) on an aluminum film. This film was about $23 \mu \mathrm{m}$ thick and, without paint, had a resonance of about $1200 \mathrm{~Hz}$ (shown by the dashed line in Fig. 4.21 and the filled circle in 


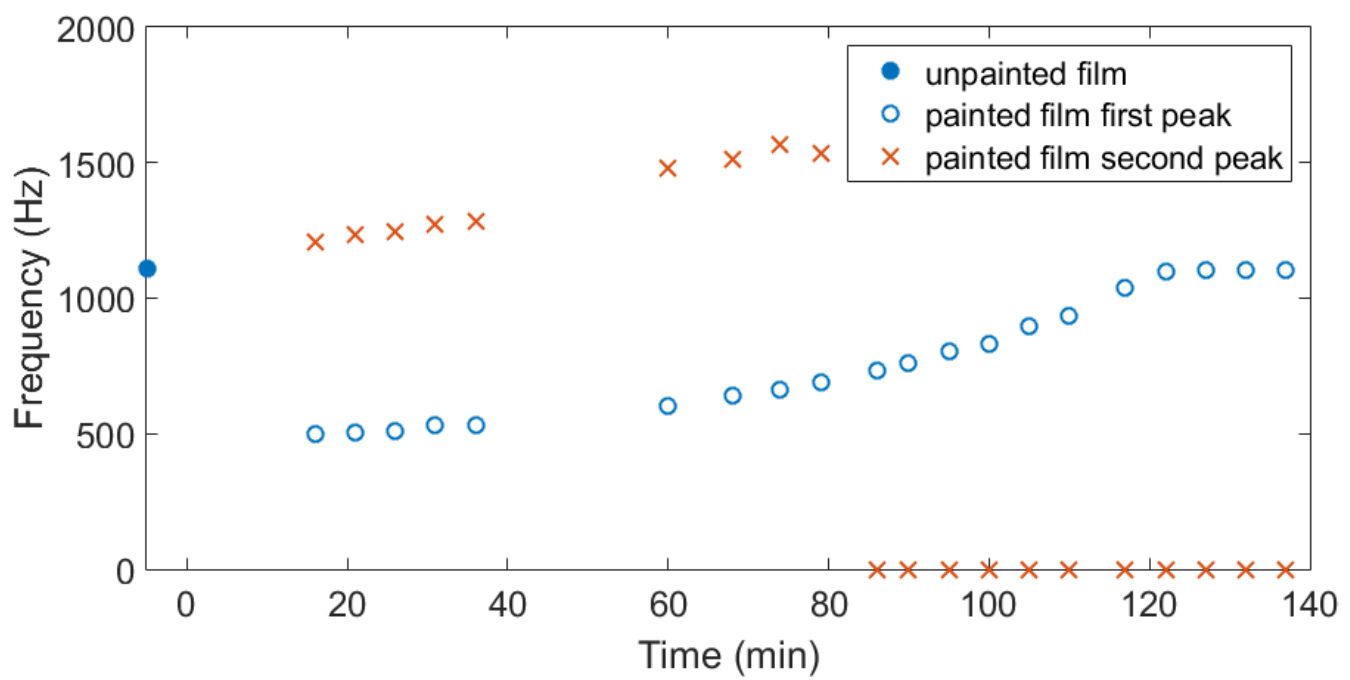

Figure 4.20: The shift in frequency peaks for an aluminum film with a drying layer of diluted paint. The peaks were identified from the acoustic response spectra of the film seen in Fig. 4.19. Resonance tests were taken before paint was applied (filled circle) and as the paint dried. Two clear peaks were present initially, but the second peak (red x) disappeared as the paint dried (shown as zero values on the graph). Both peaks shift to higher frequencies as the paint dries. Measurements were taken at uneven time intervals, so the points are not spaced evenly over time.

Fig. 4.22). For this set of tests, there were no weights to apply tension on the foil, but there were weights $(1253.6 \mathrm{~g})$ on top of the clamp to help provide a more fixed boundary. The magnitude of the results here is much lower than that of the previous test, making the peaks more difficult to distinguish from the noise. Because the signal is noisier, only results from every 20 minutes are shown in Fig. 4.21 for clarity, while Fig. 4.22 shows where the peak occurred for all of the tests, which were taken every 5 minutes. Since the results were noisy and did not always have a single clear peak, the peak values shown in Fig. 4.22 were found by fitting a Gaussian curve to the power spectral density obtained from the microphone data.

The results presented here and their implications are discussed in the next chapter. 


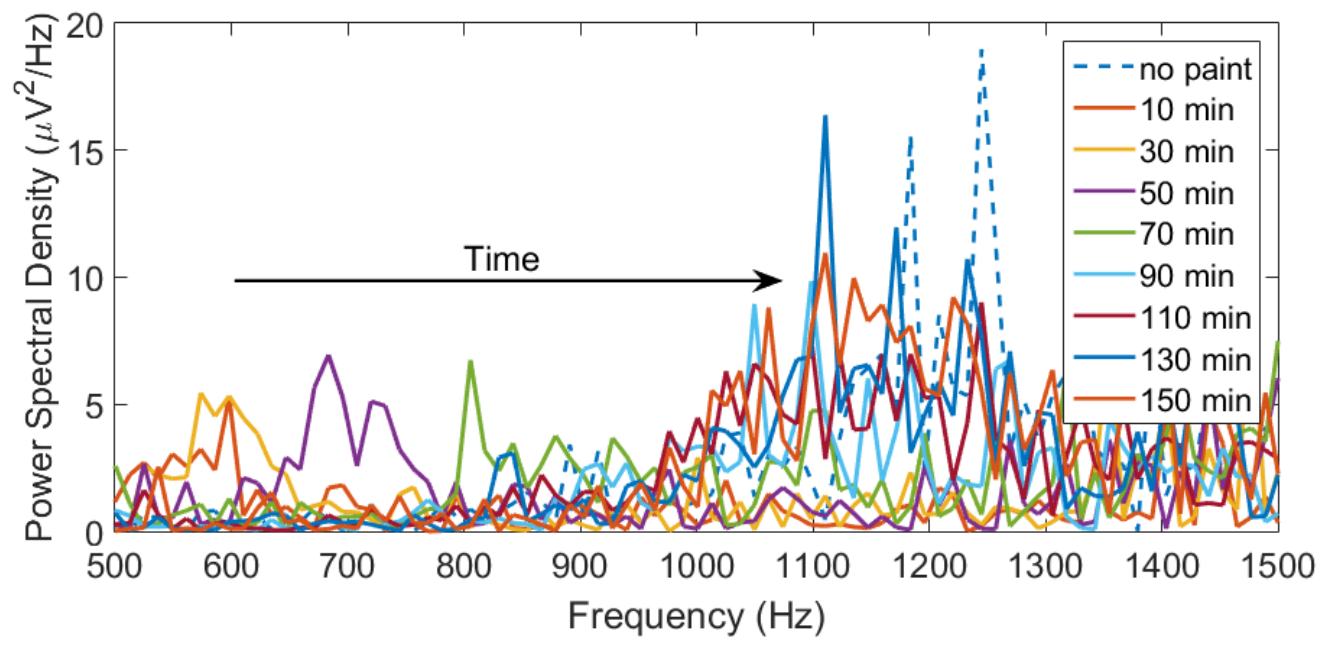

Figure 4.21: The shift in frequency peaks for an aluminum film with a drying layer of paint. The peaks were identified from the acoustic response spectra of the film, clamped as shown in Fig. 3.6. Resonance tests were taken before paint was applied (dashed line) and every 5 minutes as the paint dried. In order to better distinguish peaks, only the results from tests taken every 20 minutes are shown in this graph. The peaks shift to higher frequencies as the paint dries, as indicated by the arrow, with the largest shift occurring between 70 and $90 \mathrm{~min}$.

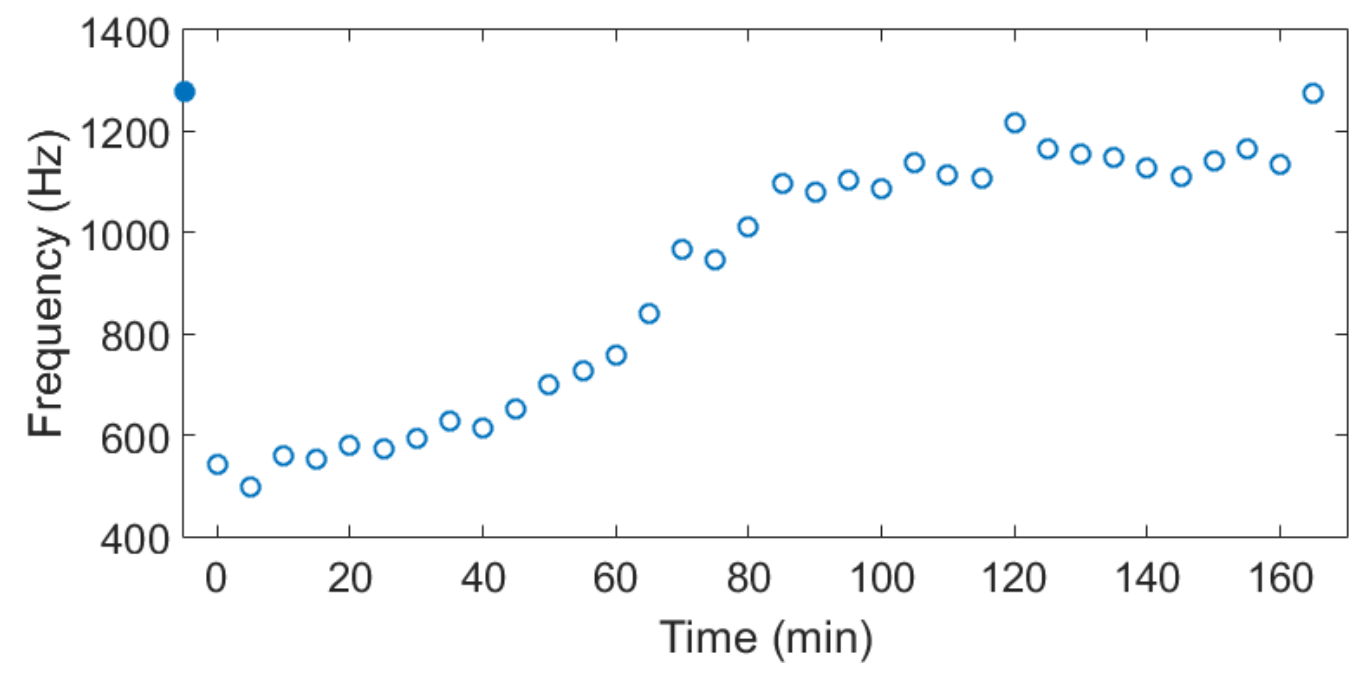

Figure 4.22: The shift in frequency peaks for an aluminum film with a drying layer of paint. The peaks were identified from the acoustic response spectra of the film seen in Fig. 4.21. Resonance tests were taken before paint was applied (filled circle) and every 5 minutes as the paint dried. The peak shifts to higher frequencies as the paint dries. 


\section{CHAPTER 5. DISCUSSION}

This chapter contains a discussion of the results presented in the previous chapter. Although the results are promising, there are still some issues with repeatability. This may be largely due to the clamping not being as consistent as we had hoped. Non-uniformity of the battery films may also be a factor.

\subsection{Thin Plate/Membrane Behavior in Results}

Using the setup shown in Fig. 3.6 with either clamp resulted in resonance tests that were able to distinguish between different commercial-grade battery films. Our experimental results appear to validate our simulations. From the simulations shown in Chapter 2, we expected thicker metal films to have higher resonance frequencies, even though this trend is opposite the expected results for a thicker battery film. The simulations showed that the aluminum film acted more like a plate, with $h$ (thickness) dependence, while the battery film acted more like a membrane, with

$\sqrt{1 / h}$ dependence. In the case of the aluminum films, a thickness increase also causes an increase in rigidity, which influences the frequency shift more than the resulting mass increase, since a thicker plate has a higher resonance frequency. The variation in battery film thickness was only in the coating thickness, which has a much lower stiffness than the aluminum current collector. This likely made the effect more of a mass-loading effect, rather than a change in rigidity, since an increase in mass would be expected to produce a decrease in frequency. Two sets of simulations support this hypothesis. The first set of simulations, shown by the solid line in Fig. 5.1, holds the density of the coating layer constant while varying the coating thickness. The second set, shown by the dashed line in the same figure, varies the coating density but holds the coating thickness constant. The thickness and density were varied so that the mass of the coating was identical in both sets. Both sets give very similar results and follow the trend of the experimental results for 


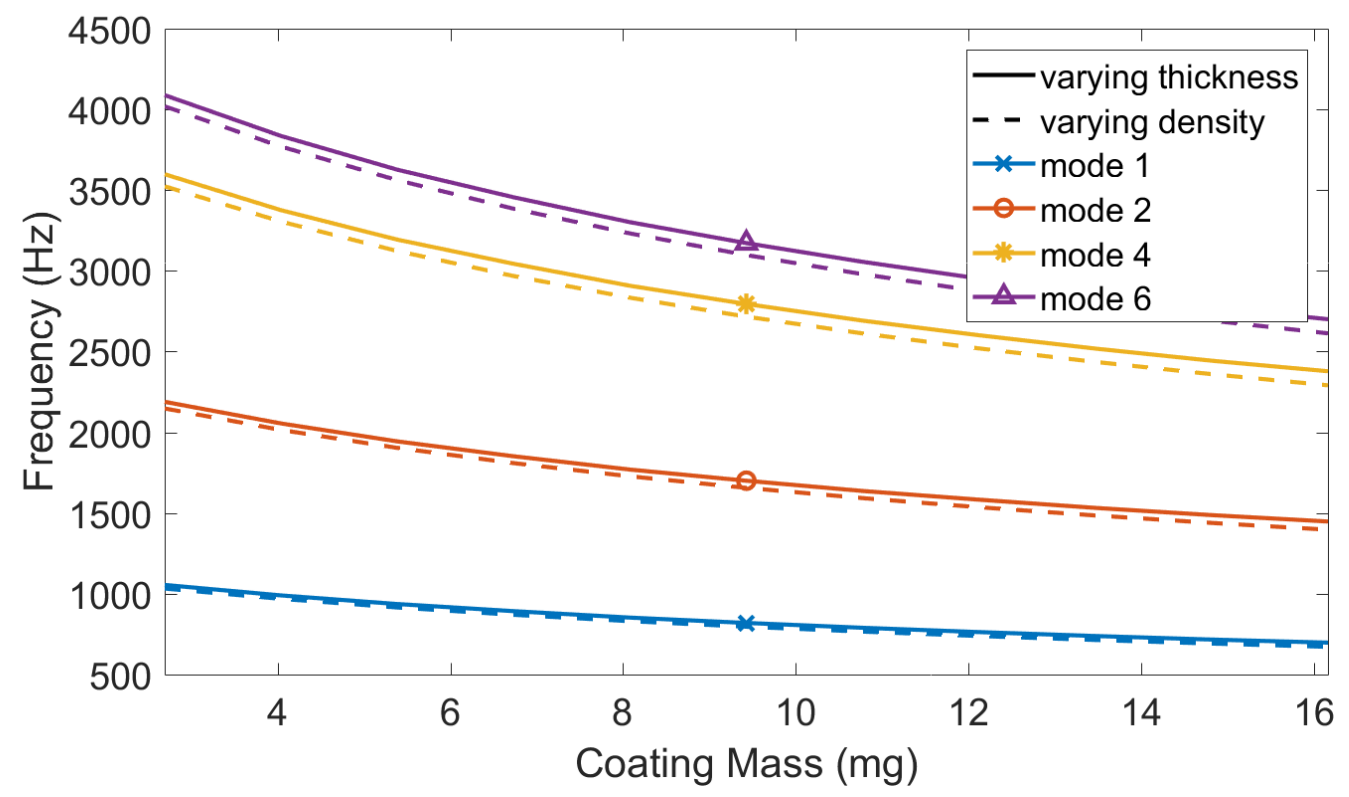

Figure 5.1: Simulations of battery cathode with properties listed in Table 2.4. The solid lines show the resonance frequencies for four modes with a varying coating thickness and constant density $\left(2.029 \mathrm{~g} / \mathrm{cm}^{3}\right)$, while the dashed lines show the frequencies for a constant coating thickness (30 $\mu \mathrm{m})$ and varying coating density. The density was varied to give the same mass as the coating with varying thickness, so that the mass variations were the same for both data sets.

battery films of different thicknesses. This suggests that the frequency shift caused by coating thickness changes in the battery electrodes is mainly due to mass differences.

We also found that the cathodes used in the tests for Figs. 4.3 and 4.4 had coatings with different densities. The thickness and density values are listed in Table 5.1. The density values in this table were calculated based on the reported thickness of the film coating and current collectors, and comparison with Table 4.1 shows that this can cause a significant difference in the resulting density. It should be noted that some of the density variation is likely due to inexact surface area and thickness measurements. This variation in density should also have some effect on the resonance frequency, as seen in Fig. 2.5, and could account for why the frequency shift seen in Figs. 4.3 and 4.4 between the coatings with a $4 \mu \mathrm{m}$ difference is similar to the shift between those with a $12 \mu \mathrm{m}$ thickness difference. From our analytical model in Eq. (2.4), we expect the thicker (and therefore more massive) films to have a lower resonance, and this is the case with both the film simulation shown in Fig. 2.4 and the experimental results in Chapter 4. 
Use of the two different clamps resulted in very different resonance peaks for the same battery films. The frequency peaks seen in Fig. 4.3, taken with the films in the steel clamp, are broader, noisier, and at lower frequencies than those in Fig. 4.4, which were taken with the acrylic clamp. The difference between the peaks for the same film in each clamp ranges from about $600 \mathrm{~Hz}$ to $1 \mathrm{kHz}$. When the films were removed from the acrylic clamp, there was visible deformation from where the o-rings were in contact with the films. One possible reason is that as the acrylic clamp was tightened and the o-rings compressed, the films were stretched some amount and tension was applied to the films. From Eq. (2.4), we see that, for a membrane, the frequency is an increasing function with respect to the tension. Since the simulations in Chapter 2 showed that the battery films act more like a membrane, we would expect the electrodes to follow this trend of increasing frequency with tension. Although the larger vibration area for the acrylic clamp would cause a decrease in frequency, since the tension was large enough to ultimately stretch the cathodes, the tension dominated the frequency change. The peaks may be narrower and more well-defined than the peaks from the steel clamp due to more effective and uniform edge confinement. This led to a trade-off: the acrylic clamp gave cleaner results and a well-clamped boundary, but it noticeably deformed the films. Use of the steel clamp did not result in visible change of the films, but the broader, noisier peaks indicate that the boundary may not be as fixed as we would prefer. The SLDV results seem to confirm this, as the map of the film movement for the fundamental mode for some of the films, especially in Fig. 4.12, show that the vibration pattern is not centered in the exposed film area. This lack of symmetry could be due to variations in the battery film itself, but since it was present for all three films (though not visible in all of the frames shown in Chapter 4) and not for the acrylic clamp, it is more likely because of poor clamping. A few tests with the SLDV seem to support this theory, as rotating the whole clamp (without removing the film) resulted in the asymmetry rotating as well, indicating that the asymmetry was not due to the acoustics of the setup. Rotating the film in the clamp resulted in the same pattern (no shape rotation), which suggests that the clamp itself is the main source of the observed asymmetry.

Aside from imperfections in the clamping, the clamps themselves could also act as thin plates which are excited into resonance and influence the frequency response through the physical contact with the vibrating film. Since the two clamps were made of very different materials, the steel and acrylic likely influenced the resonance differently. This could contribute to the disparity in 
resonance frequencies for the same film in each clamp and was not accounted for in the numerical model, which only included a perfectly fixed boundary.

Another possible reason for the discrepancy between the resonance frequencies obtained with the two clamps is the vibration area being different than the measured clamp dimensions. The measured radius of the confined film was $0.64 \mathrm{~cm}$ for the steel clamp and $0.706 \mathrm{~cm}$ for the acrylic clamp, which would indicate that the acrylic clamp should have a lower frequency for the same film. Figure 5.2 shows the results of a simulation of a battery cathode with properties listed in Table 2.4 at various radii, and Fig. 5.3 shows the results of the same simulation for a battery cathode with "Coating 1" in Table 5.1. The frequency shift seen here is not as large as that seen in the previous chapter between the two clamps, and the results show that the acrylic clamp should have the lower resonance frequency, indicating that this area difference is not the only cause. These figures show that for a shift of $1 \mathrm{kHz}$ in mode 1 , a radius difference of greater than $0.1 \mathrm{~cm}$ is required. Tightening the acrylic clamp to different amounts results in a smaller area as the o-rings are compressed more, so the radius for the acrylic clamp tests may have been slightly different than the measured value, but should not have been large enough to account for the frequency shift. However, it is also possible that the vibration area for the steel clamp is larger than the hole in the clamp, since the SLDV results suggest that the boundary is not evenly confining the edge of the film. This would make the steel clamp radius larger than the measured $0.64 \mathrm{~cm}$ by an unknown amount, resulting in a lower frequency.

Table 5.1: Measured and calculated properties of the battery films. The thickness is the reported value from the manufacturers (of the coating only - not including the $20-\mu \mathrm{m}$-thick aluminum current collector), the density is the calculated value, the Poisson's ratio is assumed [42], and the Young's modulus values are obtained from a combination of simulated and experimental results (discussed below).

\begin{tabular}{lccccc}
\hline \hline Material & $h(\mu \mathrm{m})$ & $\rho\left(\mathrm{g} / \mathrm{cm}^{3}\right)$ & $E(\mathrm{GPa})$, SLDV & $E(\mathrm{GPa})$, peaks & $v$ \\
\hline Coating 1 & 42 & 2.760 & 0.344 & 0.412 & 0.3 \\
Coating 2 & 38 & 3.055 & 1.71 & 2.01 & 0.3 \\
Coating 3 & 26 & 2.479 & 5.04 & 4.13 & 0.3 \\
\hline \hline
\end{tabular}

The resonance peaks obtained from the measurements in either clamp are not very consistent. By repeating the measurements taken on the three types of battery electrode films, it is 


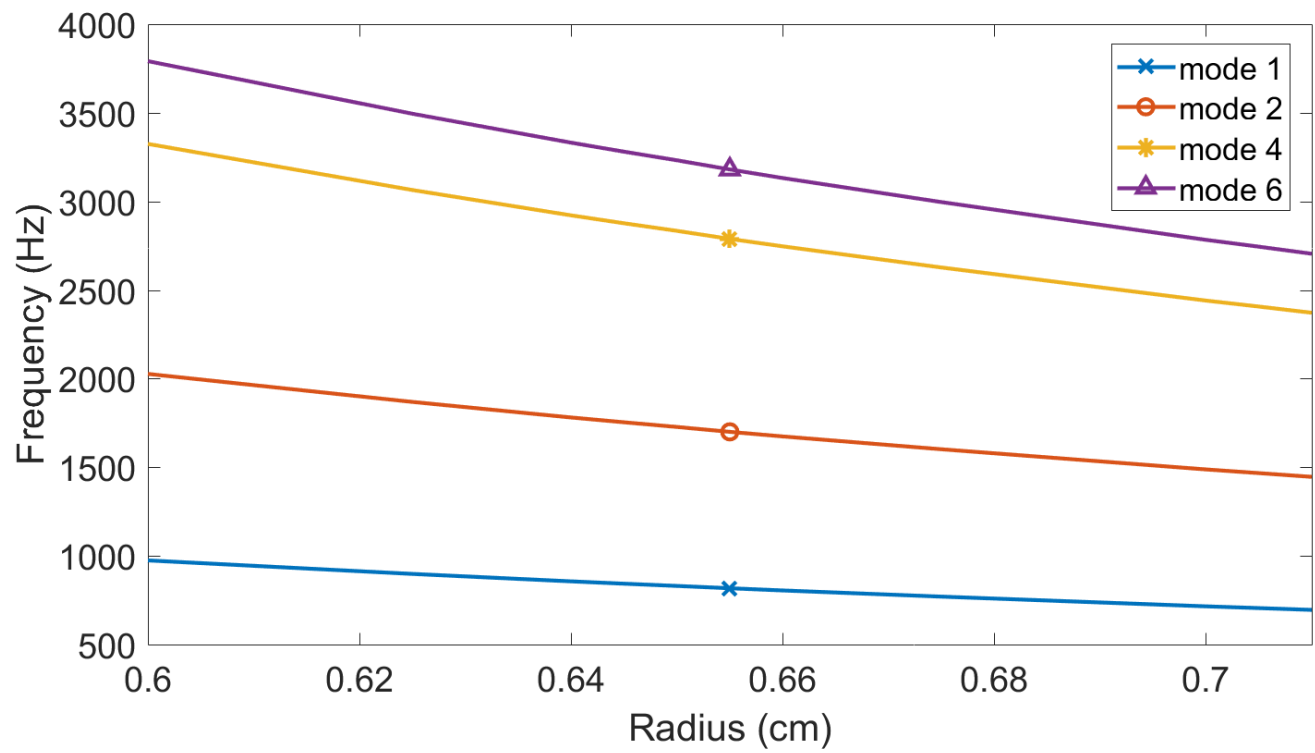

Figure 5.2: Simulations of battery cathode with properties listed in Table 2.4 and a varying radius. This shows how changing the area of the vibration affects the resonance frequencies. The difference in area between the acrylic clamp with compressed o-rings and the steel clamp does not cause a frequency shift as large as that seen in the results in the previous chapter.

possible to obtain averages and $95 \%$ confidence intervals for the resonance frequencies. These statistics for tests done with the films in the steel clamp are listed in Table 5.2, and those for tests done with the acrylic clamp are listed in Table 5.3. Since some of the results were noisy and did not have one clear peak, especially those for the steel clamp, the values for the peaks were found by fitting a Gaussian curve to the power spectral density obtained from the microphone data, such as those shown in Figs. 4.3 and 4.4. The confidence intervals for some of the cathodes overlap, and the range is significant (about 200-400 Hz). Some of the non-repeatability could be due to wrinkles or non-uniformities in the battery films themselves, but some is likely caused by differences in clamping.

Our efforts to consistently and securely clamp the edges of the film caused a calendering effect outside of the area of interrogation. The material in the film coating was being compressed by the clamp, resulting in a change in the physical properties. Li-ion films are calendered during the manufacturing process in order to achieve a specific porosity and thickness [11]. This porosity affects the electronic properties of the battery and changes the density of the overall film. When we changed the porosity with our clamping, it did not change the properties of the part of the film 


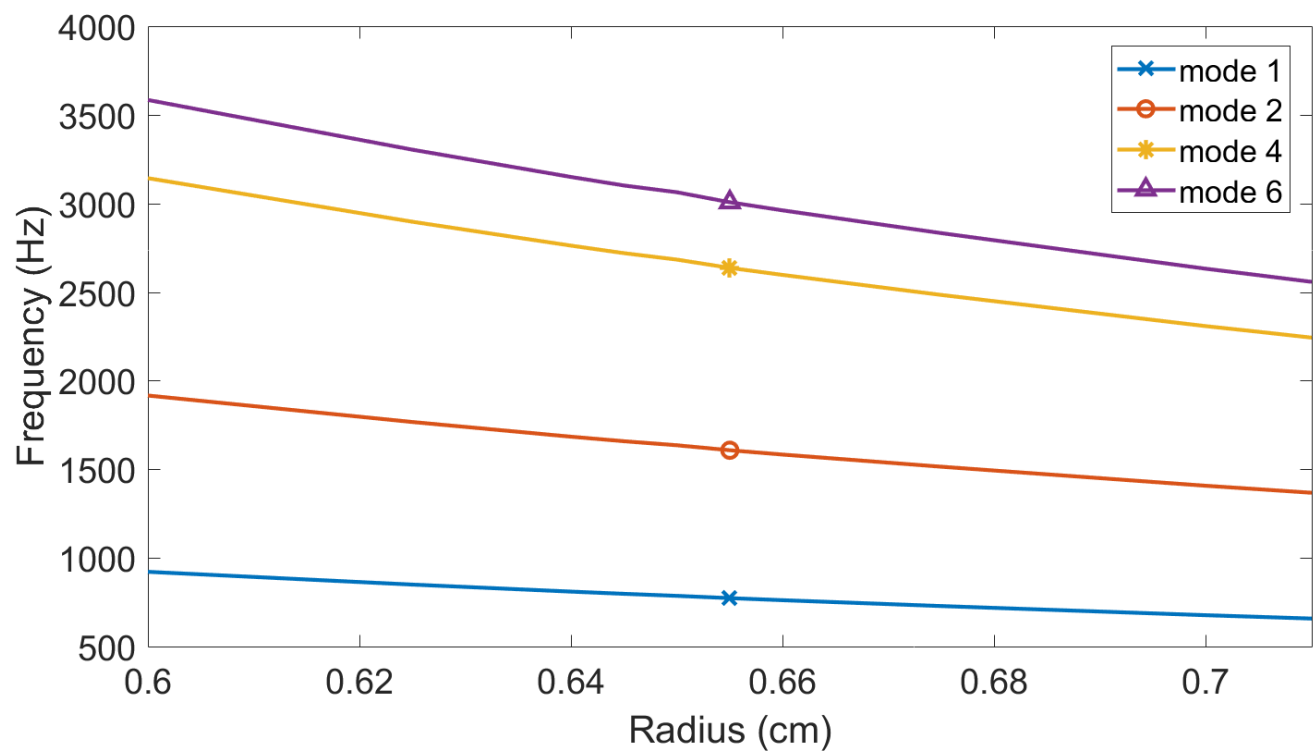

Figure 5.3: Simulations of battery cathode with properties listed under "Coating 1" in Table 5.1 and a varying radius. This shows how changing the area of the vibration affects the resonance frequencies. The difference in area between the acrylic clamp with compressed o-rings and the steel clamp does not cause a frequency shift as large as that seen in the results in the previous chapter.

that was currently being measured (as that was the area within the confinement), but it meant that we could not repeat measurements in the same place on our film. We are currently experimenting with other techniques for this experiment that do not calender the edges of the electrode.

A comparison of the results for the two drying tests performed with laser excitation shows that there is a very large magnitude difference in the power spectral density. The significantly lower magnitude in Fig. 4.21 gives a lower signal-to-noise ratio, which makes the resonance peaks less prominent. There were two main differences between the measurement setups: the first test used a drop of paint diluted with water and had weights on the ends of the aluminum strip to apply tension, while the second test had a drop of undiluted paint and no tension weights. However, it seems unlikely that either of these caused such a large change in magnitude.

The drying tests show an interesting interplay between changing mass and stiffness. When paint is first applied, it mainly acts like an added mass and decreases the resonance frequency by a large amount. As the paint dries, material evaporates, lowering the mass-loading and leading to an expected increase in frequency from the wet paint case. The paint also changes the stiffness of the 
Table 5.2: Frequency peaks and statistics of the three battery films that were tested in the steel clamp with laser excitation. The peaks were identified by fitting a Gaussian curve to the spectra, such as the one in Fig. 4.3. The average and $95 \%$ confidence intervals of the peaks are also listed.

\begin{tabular}{lccc}
\hline \hline Material & peak $(\mathrm{Hz})$ & average $(\mathrm{Hz})$ & $\begin{array}{c}\text { 95\% Confidence } \\
\text { Intervals }(\mathrm{Hz})\end{array}$ \\
\hline & 916.7 & & 731.7 \\
Coating 1 & 748.5 & 848.2 & 964.7 \\
& 885.7 & & 990.5 \\
& 841.8 & & \\
Coating 2 & 1040 & & 1153 \\
& 1022 & 1072 & 1047 \\
& 1135 & & \\
Coating 3 & 1090 & & 1410 \\
\hline \hline
\end{tabular}

composite film, further increasing the resonance. We suspect this is the reason that the resonance of the foil with a fully dried paint drop was similar to the resonance of the foil without paint; the stiffness changed, offsetting the decrease in frequency due to the final amount of additional mass. The wet drop of undiluted paint weighed $28.0 \mathrm{~g}$, but the dry paint weighed only $9.7 \mathrm{~g}$, so a significant amount of mass was lost as the paint dried. The analytical model for thin plates (Eq. 2.2), which predicts the behavior of aluminum films quite well, also predicts that additional mass or density will cause the resonance frequency to decrease. However, this particular system with the paint cannot be represented well by the simplified model. Since the drop of paint nearly covered the vibration area of the film, the painted aluminum becomes almost a two-layer system, which the simulations in Chapter 2 show is not approximated well by the thin plate model. There are several other complicating factors, such as varying thickness of the paint layer across the drop, an unknown volume change from the wet drop to dry, and an unknown change in stiffness. For the results shown in Fig. 4.17, the dried paint looks like the mass-loading was the dominant factor, as each layer of paint shifts the frequency to lower values and decreases the amplitude by dampening the response. 
Table 5.3: Frequency peaks and statistics of the three battery films that were tested in the acrylic clamp with laser excitation. The peak values were identified by fitting a Gaussian curve to the spectra, such as the one in Fig. 4.4. The average and 95\% confidence intervals of the peaks are also listed.

\begin{tabular}{lccc}
\hline \hline Material & peak $(\mathrm{Hz})$ & average $(\mathrm{Hz})$ & $\begin{array}{c}\text { 95\% Confidence } \\
\text { Intervals }(\mathrm{Hz})\end{array}$ \\
\hline & 1666 & & 1508 \\
Coating 1 & 1690 & 1697 & 1886 \\
& 1858 & & 1848 \\
& 1573 & & \\
\hline & 2058 & & 2180 \\
Coating 2 & 2118 & 2014 & 2065 \\
& 2006 & & \\
& 1873 & & 2432 \\
Coating 3 & 2260 & & \\
& 2358 & 2249 & \\
\hline \hline
\end{tabular}

Drying tests such as these could have applications for the battery manufacturing process, as it shows that the acoustic response changes as the film dries. This could allow for monitoring of the drying state of the electrode during the drying of the slurry that forms the coating on the current collector [11].

\subsection{Extracting Young's Modulus}

One interesting aspect of our acoustic measurement method is the potential to extract the Young's modulus of the battery coating. By fitting the acoustic measurements to the results of the numerical model, the Young's modulus could be found. Because the numerical model did not include an applied tension, we compare the simulations to the experimental results for the films in the steel clamp, which, unlike the acrylic clamp, does not appear to apply tension to the films.

As mentioned in Chapter 3, we used a scanning laser Doppler vibrometer (SLDV) to ensure that the peaks we were obtaining from our other measurements were actually the fundamental frequencies of the films. The SLDV results confirmed that the peaks in Fig. 4.3 are the fundamental frequencies (mode 1 as seen in Fig. 2.1) of the three cathodes. For the film with a 42- $\mu$ m-thick 
coating, the chirp also excited the second symmetric mode (mode 6 as seen in Fig. 2.1), giving two data points to fit with the simulation results.

In Fig. 5.4, we have the simulated results for the first four unique resonance frequencies for a film with similar properties to the cathode with a coating thickness of $42 \mu \mathrm{m}$ seen in Fig. 4.3, along with the peak values for modes 1 and 6 obtained from the SLDV results for that film (shown by the dashed lines). By weighing the sample and using the reported coating thickness of $42 \mu \mathrm{m}$, we found the coating density was $2.760 \mathrm{~g} / \mathrm{cm}^{3}$. We used an assumed value of 0.3 for the Poisson's ratio [42] and ran simulations with a wide variety of values for the Young's modulus, hoping to be able to fit the measured resonances to the data to find the elasticity of our film. The Young's modulus value given by mode 1 is $344 \mathrm{MPa}$, and the value given by mode 6 is $87.3 \mathrm{MPa}$. While the two modes don't agree, the values are within the same order of magnitude. This difference could be caused by inaccuracy in the numerical model, particularly since the mesh sensitivity analysis showed that the higher modes had larger frequency shifts (though the same percent change) than the lower modes. It could also be due to the relatively low resolution of the SLDV results. Increasing the number of averages or the number of scan points for the SLDV scan may have resulted in a slightly different frequency peak. The result obtained using mode 1 is likely more trustworthy for several reasons. The shift in frequency caused by changing the mesh size is smaller for the lower modes than for the higher ones, so the lower frequency is less sensitive to the simulation mesh size, even though the slope for the lower mode (as seen in Fig. 5.4) makes the extracted Young's modulus more sensitive to frequency changes than the higher mode. Furthermore, there is less energy in the higher modes, since the first mode is preferentially excited [40]. The fundamental mode is also more efficient at producing sound than the higher modes [42]. This is seen in the frequency content for the films, where the first mode has the largest peak. Additionally, at higher frequencies, such as those correlated with mode 6 , the wavelength of the frequency is closer to the size of the particles in the coating through which the vibration is travelling. This means that there is more scattering of the higher frequency waves, making them less reliable. More complicated mode shapes, such as those associated with higher frequencies, have more nodes and therefore require more perfect interaction between waves to create the standing wave pattern for that shape.

Figures 5.5 and 5.6 show the simulated and SLDV results for the cathodes with coating thicknesses of $38 \mu \mathrm{m}$ and $26 \mu \mathrm{m}$, respectively. Table 5.1 lists the values obtained for the Young's 


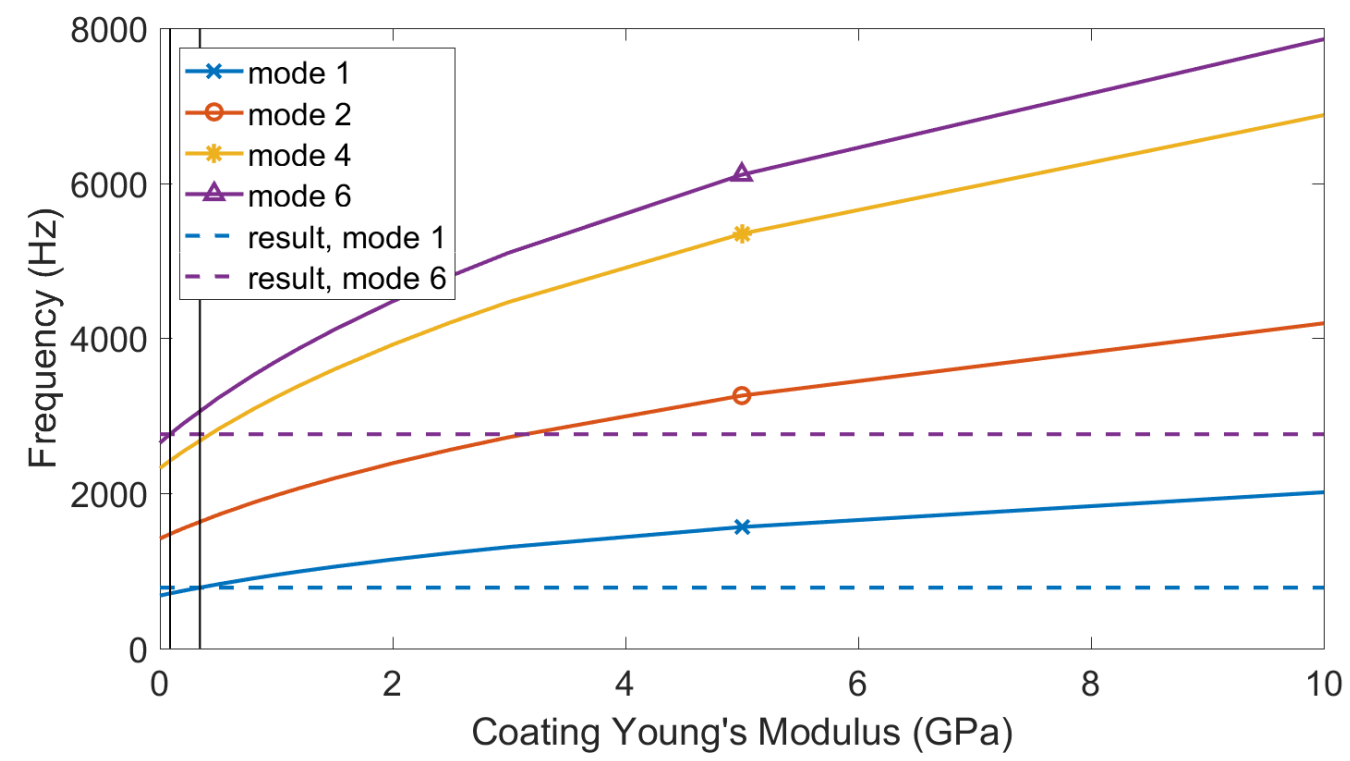

Figure 5.4: Simulation of battery cathode with properties listed under "Coating 1" in Table 5.1. For the simulation, the Young's modulus of the coating was varied to find the resonance frequencies. The dashed lines show the frequencies of the peaks for this film in our experimental results with the SLDV (modes 1 and 6), and the vertical lines show where the intersections occur between the simulation modes 1 and 6 and the SLDV results.

modulus of each cathode from comparing the simulations with the peaks from the SLDV results ( $E$, SLDV), as well as the values from comparing the simulations and the peaks seen in Fig. 4.3 ( $E$, peaks). The peaks for each method, though similar, were not exactly the same, resulting in some difference in the extracted Young's modulus for each film. This could be due to heterogeneity in the cathodes. A higher resolution for the FFT in the SLDV measurements, as well as narrower bandwidth frequency chirps, would also give more accurate results which may correlate better with the other tests.

The Young's modulus values obtained from the combination of SLDV results and the numerical model are higher than most of the values seen in the literature, which range from MPa to GPa. Forouzan et al. measured the elasticity of the coating (once delaminated from the current collector) and found it to be about 11.3 MPa [49], while Nadimpalli et al. used the rule of mixtures to calculate a cathode coating elasticity of $40 \mathrm{GPa}$ [54]. The rule of mixtures doesn't account for how materials actually interact, so the measured value is likely more reliable, but much lower than 


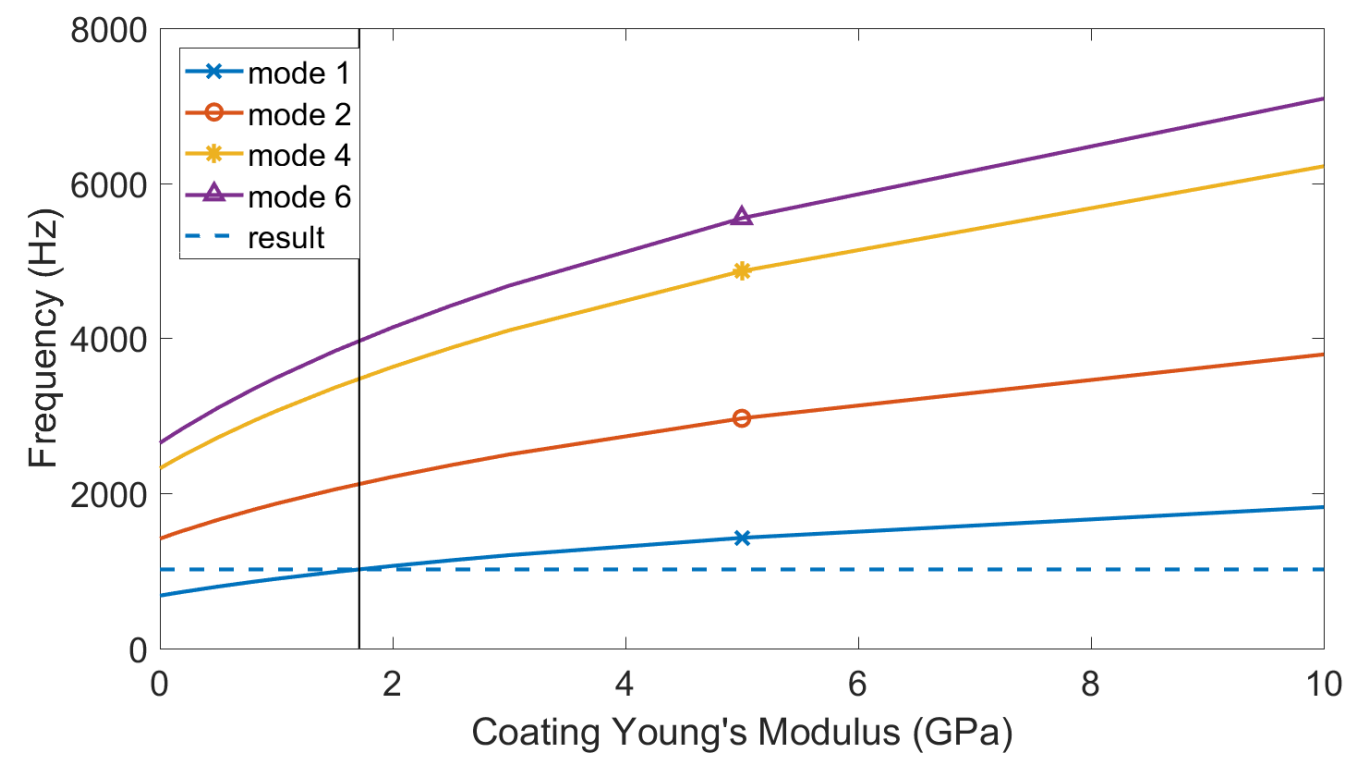

Figure 5.5: Simulation of battery cathode with properties listed under "Coating 2" in Table 5.1. For the simulation, the Young's modulus of the coating was varied to find the resonance frequencies. The dashed line shows the frequency of the peak for this film in our experimental results with the SLDV, and the vertical line shows the intersection between mode 1 of the simulation results and the SLDV result.

those obtained from our experiments. However, with such a large range for the elasticity of the battery coating, it is difficult to have a good benchmark for the Young's modulus values we found.

The simulations shown in Figs. 5.4-5.6 used assumed values for the properties of the aluminum current collector, but variations from these values could cause changes in the results. To check this, we ran simulations for the battery films with the coating elasticities extracted from the SLDV results with various values for the Young's modulus of the aluminum. Figures 5.7 and 5.8 show the results for the battery cathode with "Coating 1" in Table 5.1 with the two Young's modulus values extracted using modes 1 and 6, respectively. These figures show that the resonance frequencies for mode 1 are not changed much even by fairly large changes (10 GPa) in the Young's modulus of the current collector, but the values for mode 6 do change a little more noticeably. The same was true for the other two films, shown in Figs. 5.9 and 5.10. This indicates that the results for resonance frequency are not very sensitive to the assumed value of the Young's modulus for the current collector. However, the higher modes are slightly more sensitive to those changes. Although the results are not changed much by the uncertainty in the aluminum properties, this is 


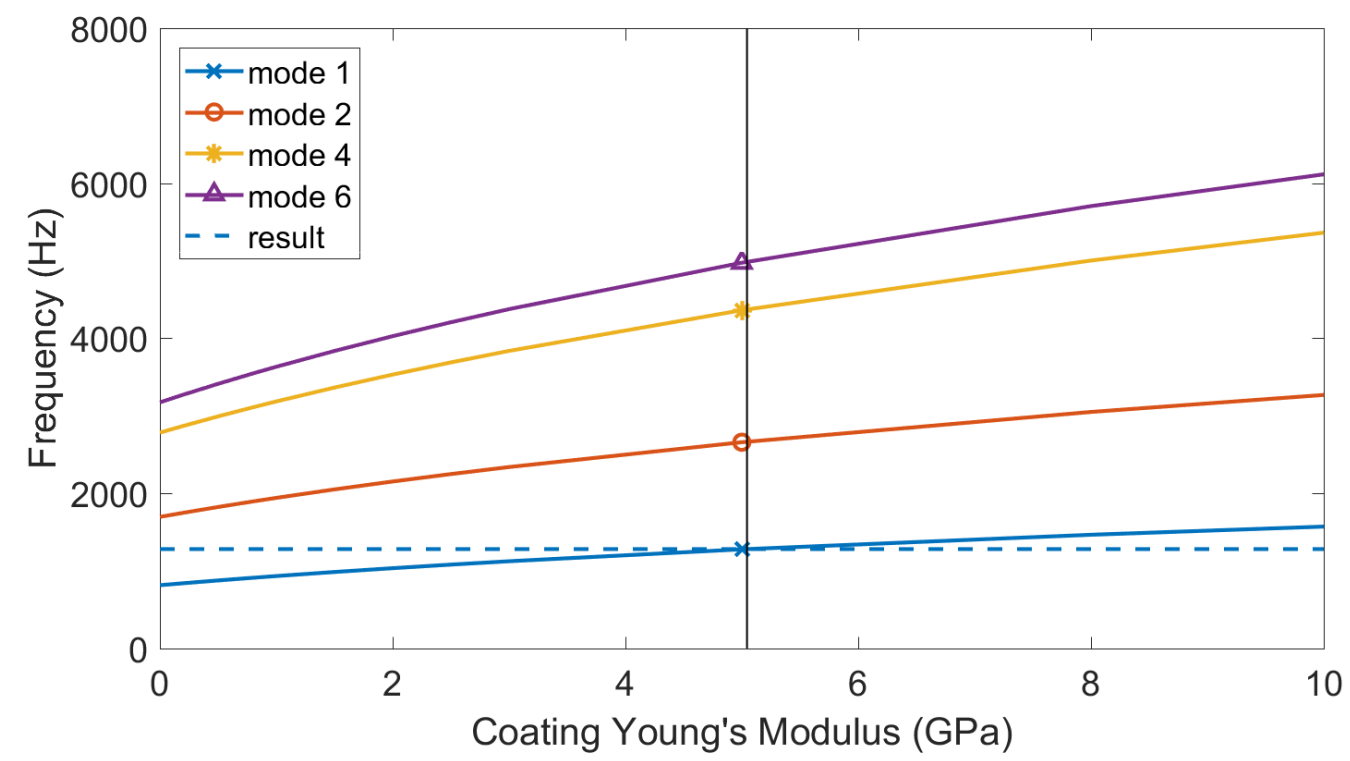

Figure 5.6: Simulation of battery cathode with properties listed under "Coating 3" in Table 5.1. For the simulation, the Young's modulus of the coating was varied to find the resonance frequencies. The dashed line shows the frequency of the peak for this film in our experimental results with the SLDV, and the vertical line shows the location of the intersection between mode 1 of the simulation results and the SLDV result.

another factor that could contribute to the difference between the Young's modulus values obtained from modes 1 and 6 for the film with a $42-\mu$ m-thick coating.

The changes made to our setup, as described in Chapter 3, improved the consistency of the experimental results. We were able to get similar results from repeated tests, but they were not always the same. Although the results were still not as repeatable as we hoped, they do show that we can use resonances to distinguish between films with different mechanical properties, to monitor drying state, and in combination with a numerical model to find the Young's modulus of the battery coating. 


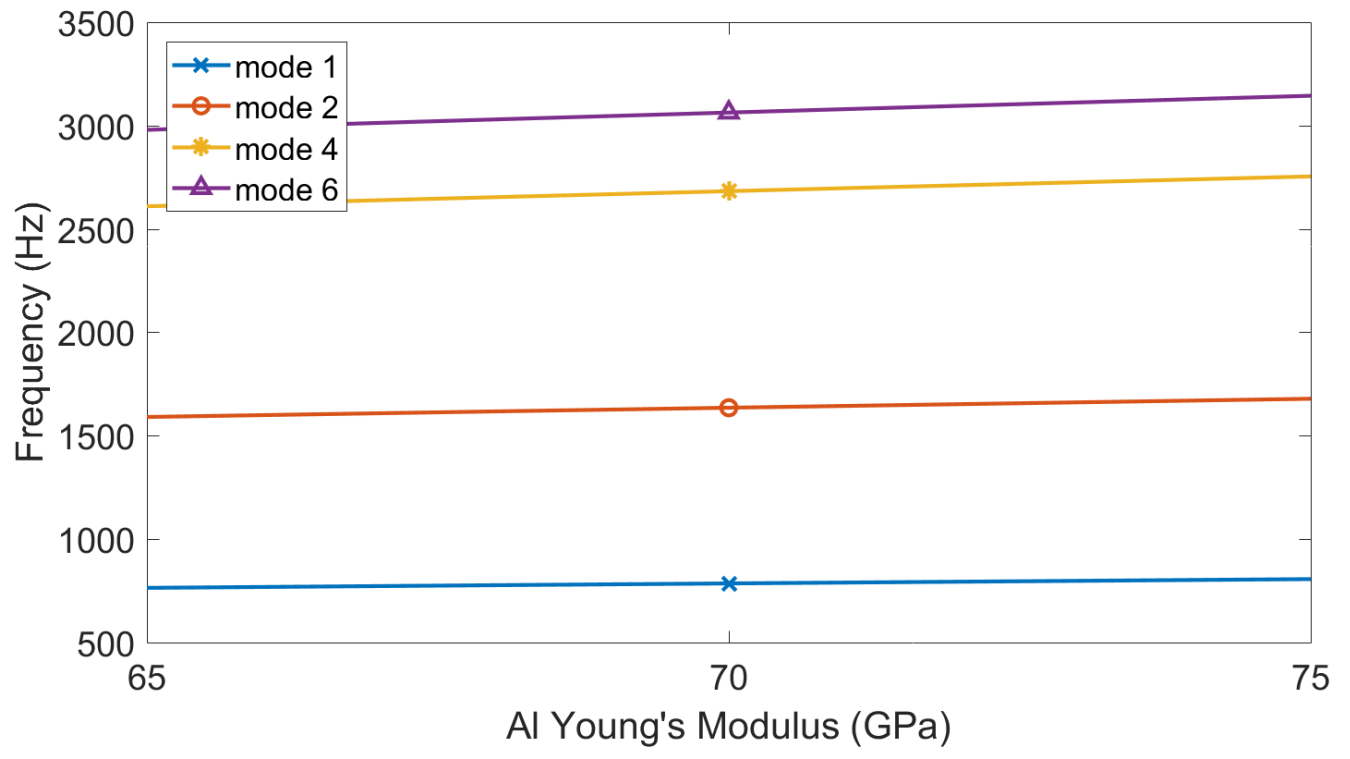

Figure 5.7: Simulation of battery cathode with properties listed under "Coating 1" in Table 5.1, using the Young's modulus value given by the SLDV results for mode 1 (344 MPa). For the simulation, the Young's modulus of the aluminum current collector was varied to find how sensitive the resonance frequencies are to changes in the assumed properties of the current collector.

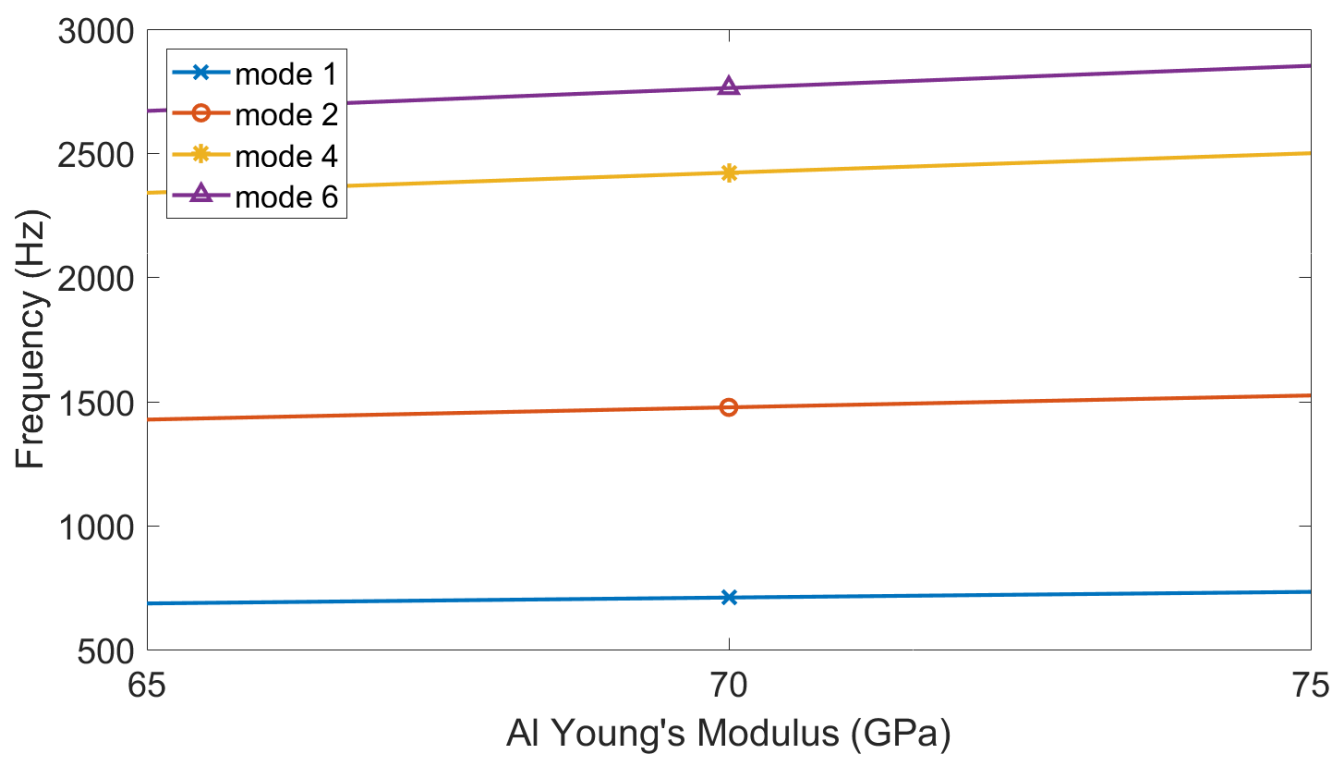

Figure 5.8: Simulation of battery cathode with properties listed under "Coating 1" in Table 5.1, but with a Young's modulus of $87 \mathrm{MPa}$, the value given by the SLDV results for mode 6 in Fig. 5.4. For the simulation, the Young's modulus of the aluminum current collector was varied to find how sensitive the resonance frequencies are to changes in the assumed properties of the current collector. 


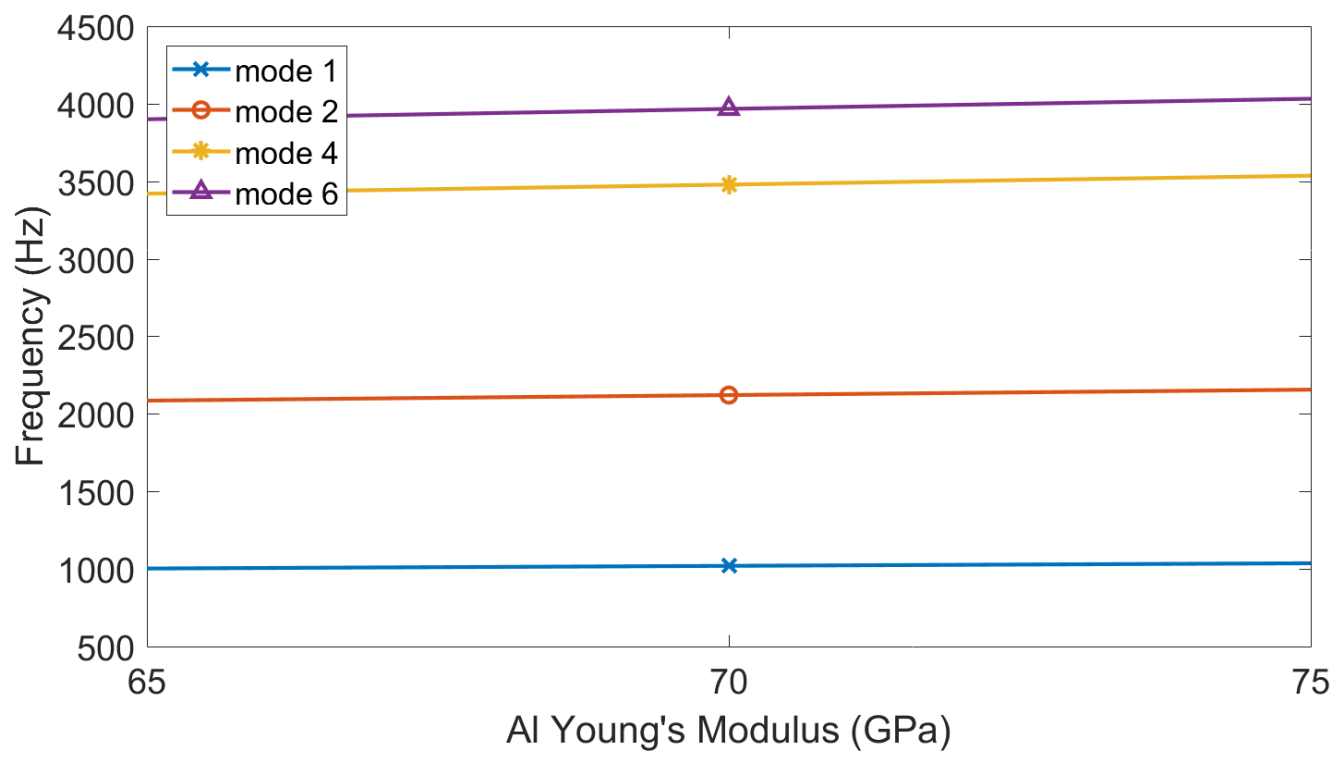

Figure 5.9: Simulation of battery cathode with properties listed under "Coating 2" in Table 5.1. For the simulation, the Young's modulus of the aluminum current collector was varied to find how sensitive the resonance frequencies are to changes in the assumed properties of the current collector.

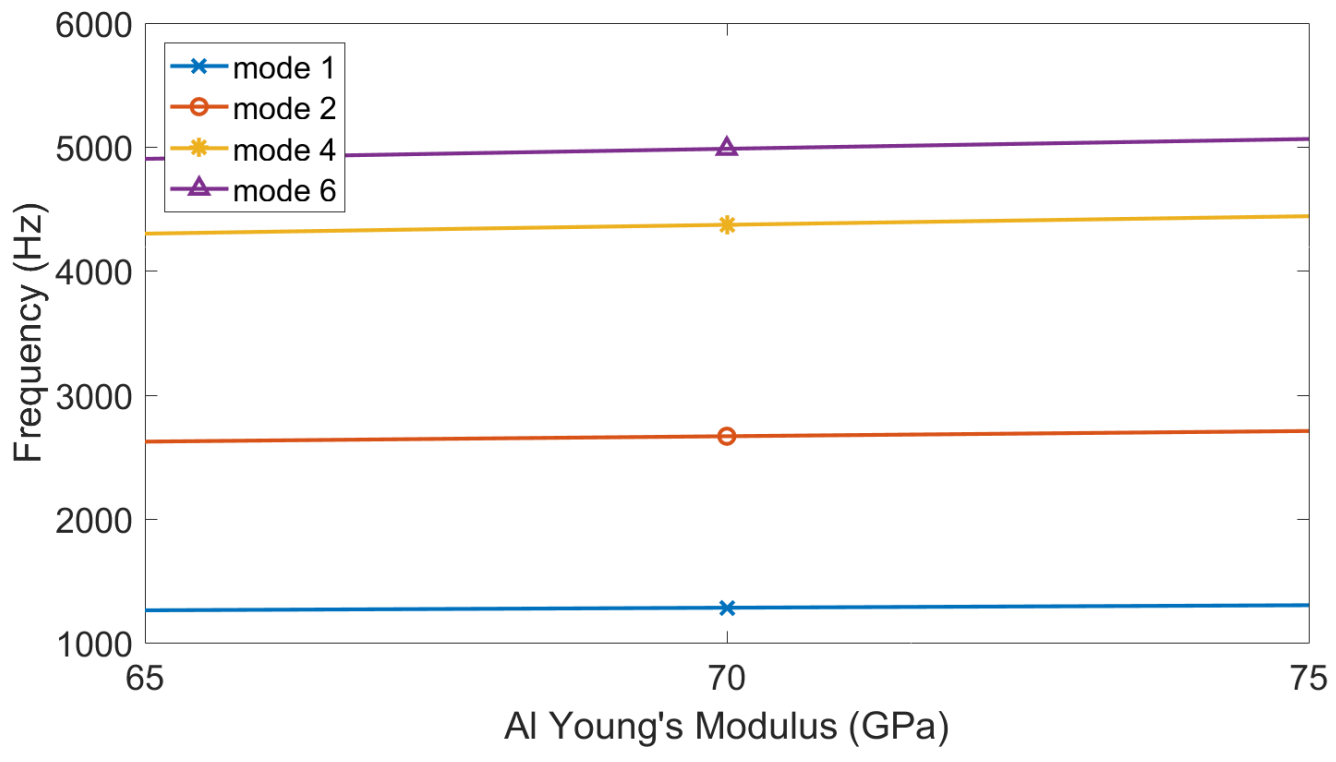

Figure 5.10: Simulation of battery cathode with properties listed under "Coating 3" in Table 5.1. For the simulation, the Young's modulus of the aluminum current collector was varied to find how sensitive the resonance frequencies are to changes in the assumed properties of the current collector. 


\section{CHAPTER 6. CONCLUSION}

\subsection{Summary}

This thesis described my work using acoustic resonance measurements of battery electrodes to differentiate between films with different mechanical characteristics, specifically density, thickness, and elasticity. The equations in Chapter 2 show how the frequencies at which thin plates and membranes vibrate change based on each of these properties. Simulations done using ANSYS software show that, although neither thin plate nor membrane theory fully describe the behavior of the two-layer battery films, overall the electrodes follow the trend of membrane theory.

We used a pulsed infrared laser or a speaker to excite resonances in commercial battery cathodes and were able to distinguish between films with different coating thicknesses, as our simulations predicted would be possible. Measurements made with a scanning laser Doppler vibrometer confirmed that the frequencies we were examining were the fundamental modes of the films. This allowed us to combine the experimental results with simulations to find values for the Young's modulus of the battery coatings. We used the same excitation setup to take measurements of an aluminum film with drying paint and found that the resonance frequency shifted as the paint dried, indicating that resonances could be used to monitor a drying process.

\subsection{Future Work}

Since this research is still a fairly new project, there is a lot work to be done. One of the most important problems to solve is that of clamping and repeatable results. Clamping has consistently been an issue with repeatability, makes the setup less conducive to a roll-to-roll manufacturing process, and can cause undesirable calendering of the battery films. One potential solution to this problem is scanning for zero-group-velocity (ZGV) modes, a method briefly described in Chapter 1. This would allow for an unconstrained vibration area but still enable local measure- 
ments of the material characteristics. However, this method is not without its own challenges. The $\mathrm{ZGV}$ resonances occur at much higher frequencies than the resonances examined in this thesis, so different methods of excitation and especially detection would be necessary for this type of measurement. Because battery electrode films are so thin, the ZGV resonances may even be in the gigahertz range, which would be difficult to measure.

A better method of detection should be investigated, particularly if switching to looking for ZGV modes. It should be determined if some kind of laser displacement sensor would work for this application, or looking at the light scattering off the rough surface of the battery coating. The dark coating on the battery films may not be reflective enough for this measurement to work well, but if it did work, the measurements would be more accurate and less sensitive to noise. For battery films with only one side coated with cathode or anode material, such as those examined in the experiments in this thesis, a laser displacement sensor should work if directed at the reflective metal side of the film.

A different excitation source would be ideal. While the laser was useful in that it coupled large amounts of energy to the films without causing acoustic noise the microphone would detect, safety is a concern and causes inconvenience. The laser may not be a good option for a manufacturing environment, where implementing the proper safety protocols for such a high-energy infrared laser could be problematic. Furthermore, the laser has to be attenuated by different amounts for each type of film to avoid ablation of the coating. Being able to avoid these problems altogether would be both safer and convenient. The results shown in this thesis using acoustic excitation, both with the SLDV and the microphone detection, show that adequate excitation is possible without the laser, and that for the frequencies we examine here, acoustic excitation works well.

More films need to be tested, particularly films with only one varying parameter. The commercial-grade films tested here had different coating materials, thicknesses, and densities, so we could not separate the effects of each parameter on the resonance frequency. Another potentially interesting aspect is examining both the width of the resonance peaks and the distance between peaks corresponding to different modes. This may be useful in determining the effect of each material parameter. Independent verification of the parameters is also needed, especially for the Young's modulus calculated using the combination of simulations and experimental results. 
Since there are methods currently used to measure the mechanical properties, this is definitely possible.

The end goal of this project is to create a probe that can be used in a roll-to-roll process to quantify the heterogeneity of the mechanical properties of battery electrode films. Once this probe is made, it will be necessary to determine what resolution is needed in these measurements to decide if a film is sufficiently homogeneous. The probe should also be used to correlate heterogeneity in the mechanical properties to variations in the electrical and ionic conductivities.

\subsection{Conclusion}

Battery safety and quality could be improved by reducing heterogeneities in battery electrode films. Measuring acoustic resonances of these films and looking for shifts in the resonance is one way to monitor changes in the mechanical properties. Acoustic response could also be used to track the state of drying, as we were able to detect changes in mass and stiffness during a drying process. Additionally, the combination of resonance measurements and a numerical model can enable the determination of the Young's modulus of the battery film, which is difficult to measure. Although more work is needed in this area, we are hopeful that acoustic resonance measurements could be a form of fast, non-destructive quality control in battery manufacturing. 


\section{REFERENCES}

[1] A. Fotouhi, D. J. Auger, K. Propp, S. Longo, and M. Wild, "A review on electric vehicle battery modelling: From lithium-ion toward lithium-sulphur," Renewable and Sustainable Energy Reviews, vol. 56, pp. 1008-1021, 2016. 1

[2] IEA, "Global EV outlook 2017," International Energy Agency, Paris, France, Tech. Rep., 2017. 1

[3] B. Scrosati and J. Garche, "Lithium batteries: Status, prospects and future," Journal of Power Sources, vol. 195, no. 9, pp. 2419-2430, 2010. 1

[4] G. Chen and T. J. Richardson, "Continuity and performance in composite electrodes," Journal of Power Sources, vol. 195, no. 16, pp. 5387-5390, 2010. 1, 4

[5] S. J. Harris and P. Lu, "Effects of inhomogeneities—nanoscale to mesoscale—on the durability of Li-ion batteries," The Journal of Physical Chemistry C, vol. 117, no. 13, pp. 6481-6492, February 2013. 1, 5

[6] K. Higa, H. Zhao, D. Y. Parkinson, H. Barnard, M. Ling, G. Liu, and V. Srinivasan, "Electrode slurry particle density mapping using X-ray radiography," Journal of the Electrochemical Society, vol. 164, no. 2, pp. A380-A388, 2017. 1, 5

[7] J. Lee, S. Sung, Y. Kim, J. D. Park, and K. H. Ahn, "A new paradigm of materials processing - heterogeneity control," Current Opinion in Chemical Engineering, vol. 16, pp. 16-22, 2017. 1, 5

[8] B. J. Lanterman, A. A. Riet, N. S. Gates, J. D. Flygare, A. D. Cutler, J. E. Vogel, D. R. Wheeler, and B. A. Mazzeo, "Micro-four-line probe to measure electronic conductivity and contact resistance of thin-film battery electrodes," Journal of the Electrochemical Society, vol. 162, no. 10, pp. A2145-A2151, 2015. 2, 4

[9] J. Tarascon and M. Armand, "Issues and challenges facing rechargeable lithium batteries," Nature, vol. 414, pp. 359-367, 2001. 3, 4

[10] J. B. Goodenough, General Concepts. Wiley-VCH Verlag GmbH, 2007, pp. 1-25. 3

[11] K. Tagawa and R. J. Brodd, Production Processes for Fabrication of Lithium-Ion Batteries. New York, NY: Springer New York, 2009, ch. 8, pp. 181-194. 3, 4, 48, 51

[12] L. Lu, X. Han, J. Li, J. Hua, and M. Ouyang, "A review on the key issues for lithium-ion battery management in electric vehicles," Journal of Power Sources, vol. 226, pp. 272-288, 2013. 4

[13] K. Zeng and J. Zhu, "Surface morphology, elastic modulus and hardness of thin film cathodes for Li-ion rechargeable batteries," Mechanics of Materials, vol. 91, no. 2, pp. 323-332, December 2015. 5 
[14] G. Chow, P. Miller, and J. Wang, "Correlation between laser-induced surface acoustic waves and nanoindentation on elastic modulus measurement of a nanoporous zeolite thin film," Experimental Mechanics, vol. 55, no. 3, pp. 647-650, March 2015. 5

[15] S. S. Kumar, A. Fartash, M. Grimsditch, I. K. Schuller, and R. S. Kumar, "Elastic properties of polymer films. 1. Elastic constants of a polyimide film determined by Brillouin scattering and mechanical techniques," Macromolecules, vol. 26, no. 23, pp. 6184-6189, 1993. 5

[16] A. G. Every, "Measurement of the near-surface elastic properties of solids and thin supported films," Measurement Science and Technology, vol. 13, no. 5, p. R21, 2002. 5

[17] B. A. Mazzeo, A. N. Patil, R. C. Hurd, J. M. Klis, T. T. Truscott, and W. S. Guthrie, "Aircoupled impact-echo delamination detection in concrete using spheres of ice for excitation," Journal of Nondestructive Evaluation, vol. 33, no. 3, pp. 317-326, Sep 2014. 6

[18] B. E. Anderson, L. Pieczonka, M. C. Remillieux, T. J. Ulrich, and P. L. Bas, "Stress corrosion crack depth investigation using the time reversed elastic nonlinearity diagnostic," The Journal of the Acoustical Society of America, vol. 141, no. 1, pp. EL76-EL81, 2017. 6

[19] M. Silva, R. Gouyon, and F. Lepoutre, "Hidden corrosion detection in aircraft aluminum structures using laser ultrasonics and wavelet transform signal analysis," Ultrasonics, vol. 41, no. 4, pp. 301-305, 2003. 6, 7

[20] D. Schneider and M. Tucker, "Non-destructive characterization and evaluation of thin films by laser-induced ultrasonic surface waves," Thin Solid Films, vol. 290, pp. 305-311, 1996. 6

[21] A. Neubrand and P. Hess, "Laser generation and detection of surface acoustic waves: Elastic properties of surface layers," Journal of Applied Physics, vol. 71, no. 1, pp. 227-238, 1992. 6

[22] D. Hurley, V. Tewary, and A. Richards, "Thin-film elastic-property measurements with laserultrasonic SAW spectrometry," Thin Solid Films, vol. 398, pp. 326-330, 2001. 6

[23] J. Sermeus, R. Sinha, K. Vanstreels, P. M. Vereecken, and C. Glorieux, "Determination of elastic properties of a $\mathrm{MnO} 2$ coating by surface acoustic wave velocity dispersion analysis," Journal of Applied Physics, vol. 116, no. 2, p. 023503, 2014. 6

[24] Q. Zhang, X. Xiao, Y. Cheng, and M. W. Verbrugge, "A non-destructive method for measuring the mechanical properties of ultrathin films prepared by atomic layer deposition," Applied Physics Letters, vol. 105, no. 6, p. 061901, August 2014. 6

[25] S. Kitazawa, A. Chiba, and E. Wakai, "Laser-induced surface acoustic waves and their detection via diagnostic systems for detecting radiation damage on steel materials of nuclear devices," Nuclear Instruments and Methods in Physics Research Section A: Accelerators, Spectrometers, Detectors and Associated Equipment, vol. 786, pp. 47-50, June 2015. 6

[26] T. Grabec, P. Sedlák, P. Stoklasová, M. Thomasová, D. Shilo, M. Kabla, H. Seiner, and M. Landa, "In situ characterization of local elastic properties of thin shape memory films by surface acoustic waves," Smart Materials and Structures, vol. 25, no. 12, p. 127002, December 2016. 6 
[27] R. Côte, T. V. der Donck, J. Celis, and C. Glorieux, "Surface acoustic wave characterization of a thin, rough polymer film," Thin Solid Films, vol. 517, no. 8, pp. 2697-2701, February 2009. 6

[28] G. Chow, E. Uchaker, G. Cao, and J. Wang, "Laser-induced surface acoustic waves: An alternative method to nanoindentation for the mechanical characterization of porous nanostructured thin film electrode media," Mechanics of Materials, vol. 91, no. 2, pp. 333-342, December 2015. 6

[29] W. Gao, C. Glorieux, and J. Thoen, "Laser ultrasonic study of Lamb waves: determination of the thickness and velocities of a thin plate," International Journal of Engineering Science, vol. 41, no. 2, pp. 219-228, 2003. 7, 28

[30] S. E. Bobbin, J. W. Wagner, and R. C. Cammarata, "Determination of the flexural modulus of thin films from measurement of the first arrival of the symmetric Lamb wave," Applied Physics Letters, vol. 59, no. 13, pp. 1544-1546, 1991. 7, 28

[31] S. D. Holland and D. E. Chimenti, "Air-coupled acoustic imaging with zero-group-velocity Lamb modes," Applied Physics Letters, vol. 83, no. 13, pp. 2704-2706, 2003. 7

[32] C. M. Grünsteidl, I. A. Veres, and T. W. Murray, "Experimental and numerical study of the excitability of zero group velocity Lamb waves by laser-ultrasound," The Journal of the Acoustical Society of America, vol. 138, no. 1, pp. 242-250, 2015. 7

[33] C. Prada, O. Balogun, and T. W. Murray, "Laser-based ultrasonic generation and detection of zero-group velocity Lamb waves in thin plates," Applied Physics Letters, vol. 87, no. 19, p. 194109, 2005. 7

[34] D. Clorennec, C. Prada, and D. Royer, "Laser ultrasonic inspection of plates using zero-group velocity Lamb modes," IEEE Transactions on Ultrasonics, Ferroelectrics, and Frequency Control, vol. 57, no. 5, pp. 1125-1132, May 2010. 7

[35] C. Grünsteidl, T. W. Murray, T. Berer, and I. A. Veres, "Inverse characterization of plates using zero group velocity Lamb modes," Ultrasonics, vol. 65, pp. 1-4, 2016. 7

[36] A. Fartash, "Young's modulus of a thin film determined from the peak shifts of its membrane modes," Applied Physics Letters, vol. 61, no. 17, pp. 2024-2026, 1992. 7, 14

[37] A. Biswas, T. Weller, and L. P. B. Katehi, "Stress determination of micromembranes using laser vibrometry," Review of Scientific Instruments, vol. 67, no. 5, pp. 1965-1969, 1996.7

[38] C. Malhaire, "Comparison of two experimental methods for the mechanical characterization of thin or thick films from the study of micromachined circular diaphragms," Review of Scientific Instruments, vol. 83, no. 5, p. 055008, 2012. 7

[39] F. Gamboa, A. López, F. Avilés, J. E. Corona, and A. I. Oliva, "A vibrating reed apparatus to measure the natural frequency of multilayered thin films," Measurement Science and Technology, vol. 27, no. 4, p. 045002, Apr. 2016. 7 
[40] R. D. Costley, M. E. Henderson, H. Patel, E. W. Jones, G. M. Boudreaux, and M. J. Plodinec, "The measurement of pressure and level of fill in sealed storage drums," NDT \& E International, vol. 40, no. 4, pp. 300-308, 2007. 8, 52

[41] A. G. Hsieh, S. Bhadra, B. J. Hertzberg, P. J. Gjeltema, A. Goy, J. W. Fleischer, and D. A. Steingart, "Electrochemical-acoustic time of flight: in operando correlation of physical dynamics with battery charge and health," Energy \& Environmental Science, vol. 8, pp. 1569$1577,2015.8$

[42] L. Kinsler, A. Frey, A. Coppens, and J. Sanders, Fundamentals of Acoustics, 4th ed. New York, New York: Wiley-VCH, 1999. 9, 11, 15, 47, 52

[43] S. Timoshenko, D. H. Young, and W. Weaver, Jr., Vibration Problems in Engineering, 4th ed. New York, New York: John Wiley \& Sons, Inc., 1974. 9, 10, 11, 12, 13

[44] E. Ruggiero, "Modeling and control of SPIDER satellite components," Ph.D. dissertation, Virginia Polytechnic Institute and State University, Blacksburg, VA, 2005. 10

[45] A. Fartash, I. K. Schuller, and M. Grimsditch, "Thin-film modeling for mechanical measurements: Should membranes be used or plates?" Journal of Applied Physics, vol. 71, no. 9, pp. 4244-4248, 1992. 10

[46] N. H. Fletcher, Acoustic Systems in Biology. New York, New York: Oxford University Press, 1992. 11

[47] L. Dong, M. Grissom, and F. T. Fisher, "Resonant frequency of mass-loaded membranes for vibration energy harvesting applications," AIMS Energy, vol. 3, no. 3, pp. 344-359, August 2015. 11

[48] A. W. Leissa, "Vibration of plates," Ohio State Univ Colombus, Tech. Rep., 1969. 12

[49] M. M. Forouzan, C. Chao, D. Bustamante, B. A. Mazzeo, and D. R. Wheeler, "Experiment and simulation of the fabrication process of lithium-ion battery cathodes for determining microstructure and mechanical properties," Journal of Power Sources, vol. 312, pp. 172-183, 2016. 15,53

[50] R. A. Serway, J. S. Faughn, and C. Vuille, College Physics, 8th ed. Cengage Learning, 2008, vol. 10. 15

[51] B. Liu, X. Feng, and S. Zhang, "The effective Young's modulus of composites beyond the Voigt estimation due to the Poisson effect," Composites Science and Technology, vol. 69, no. 13, pp. 2198-2204, 2009. 15, 16

[52] J. Hatch, Aluminum: Properties and Physical Metallurgy. Metals Park, OH, USA: ASM International, 1984, ch. 1, pp. 13-14. 28

[53] B. M. Watrasiewicz and M. J. Rudd, Laser Doppler Measurements. London, England: Butterworths, 1976. 29

[54] S. P. Nadimpalli, V. A. Sethuraman, D. P. Abraham, A. F. Bower, and P. R. Guduru, "Stress evolution in lithium-ion composite electrodes during electrochemical cycling and resulting 
internal pressures on the cell casing," Journal of the Electrochemical Society, vol. 162, no. 14, pp. A2656-A2663, 2015. 53 


\section{APPENDIX A. ADDITIONAL INFORMATION}

\section{A.1 MATLAB Code}

Below is the MATLAB code used to obtain the frequency responses for the films. Listing A.1 contains the code used on the data obtained from frequency sweeps recorded by the PCB free-field microphone and NI module. The code has the user select the ".lvm" file or files generated from these devices, reads in the files, calculates the relative power spectral response, and plots this response. This code also requires the file "peakfinder.m", which can be downloaded from the MathWorks website.

Listing A.1: Code for graphing frequency response from frequency sweeps done with the PCB free-field microphone and NI module.

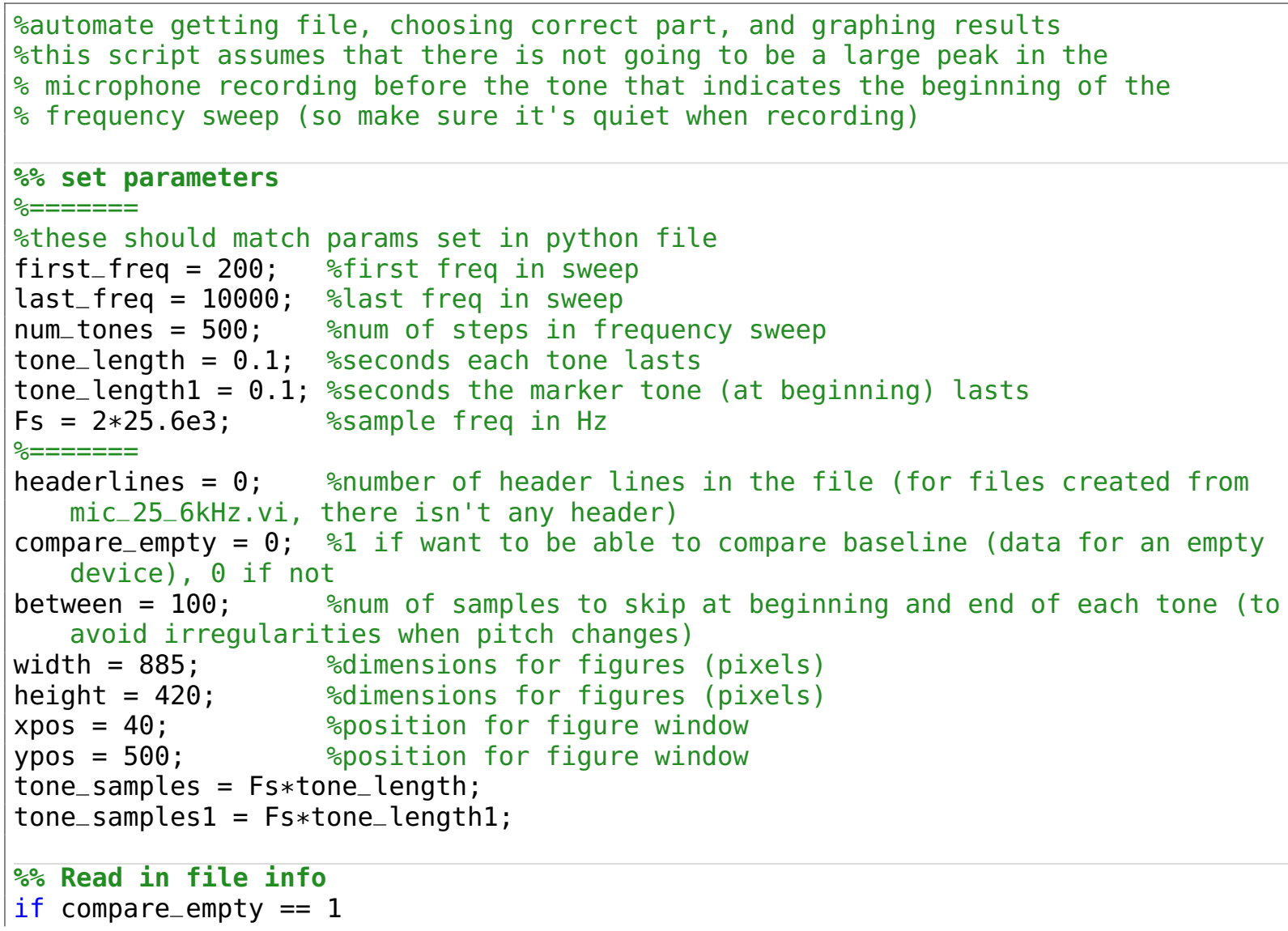




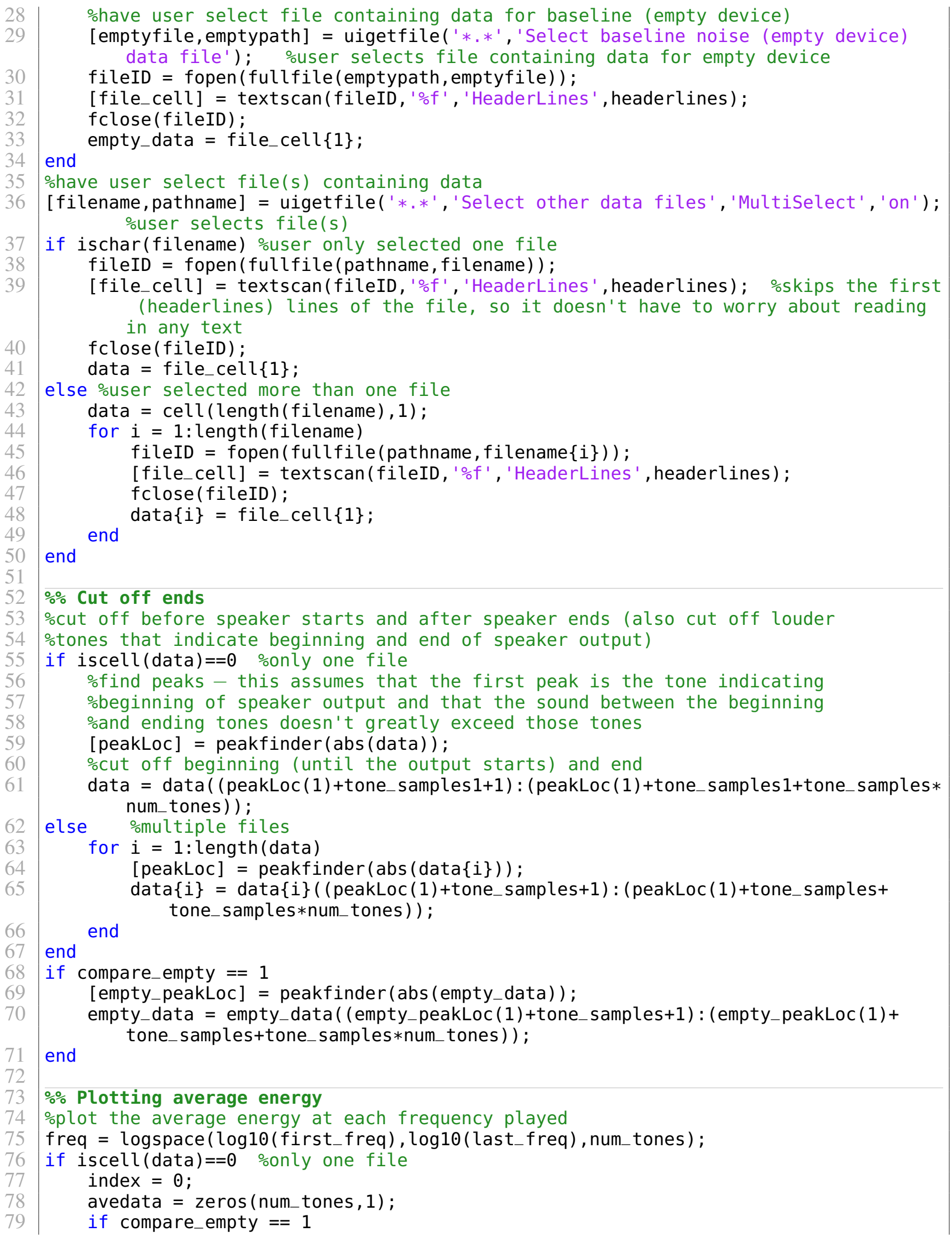




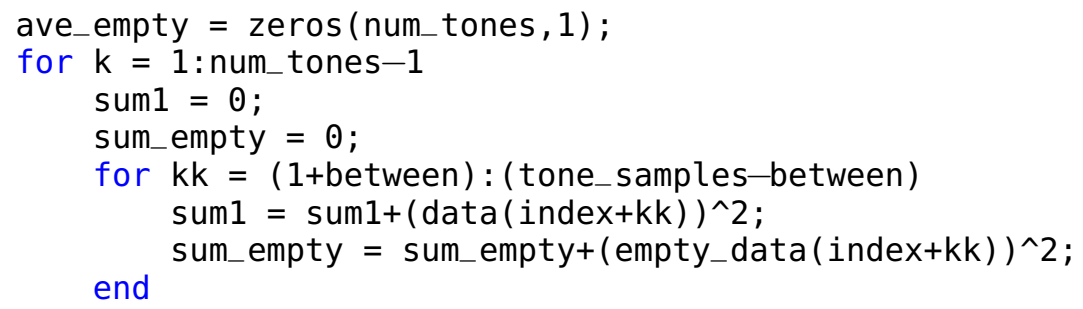



end

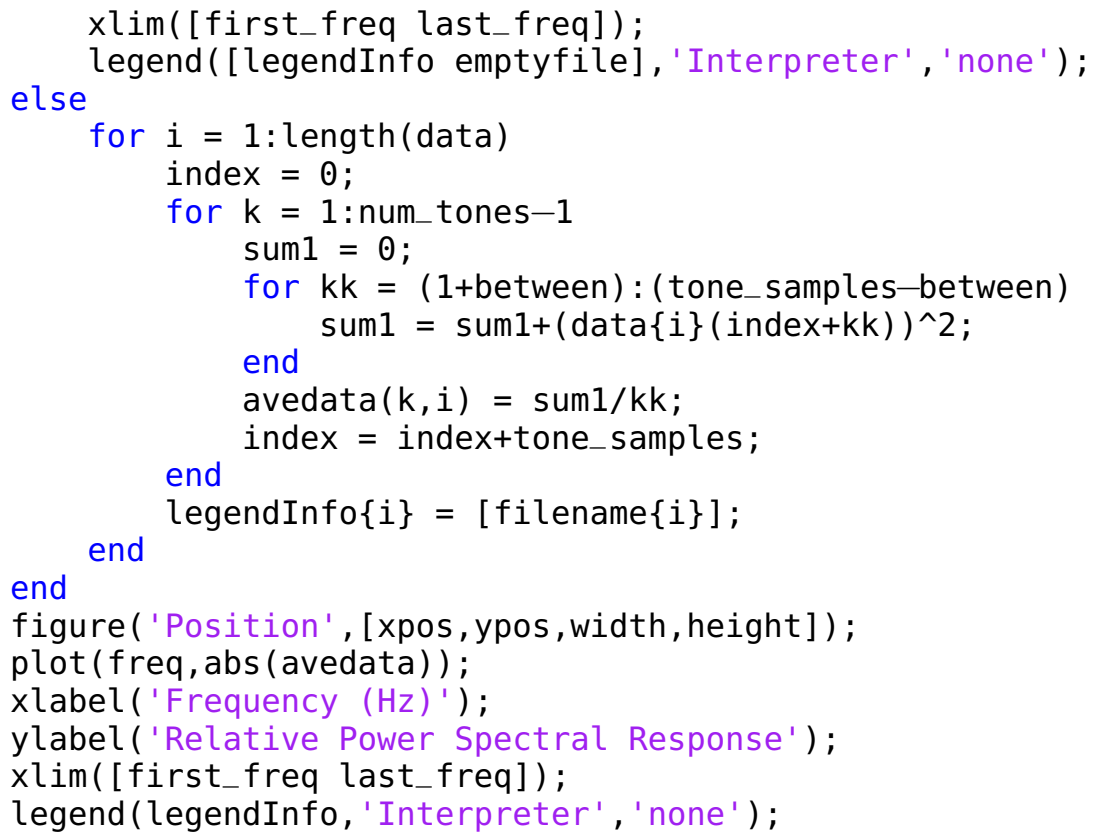

Listing A.2 shows the code used on the data recorded by the electret microphone. The code has the user select sets of ".txt" files generated from the Analog Discovery, reads in the files, finds the average of each set of files, calculates the FFT of the average, and plots the resulting frequency response. This code also attempts to fit a Gaussian to the peaks in the frequency response in order to obtain values for the resonance frequency. The file "peakfit.m", which can be downloaded from the MathWorks website, is required to run this code.

Listing A.2: Code for graphing frequency response and fitting Gaussian to the peaks from electret microphone data.

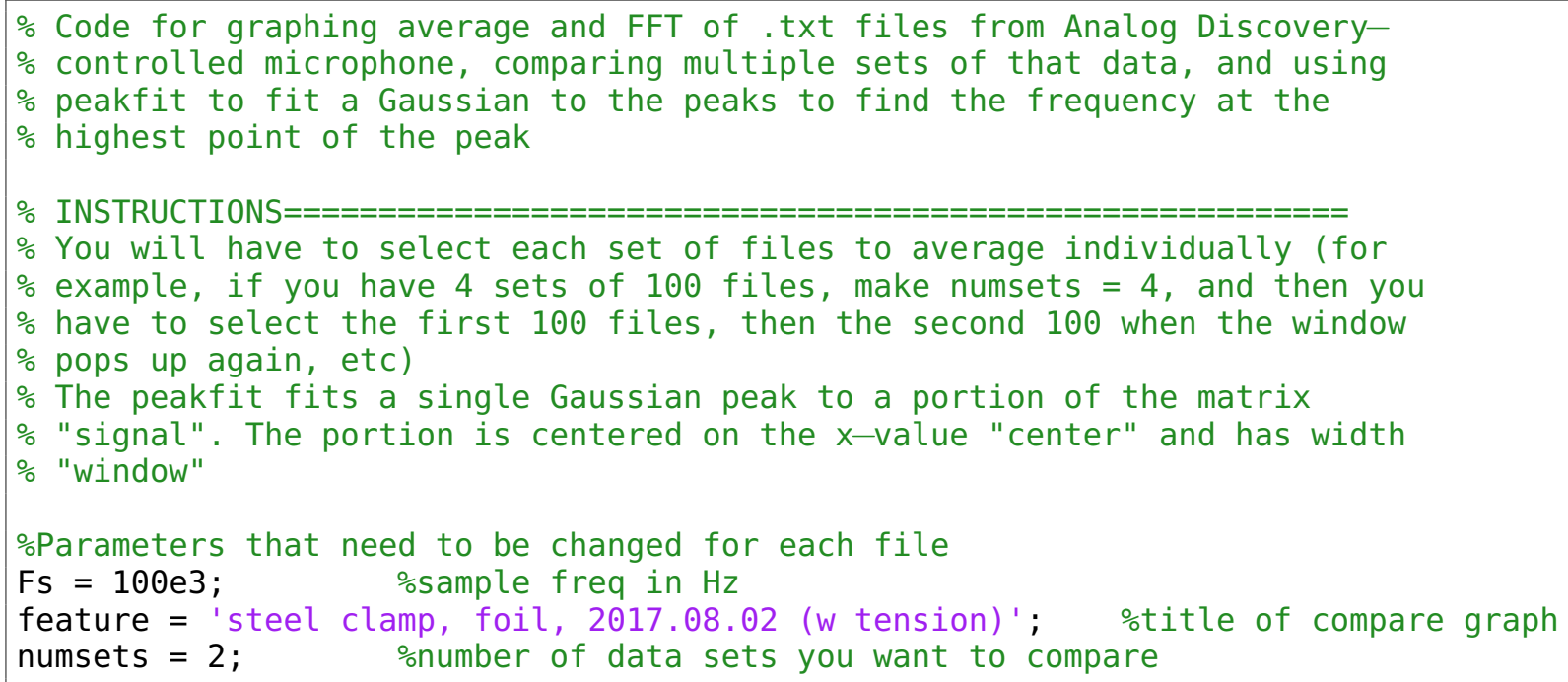




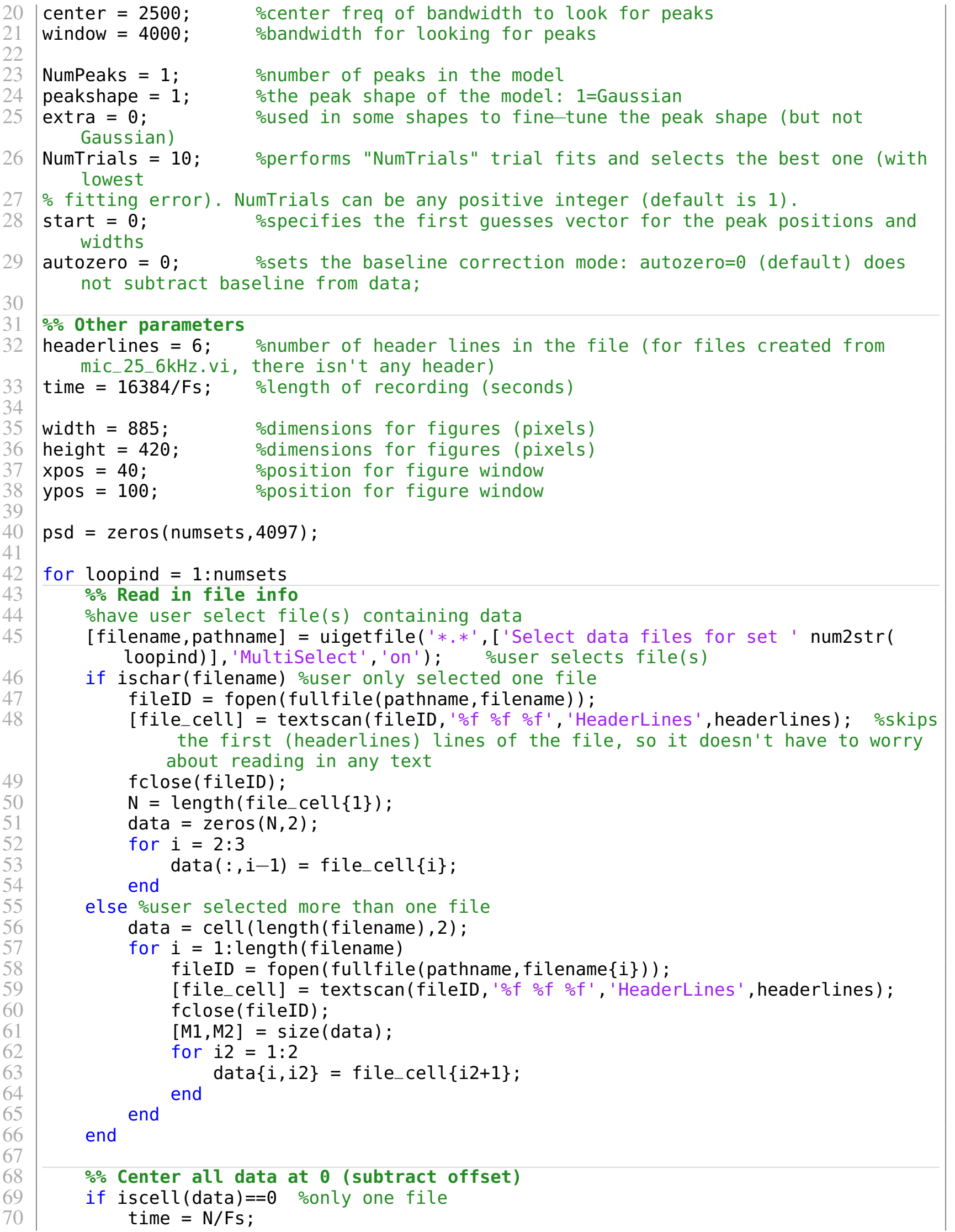




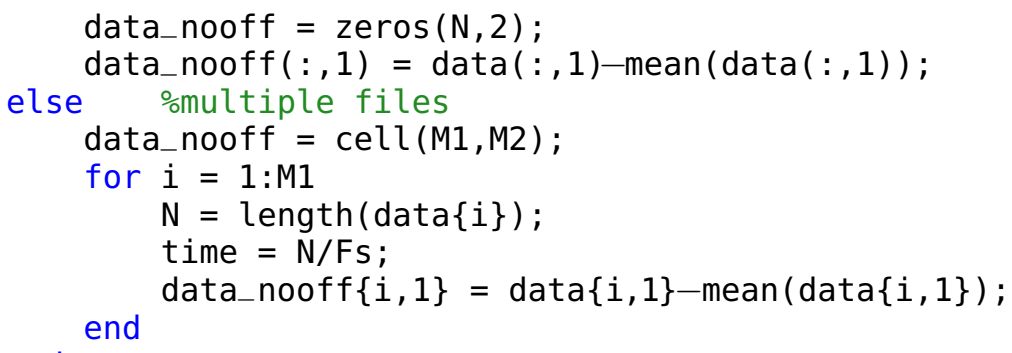




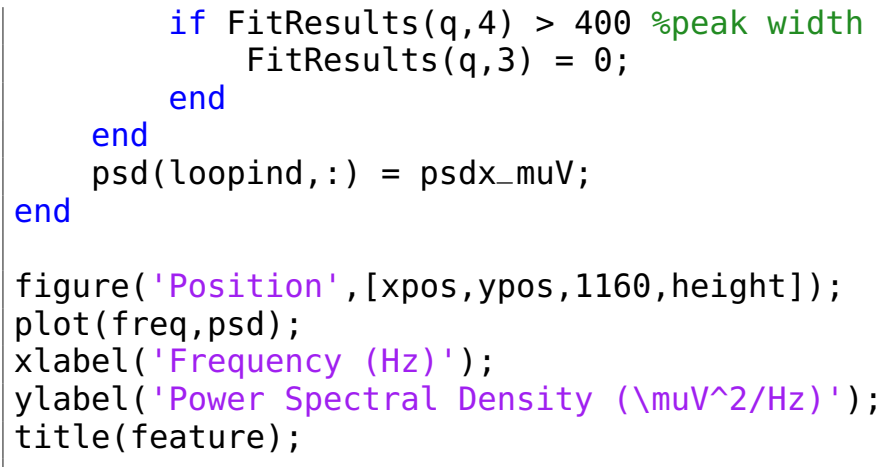

\section{A.2 Analog Discovery Settings}

As mentioned in Chapter 3, an Analog Discovery (AD) was used in the experimental setup as an oscilloscope to collect the output from the electret microphone, a voltage source for the electret microphone, and a wave generator to trigger the pulsed infrared laser. The oscilloscope was triggered by the wave generator and the sampling rate was set to $100 \mathrm{kHz}$. The logging was setup so that it saved a file of each triggered acquisition, up to either 200 or 1000 files. The power supply has an on/off for a $+5 \mathrm{~V}$ supply and a $-5 \mathrm{~V}$ supply, but the microphone requires a $2 \mathrm{~V}$ supply, so a voltage divider circuit was connected to the microphone. A diagram showing the full microphone circuit can be seen in Fig. A.1, where the output is connected to the AD oscilloscope channel. For the wave generator, the type of pulse was a square wave with $1 \mathrm{MHz}$ frequency, -1.5 $\mathrm{V}$ amplitude, $1.5 \mathrm{~V}$ offset (for a wave from 0 to $3 \mathrm{~V}$, as the laser required), $50 \%$ symmetry, and $0^{\circ}$ phase. It ran for $1 \mu \mathrm{s}$ and waited for $100 \mathrm{~ms}$ between pulses. 


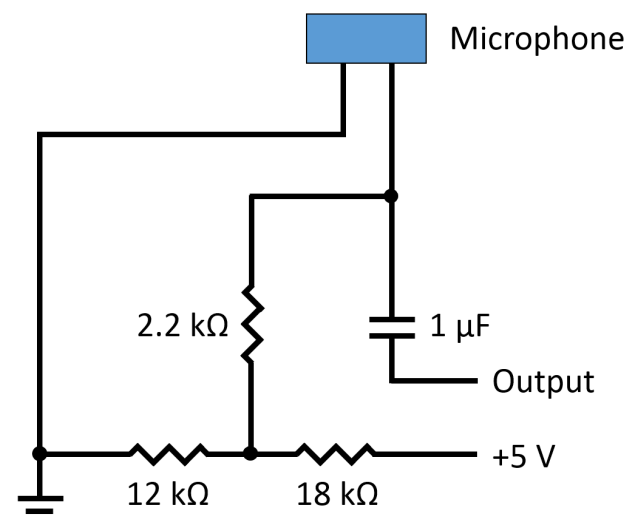

Figure A.1: Diagram showing the circuit used for the electret microphone. The microphone requires the capacitor and the $2.2 \mathrm{k} \Omega$ resistor, while the other resistors are part of a voltage divider to bring the Analog Discovery $5 \mathrm{~V}$ power supply down to the needed voltage of $2 \mathrm{~V}$. The output is connected to the AD oscilloscope channel. 